\title{
Extremely correlated quantum liquids
}

\begin{abstract}
B. Sriram Shastry
Physics Department, University of California, Santa Cruz, California 95064, USA

(Received 29 October 2009; revised manuscript received 11 December 2009; published 20 January 2010)

Extreme correlations arise as the limit of strong correlations, when the local interaction constant $U$ goes to infinity. This singular limit transforms canonical fermions to noncanonical Hubbard-type operators with a specific graded Lie algebra replacing the standard anticommutators. We are forced to deal with a fundamentally different and more complex lattice field theory. We study the $t-J$ model, embodying such extreme correlations. We formulate the picture of an extremely correlated electron liquid, generalizing the standard Fermi liquid. This quantum liquid breaks no symmetries, and has specific signatures in various physical properties, such as the Fermi-surface volume and the narrowing of electronic bands by spin- and density-correlation functions. We use Schwinger's source field idea to generate equations for the Green's function for the Hubbard operators. A local (matrix) scale transformation in the time domain to a quasiparticle Green's function is found to be optimal. This transformation allows us to generate vertex functions that are guaranteed to reduce to the bare values for high frequencies, i.e., are "asymptotically free." The quasiparticles are fractionally charged objects, and we find an exact Schwinger-Dyson equation for their Green's function, i.e., the self-energy is given explicitly in terms of the singlet and triplet particle-hole vertex functions. We find a hierarchy of equations for the vertex functions, and further we obtain Ward identities so that systematic approximations are feasible. An expansion in terms of the density of holes measured from the Mott Hubbard insulating state follows from the nature of the theory. A systematic presentation of the formalism is followed by some preliminary explicit calculations. We find a $d$-wave superconducting instability at low $T$ that formally resembles that found in the resonating valence-bond theory, but with a much reduced $T_{c}$.
\end{abstract}

DOI: 10.1103/PhysRevB.81.045121

PACS number(s): 71.10.Fd

\section{INTRODUCTION}

We present a theory of an extremely correlated quantum liquid (ECQL) in this paper. We believe it to be both necessary and useful to make a distinction between the class of systems studied here, and the more commonly addressed strongly correlated electron systems. The latter deals with models such as the Hubbard model for transition metals or copper oxide systems, and Kondo or the periodic Anderson models as germane to heavy Fermi systems, and also refers occasionally to the $t-J$ class of systems. We use the term ECQL exclusively for systems such as the $t$ - $J$ model, ${ }^{1}$ having much stronger, even extreme correlations built into them. The origin of the difference is the infinite Coulomb repulsion at each site, where double occupancy is prohibited, not just discouraged as in the Hubbard model. The result is that we must deal with a genuinely different problem, both physically and mathematically; here the fundamental operators are no longer canonical fermions but rather Hubbard operators. As we describe below, a systematic theory of the ECQL can be built, in parallel to the Fermi-liquid (FL) theory for the more conventional interacting electron systems. The ECQL is described below as a coupled spin and charge liquid that breaks no symmetry while it accommodates the extreme correlations of the $t-J$ class of models. Its Green's functions and vertex functions can be defined in a specific way that is different and fundamentally more complex than in weakcoupling (FL) models. In this formalism, the instabilities of the ECQL in charge, spin, and superconducting channels can be studied systematically. The extremely correlated quantum liquid described here, is a strong-coupling entity and in contrast to the Fermi liquid, it cannot be related in an adiabatic fashion to a free Fermi gas. ${ }^{2}$ Although the $t$ - $J$ model is obtainable from a noninteracting Fermi gas by turning on the $J$ parameter, one is also obliged to turn on the Hubbard- $U$ term all the way to $U=\infty$. Adiabaticity is lost in the passage to the infinite- $U$ limit; Appendix D illustrates this breakdown of adiabaticity within the context of the atomic limit of the Hubbard model, and outlines the general argument for the invalidity of the Luttinger-Ward theorem in the extreme correlation limit. The ECQL is expected to described the physics for a sufficiently large $U$, and not just $U=\infty$.

The theory of strongly correlated electronic systems, as opposed to the extremely correlated matter studied here, has received considerable attention in recent times. A notable success has been the idea of large dimensionality where the dynamical mean-field theory ${ }^{3,4}$ leads to a controlled set of calculations that are useful as well as predictive. The $t-J$ model, Eq. (1), has also been studied ${ }^{5}$ but perhaps not so widely as the Hubbard model. The origin of the $t$ - $J$ model is the subject of several studies summarized in Ref. 6. We emphasize that it is not merely a descendent of the Hubbard model (upon using a large $U$ expansion) but rather has an independent origin via the downfolding of multiband systems. Thus $t$ and $J$ may be viewed as independent parameters, rather than being fixed through $J=4 t^{2} / U$ as in superexchange theory. The possibility of an expansion of the physical quantities in terms of Mott Hubbard holes, i.e., the departure from half filling, has been anticipated in $1986,{ }^{1}$ but a systematic procedure remained undiscovered. Early studies, ${ }^{1,7}$ have struggled with the technical difficulties of the noncanonical nature of the fermions. The proposal of Anderson $^{8}$ in 1987 , that the high- $T_{c}$ systems are described by this model, has led to a revived and wider interest in this model. Alternate techniques such as the auxiliary field 
method have been employed to deal with the constraints ${ }^{5,9}$ but give divergent views of the outcome of extreme correlations. The present study is motivated by this conundrum, as well as by the possibility of its applicability to certain systems found in nature. The predicted behavior of the ECQL has certain specific signatures that distinguish it from the FL so that it should be relatively straightforward to verify its applicability to a given system.

The plan of the paper is as follows. In Sec. II we present the calculation of the Green's function of the $t-J$ model along with the background definitions of the Hubbard operators and the Schwinger framework. In Sec. III, the exact Schwinger-Dyson equation is given after the sources are turned off. In Sec. IV, we solve for the local Green's function. In Sec. V we outline the computation of the vertices with detailed results given in Appendix G. In Sec. VI the Ward identities are developed and current vertices defined. In Sec. VII, we initiate the program of successive approximations to the Green's function and list several consistent schemes. In Sec. VIII we present a calculation of the $d$-wave superconducting instability of the ECQL using one of the consistent schemes for the Green's functions. In Sec. IX we present a detailed picture of the quasiparticles which are argued to be fractionally charged. The argument for the changed Fermi surface volume is given further in Appendix D. We summarize the results in Sec. X.

Appendix A and Appendices A 1 and A 2, and Appendix $B$ provide the details of the functional derivatives and the functions that arise in that process. Appendix $\mathrm{C}$ gives the details of the Nozières relations that play an important role in giving us rotation invariance of the theory. Appendix E provides the details of different zero source limits that are available. Appendix F contains details of the connection between the quasiparticle susceptibilities and the physical particle susceptibilities as well as some important sum rules that set the scales for the susceptibilities. Appendix $G$ gives all the vertices computed after throwing out higher-order vertices, i.e., $\frac{\delta \Gamma}{\delta V}$. Finally Appendix $\mathrm{H}$ gives the conventions used for various Fourier transforms.

\section{BARE AND QUASIPARTICLE GREEN'S FUNCTIONS OF THE $t$ - $J$ MODEL}

We study the $t$ - $J$ model given by

$$
\begin{aligned}
H= & -\sum_{i, j} t_{i, j} \hat{c}_{\vec{r}_{j}, \sigma}^{\dagger} \hat{c}_{\vec{r}_{i}, \sigma}+\frac{1}{2} \sum_{i, j} J_{i, j}\left\{\vec{S}_{\vec{r}_{i}} \cdot \vec{S}_{\vec{r}_{j}}-\frac{1}{4} n_{\vec{r}_{i}} n_{\vec{r}_{j}}+n_{\vec{r}_{i}}\right\} \\
& -\mu \sum_{i} n_{\vec{r}_{i}},
\end{aligned}
$$

where $\hat{c}_{\vec{r}_{i}, \sigma}^{\dagger}$ creates a electron at the site $i$ which is Gutzwiller projected ${ }^{10}$ to the subspace of single occupancy and the other symbols have their usual meaning. The Gutzwiller projection creates the major technical challenge in this problem. Projected electrons no longer satisfy canonical anticommutation relations and the power of Wick's theorem ${ }^{11}$ is lost, therefore a Dyson equation ${ }^{12}$ with straightforward expansion in terms of a free Green's function is not possible. Lost too are the appealing Feynman diagrams that encapsulate standard FL theory. The projected electrons satisfy instead, a set of graded Lie algebraic commutation relations. The latter are compactly expressed in the notation of Hubbard in terms of the $X$ operators that are summarized in Sec. II A. Section II A contains the details of the definitions of the Green's functions, and of the equations of motion calculation, using the powerful technique invented by Schwinger, Martin and their school. ${ }^{13-15}$ We find that despite the noncanonical nature of the Hubbard operators, we are able to obtain equations that are at the same level of complexity as those of canonical fermions, with some unavoidable embellishments. ${ }^{16}$ We therefore conclude that in the case of projected electrons, the Schwinger-Dyson expansion ${ }^{17}$ is yet possible. $^{18}$ This work studies the resulting equations and their consequences.

\section{A. Basic framework using the Hubbard operators}

Let us define the Hubbard operators acting upon each site as projected Fermi operators

$$
X_{i}^{\sigma_{1} \sigma_{2}}=\left|\sigma_{1}\right\rangle\left\langle\sigma_{2}\left|, \quad X_{i}^{0 \sigma_{1}}=\right| 0\right\rangle\left\langle\sigma_{1}\left|, \quad X_{i}^{\sigma_{1} 0}=\right| \sigma_{1}\right\rangle\langle 0|,
$$

in terms of the three possible states at any site $|0\rangle,|\uparrow\rangle$, and $|\downarrow\rangle$. The doubly occupied site is forbidden and these operators do not connect forbidden states with the allowed ones. Thus an important statement of completeness at any site is the relation

$$
X_{i}^{00}=\mathbb{1}-\sum_{\sigma} X_{i}^{\sigma \sigma}
$$

The first member $X_{i}^{\sigma_{1} \sigma_{2}}$ is bosonic while the other two are fermionic with respect to their commutation relations at different sites. The fundamental anticommutator for the Hubbard operators at different sites is most conveniently expressed as

$$
\left\{X_{i}^{0 \sigma_{1}}, X_{j}^{\sigma_{2} 0}\right\}=\delta_{i j}\left[\delta_{\sigma_{1} \sigma_{2}} X_{i}^{00}+X_{i}^{\sigma_{2} \sigma_{1}}\right]=\delta_{i j}\left[\delta_{\sigma_{1} \sigma_{2}}-\sigma_{1} \sigma_{2} X_{i}^{\bar{\sigma}_{1} \bar{\sigma}_{2}}\right] .
$$

Also note the basic commutator

$$
\left[X_{i}^{0 \sigma_{3}}, X_{j}^{\sigma_{1} \sigma_{2}}\right]=\delta_{i j} \delta_{\sigma_{3} \sigma_{1}} X_{i}^{0 \sigma_{2}}
$$

The Hubbard algebra at a given site is defined by

$$
X_{i}^{a b} X_{i}^{c d}=\delta_{b c} X_{i}^{a d}
$$

In brief we may visualize these Hubbard operators in terms of familiar (canonical) electronic operators $c_{\vec{r}_{i}, \sigma_{a}}^{\dagger}$ creating an electron at site $i$ with spin $\sigma_{a}$, via the nonlinear constructs

$$
\begin{aligned}
& X_{i}^{\sigma_{a}, 0}=\hat{c}_{\vec{r}_{i}, \sigma_{a}}^{\dagger}=\left(1-n_{\vec{r}_{i} \bar{\sigma}_{a}}\right) c_{\vec{r}_{i}, \sigma_{a}}^{\dagger}, \\
& X_{i}^{0, \sigma_{a}}=\hat{c}_{\vec{r}_{i}, \sigma_{a}}=\left(1-n_{\vec{r}_{i} \bar{\sigma}_{a}}\right) c_{\vec{r}_{i}, \sigma_{a},}, \\
& X_{i}^{\sigma_{a}, \sigma_{b}}=\left(1-n_{\vec{r}_{i} \bar{\sigma}_{a}}\right) c_{\vec{r}_{i}, \sigma_{a}}^{\dagger} c_{\vec{r}_{i}, \sigma_{b}},
\end{aligned}
$$

where $\bar{\sigma}_{a}=-\sigma_{a}$ here and throughout this paper. The factors of $\left(1-n_{\vec{r}_{i} \bar{\sigma}_{a}}\right)$ get rid of doubly occupied sites. Although one can work with the $c$ 's and project doubly occupied states out, it is 
optimal to work with the $X$ operators. As Hubbard pointed out, the manifold of lower Hubbard band states defined by the reduced Hilbert space excluding double occupation, is mapped into itself under the action of $X$ 's. by

The Hamiltonian is expressed in terms of the $X$ operators

$$
\begin{aligned}
H= & -\sum_{i, j, \sigma} t_{i j} X_{i}^{\sigma 0} X_{j}^{0 \sigma}-\mu \sum_{i, \sigma} X_{i}^{\sigma \sigma}+\frac{1}{2} \sum_{i, j} J_{i j}\left\{\vec{S}_{i} \cdot \vec{S}_{j}-\frac{1}{4} n_{i} n_{j}\right\} \\
& +\frac{1}{2} \sum_{i, j, \sigma} J_{i j} X_{i}^{\sigma \sigma} .
\end{aligned}
$$

The last (trivial) term is a shift of the chemical potential and is added to make the equations more compact. The third $(J$-dependent) term is rewritten as

$$
\begin{aligned}
V & =\frac{1}{4} \sum_{i j} J_{i j}\left\{P_{i j}-X_{i}^{\sigma_{1} \sigma_{1}} X_{j}^{\sigma_{2} \sigma_{2}}\right\} \\
& =\frac{1}{4} \sum_{i j} J_{i j}\left\{X_{i}^{\sigma_{1} \sigma_{2}} X_{j}^{\sigma_{2} \sigma_{1}}-X_{i}^{\sigma_{1} \sigma_{1}} X_{j}^{\sigma_{2} \sigma_{2}}\right\},
\end{aligned}
$$

where $P$ permutes the spin indices.

\section{B. Calculation of the Green's function}

The Green's function is defined as

$$
\mathcal{G}_{\sigma_{1} \sigma_{2}}[1,2]=-\left\langle T\left[X_{1}^{0 \sigma_{1}}\left(\tau_{1}\right) X_{2}^{\sigma_{2} 0}\left(\tau_{2}\right)\right]\right\rangle,
$$

where $T$ is the time-ordering symbol, and for an arbitrary operator $Q$ we define

$$
\langle Q\rangle=\frac{\operatorname{Tr} e^{-\beta H} Q}{\operatorname{Tr} e^{-\beta H}} .
$$

Thus, $\mathcal{G}$ is a $2 \times 2$ matrix in the spin space. The time dependence is given by

$$
Q(\tau) \equiv e^{\tau H} Q e^{-\tau H} .
$$

In further work we need to add a source term ${ }^{14}$ via the operator $\mathcal{A}$,

$$
\mathcal{A}=\sum_{j, \sigma_{1}, \sigma_{2}} \int_{0}^{\beta} d \tau \mathcal{V}_{j}^{\sigma_{1} \sigma_{2}}(\tau) X_{j}^{\sigma_{1} \sigma_{2}}(\tau)
$$

with the same $\tau$ dependence of the operators as in Eq. (12) and an arbitrary function of time $\mathcal{V}_{j}^{\sigma_{1} \sigma_{2}}(\tau)$ at every site. The Green's functions are now no longer time translation invariant, and are defined as

$$
\mathcal{G}_{\sigma_{1} \sigma_{2}}(1,2)=-\frac{\operatorname{Tr}\left\{e^{-\beta H} T\left[e^{-\mathcal{A}} X_{1}^{0 \sigma_{1}}\left(\tau_{1}\right) X_{2}^{\sigma_{2} 0}\left(\tau_{2}\right)\right]\right\}}{\operatorname{Tr}\left[e^{-\beta H} T\left(e^{-\mathcal{A}}\right)\right]} .
$$

This version of $G$ satisfies the Kubo Martin Schwinger boundary conditions ${ }^{14}$ as may be readily verified

$$
\mathcal{G}_{\sigma_{1} \sigma_{2}}\left[r_{1} 0^{-}, r_{2} 0\right]=-\mathcal{G}_{\sigma_{1} \sigma_{2}}\left[r_{1} \beta, r_{2} 0\right],
$$

so that one may perform a Fourier series with odd integer Matsubara frequencies only. We will use the convention that the Green's function is written as the difference of frequencies after setting the source $\mathcal{A}=0$, whereas for $\mathcal{A} \neq 0$ we will display a function of two separate times.

More generally for any variable we define a modified expectation

$$
\left\langle\left\langle Q\left(\tau_{1}, \tau_{2}, \ldots\right)\right\rangle\right\rangle=\frac{\operatorname{Tr}\left\{e^{-\beta H} T\left[e^{-\mathcal{A}} Q\left(\tau_{1}, \tau_{2}, \ldots\right)\right\}\right.}{\operatorname{Tr}\left[e^{-\beta H} T\left(e^{-\mathcal{A}}\right)\right]}
$$

with a compact notation that includes the time ordering and the exponential factor automatically. Thus

$$
\mathcal{G}_{\sigma_{i} \sigma_{f}}[i, f]=-\left\langle\left\langle X_{i}^{0 \sigma_{i}} X_{f}^{\sigma_{f}{ }^{0}}\right\rangle\right\rangle .
$$

From this the variation in the Green's function can be found from functional differentiation as

$$
\begin{aligned}
\frac{\delta}{\delta \mathcal{V}_{j}^{\sigma_{1} \sigma_{2}}\left(\tau_{1}\right)}\left\langle\left\langle Q\left(\tau_{2}\right)\right\rangle\right\rangle= & \left\langle\left\langle Q\left(\tau_{2}\right)\right\rangle\right\rangle\left\langle\left\langle X_{j}^{\sigma_{1} \sigma_{2}}\left(\tau_{1}\right)\right\rangle\right\rangle \\
& -\left\langle\left\langle X_{j}^{\sigma_{1} \sigma_{2}}\left(\tau_{1}\right) Q\left(\tau_{2}\right)\right\rangle\right\rangle
\end{aligned}
$$

and we note the important commutator

$$
\begin{aligned}
{\left[X_{i}^{0 \sigma_{i}}, H\right]=} & -\sum_{j} t_{i j}\left[\delta_{\sigma_{i} \sigma_{j}}-\sigma_{i} \sigma_{j} X_{i}^{\bar{\sigma}_{i} \bar{\sigma}_{j}}\right] X_{j}^{0 \sigma_{j}}-\mu X_{i}^{0 \sigma_{i}} \\
& +\frac{1}{2} \sum_{j} J_{i j}\left[\delta_{\sigma_{i} \sigma_{j}}-\sigma_{i} \sigma_{j} X_{j}^{\bar{\sigma}_{i} \bar{\sigma}_{j}}\right] X_{i}^{0 \sigma_{j}} .
\end{aligned}
$$

We note the similarity in form between the $t$ and $J$ terms above, one can be transformed into the other by flipping the spatial indices $i, j$ on the $X$ operators; this symmetry persists in the following equations as well. From this point we will use an Einstein-type convention, we sum over all internal repeated indices, while leaving the external indices fixed. By summing over an index, a spatial sum over the lattice and integration over imaginary time $0 \leq \tau_{j} \leq \beta$ is implied. The external indices (both space time and spin) are recognizable since they appear in the left-hand side (LHS) of all the equations.

Let us compute the time derivative of the $G$. For this we need the derivative

$$
\begin{aligned}
\partial_{\tau_{i}} T\left[e^{-\mathcal{A}} X_{i}^{a, b}\left(\tau_{i}\right)\right]= & -T\left\{e^{-\mathcal{A}}\left[X_{i}^{a, b}\left(\tau_{i}\right), H\right]\right\} \\
& +\mathcal{V}_{i}^{\sigma_{1} \sigma_{2}}\left(\tau_{i}\right) T\left\{e^{-\mathcal{A}}\left[X_{i}^{\sigma_{1} \sigma_{2}}\left(\tau_{i}\right), X_{i}^{a, b}\left(\tau_{i}\right)\right]\right\} .
\end{aligned}
$$

This follows from the definition of the time ordering and the form of $\mathcal{A}$. Using this we find

$$
\begin{aligned}
& \partial_{\tau_{i}} \mathcal{G}_{\sigma_{i} \sigma_{f}}[i, f]=-\delta\left(\tau_{i}-\tau_{f}\right) \delta_{i, f}\left\langle\left\langle\left(\delta_{\sigma_{i} \sigma_{f}}-\sigma_{i} \sigma_{f} X_{i}^{\bar{\sigma}_{i} \bar{\sigma}_{f}}\right)\right\rangle\right\rangle \\
& +\left\langle\left\langle\left[X_{i}^{0 \sigma_{i}}\left(\tau_{i}\right), H\right] X_{f}^{\sigma_{f} 0}\left(\tau_{f}\right)\right\rangle\right\rangle-\mathcal{V}_{i}^{\sigma_{i} \sigma_{2}}\left(\tau_{1}\right) \mathcal{G}_{\sigma_{2} \sigma_{f}}[i, f] .
\end{aligned}
$$

We further use the abbreviations,

$$
\begin{array}{cc}
\delta[i, j]=\delta_{i, j} \delta\left(\tau_{i}-\tau_{j}\right), & t[i, j]=t_{i j} \delta\left(\tau_{i}-\tau_{j}\right), \\
J[i, j]=J_{i j} \delta\left(\tau_{i}-\tau_{j}\right), & \mathcal{V}_{r}^{\sigma_{a} \sigma_{b}}=\mathcal{V}_{r}^{\sigma_{a} \sigma_{b}\left[\tau_{r}\right] .}
\end{array}
$$


We next introduce a useful and convenient notion of " $k$ conjugation" of any matrix $M$, denoted by $M^{k}$, such that

$$
\left(M^{k}\right)_{\sigma_{1}, \sigma_{2}}=\sigma_{1} \sigma_{2} M_{\bar{\sigma}_{2}, \bar{\sigma}_{1}} .
$$

This is the time-reversal operation but confined to the spinspace indices and excludes transforming the space-time indices. The $k$ conjugation of a matrix in the spin space produces the transpose of its cofactor matrix, and thus the inverse of any matrix is proportional to its conjugate, as in Eq. (24). More explicitly we see that

$$
\begin{aligned}
& M \cdot M^{k}=\operatorname{det} M 1, \\
& (M)^{-1}=\frac{1}{\operatorname{det} M} M^{k},
\end{aligned}
$$

where 1 is the identity matrix in the $2 \times 2$-dimensional spin space.

We need an object $\Delta[i]$ that plays the role of a dynamical Gutzwiller factor in this theory. It is defined by

$$
\begin{gathered}
\Delta[i]=1-\mathcal{G}^{k}\left[i^{-}, i\right], \\
\Delta_{\sigma_{1} \sigma_{2}}[i]=\delta_{\sigma_{1}, \sigma_{2}}-\sigma_{1} \sigma_{2} \mathcal{G}_{\bar{\sigma}_{2} \bar{\sigma}_{1}}\left[i^{-}, i\right], \\
\Delta_{\sigma_{1} \sigma_{2}}^{-1}[i]=\frac{1}{\operatorname{det} \Delta[i]} \Delta_{\sigma_{1} \sigma_{2}}^{k}[i],
\end{gathered}
$$

where the second and third lines follow from Eq. (23). We also define a matrix functional derivative operator

$$
D_{\sigma_{1}, \sigma_{2}}[r]=\sigma_{1} \sigma_{2} \frac{\delta}{\delta \mathcal{V}_{r}^{\bar{\sigma}_{1} \bar{\sigma}_{2}}} .
$$

In terms of these, we find the equation of motion (EOM)

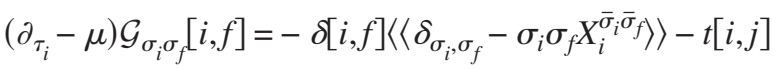

$$
\begin{aligned}
& \times\left\langle\left\langle\left(\delta_{\sigma_{i}, \sigma_{j}}-\sigma_{i} \sigma_{j} X_{i}^{\bar{\sigma}_{i} \bar{\sigma}_{j}}\right) X_{j}^{0 \sigma_{j}}\left(\tau_{i}\right) X_{f}^{\sigma_{f}^{0}}\left(\tau_{f}\right)\right\rangle\right\rangle \\
& +\frac{1}{2} J[i, j]\left\langle\left\langle\left(\delta_{\sigma_{i}, \sigma_{j}}\right.\right.\right. \\
& \left.\left.\left.-\sigma_{i} \sigma_{j} X_{j}^{\bar{\sigma}_{i} \bar{\sigma}_{j}}\right) X_{i}^{0 \sigma_{j}}\left(\tau_{i}\right) X_{f}^{\sigma_{f}^{0}}\left(\tau_{f}\right)\right\rangle\right\rangle \\
& -\mathcal{V}_{i}^{\sigma_{i} \sigma_{j}}\left(\tau_{i}\right) \mathcal{G}_{\sigma_{j} \sigma_{f}}[i, f] \text {. }
\end{aligned}
$$

We employ a useful relation with an arbitrary operator $\mathcal{Q}$,

$$
\left\langle\left\langle\left(\delta_{\sigma_{a} \sigma_{b}}-\sigma_{a} \sigma_{b} X_{a}^{\bar{\sigma}_{a} \bar{\sigma}_{b}}\right) \mathcal{Q}\right\rangle\right\rangle=\left(\Delta_{\sigma_{a} \sigma_{b}}[a]+D_{\sigma_{a} \sigma_{b}}[a]\right)\langle\langle\mathcal{Q}\rangle\rangle,
$$

to rewrite Eq. (26) in component form as

$$
\begin{aligned}
\left(\partial_{\tau_{i}}-\mu\right) \mathcal{G}_{\sigma_{i} \sigma_{f}}[i, f]= & -\delta[i, f] \Delta_{\sigma_{i} \sigma_{f}}[i]-\mathcal{V}_{i}^{\sigma_{i} \sigma_{j}}\left(\tau_{i}\right) \mathcal{G}_{\sigma_{j} \sigma_{f}}[i, f] \\
& +t[i, j]\{(\Delta[i]+D[i]) \cdot \mathcal{G}[j, f]\}_{\sigma_{i} \sigma_{f}} \\
& -\frac{1}{2} J[i, j]\{(\Delta[j]+D[j]) \cdot \mathcal{G}[i, f]\}_{\sigma_{i} \sigma_{f}}
\end{aligned}
$$

This may finally be written compactly in matrix form as

$$
\begin{aligned}
\left(\partial_{\tau_{i}}-\mu\right) \mathcal{G}[i, f]= & -\delta[i, f] \Delta[i]-\mathcal{V}_{i} \cdot \mathcal{G}[i, f]-X[i, j] \cdot \mathcal{G}[j, f] \\
& -Y[i, j] \cdot \mathcal{G}[j, f]
\end{aligned}
$$

where we used the definitions

$$
\begin{aligned}
& X[i, j]=-t[i, j] D[i]+\frac{1}{2} J[i, k] D[k] \delta[i, j], \\
& Y[i, j]=-t[i, j] \Delta[i]+\frac{1}{2} J[i, k] \Delta[k] \delta[i, j] .
\end{aligned}
$$

The space-time indices are displayed but the spin indices are hidden in the above matrix structure.

We next perform a scale (or local-gauge) transformation with a space-time spin-dependent factor described below. This scale transformation is a key step in our work and it is important to appreciate its motivation. If we work with the EOM, Eq. (29), the resulting vertex, i.e., schematically the object $-\frac{\delta}{\delta V} \mathcal{G}^{-1}$, turns out to have pathological "overhangs." By this we mean that the vertex will contain not only $\mathcal{G}$ but also $\mathcal{G}^{-1}$, i.e., the putative "self energy," and hence the resulting Schwinger-Dyson equation will be ill formed. ${ }^{19}$ The origin of the difficulty is that the coefficient of $\delta[i, f]$ in the right-hand side (RHS) of Eq. (29) involves $\Delta[i]$ which is time dependent, and essentially made up of $\mathcal{G}$. This in turn is a manifestation of the noncanonical nature of the projected electrons. The factor $\Delta[i]$ is physically understandable as arising from the spectral weight contained in the lower Hubbard band, which is less than unity. On the other hand, canonical Green's functions contain a spectral weight of unity. These observations are reflected in the coefficient of $\frac{1}{\omega}$ in the limit of high frequencies of the Green's functions changing from unity in the canonical case to $1-n_{\bar{\sigma}}$ for the $t-J$ model.

We resolve this difficulty by a local space-time-dependent scale transformation. With this, we eliminate this inconvenient factor of $\Delta[i]$ by a multiplicative process in the time domain. After the removal, we uncover a new Green's function $\hat{G}$ corresponding to effective canonical electrons or quasiparticles (QP), that lie underneath. One could remove this factor $\Delta[i]$ in many equivalent ways, such as symmetrically or from the right, we choose a left-sided transformation for maximal ease of computation. ${ }^{20}$ Let us write

$$
\mathcal{G}[i, f]=\Delta[i] \cdot \hat{G}[i, f],
$$

so that the EOM, Eq. (29), becomes after some rearrangement

$$
\begin{gathered}
-\delta[i, f] 1=\left\{\left(\partial_{\tau_{i}}-\mu+V_{i}+\Phi_{i}\right) \delta[i, j] 1+\Delta^{-1}[i] \cdot X[i, j] \cdot \Delta[j]\right. \\
\left.+\Delta^{-1}[i] \cdot Y[i, j] \cdot \Delta[j]\right\} \cdot \hat{G}[j, f], \\
V_{i}=\Delta^{-1}[i] \cdot \mathcal{V}_{i} \cdot \Delta[i], \\
\Phi_{i}=\Delta^{-1}[i] \cdot\left(\partial_{\tau_{i}} \Delta[i]\right) .
\end{gathered}
$$

Here $\hat{G}$ is the underlying canonical Green's function (with spectral weight unity), $V$ is the transformed source field, and $\Phi$ arises from the time derivative. Appendix B summarizes 
its properties and demonstrates that it is negligible on turning off the source terms.

\section{Calculation of the inverse Green's function}

We next turn to the task of finding the inverse Green's function. Using the methodology and results detailed in Appendix A [especially Eqs. (34) and (A1)], and with $\vdots Q:$ symbolizing a right (i.e., normal) ordering of the functional derivative contained in the matrix operator $Q$, we may rewrite Eq. (32) as

$$
\begin{aligned}
-\delta[i, f] \mathbb{1}= & \left(\partial_{\tau_{i}}-\mu+V_{i}+\Phi_{i}\right) \cdot \hat{G}[i, f]-t[i, j] \Delta[j] \cdot \hat{G}[j, f] \\
& +\frac{1}{2} J[i, k] \mu[i, k] \cdot \Delta[i] \cdot \hat{G}[i, f]-t[i, j](\nu[i, j] \\
& +\vdots \mathcal{D}[i] \cdot \mu[i, j] \vdots) \cdot \hat{G}[j, f]+\frac{1}{2} J[i, k] \\
& \times(\mu[i, k] \cdot \nu[k, i] \\
& +\vdots \mu[i, k] \cdot \mathcal{D}[k] \cdot \mu[k, i] \vdots) \cdot \hat{G}[i, f] .
\end{aligned}
$$

Here we denote

$$
\begin{gathered}
\mu[i, j]=\Delta^{-1}[i] \cdot \Delta[j], \\
\nu[k, j]=\overline{\vdots \mathcal{D}[j] \cdot \Delta^{-1}[j] \vdots \cdot \Delta[k]}, \\
\mathcal{D}_{\sigma_{1}, \sigma_{2}}[i]=\sigma_{1} \sigma_{2} \frac{\delta}{\delta V_{i}^{\bar{\sigma}_{1} \bar{\sigma}_{2}}} .
\end{gathered}
$$

The matrix products are indicated by the center dots and the terms under the overline symbol indicate the extent of terms over which the derivative acts. Using the results detailed in Appendix A Eq. (A3) and Sec. IV Eq. (62), we express various objects in terms of $\hat{G}$ (rather than $\mathcal{G}$ )

$$
\Delta[j]=\frac{1}{\gamma[j]}\left(1-\hat{G}^{k}\left[j^{-}, j\right]\right),
$$

where

$$
\gamma[j]=1-\operatorname{det}\left[\hat{G}\left(j^{-}, j\right)\right] .
$$

With these identifications, we have converted the problem to one only involving $\hat{G}$ and $V$ at this point, and jettisoned all reference to the original Green's function $\mathcal{G}$ and the original source $\mathcal{V}$.

Our next aim is to find an equation for the inverse ${ }^{21}$ of $\hat{G}$ defined through

$$
\begin{gathered}
\hat{G}[i, j] \cdot \hat{G}^{-1}[j, k]=\hat{G}^{-1}[i, j] \cdot \hat{G}[j, k]=1 \delta[i, k], \\
\hat{G}_{\sigma_{1}, \sigma_{2}}[i, j] \hat{G}_{\sigma_{2}, \sigma_{3}}^{-1}[j, k]=\delta_{\sigma_{1}, \sigma_{3}} \delta[i, k] .
\end{gathered}
$$

It is useful to define a "susceptibility"-type three point object $\chi$,

$$
\chi_{\sigma_{c} \sigma_{d}}^{\sigma_{a} \sigma_{b}}[p, q ; r] \equiv \frac{\delta \hat{G}_{\sigma_{a} \sigma_{b}}[p, q]}{\delta V_{r}^{\sigma_{c} \sigma_{d}}},
$$

and a vertex function $\Gamma$,

$$
\Gamma_{\sigma_{c} \sigma_{d}}^{\sigma_{a} \sigma_{b}}[p, q ; r]=-\frac{\delta \hat{G}_{\sigma_{a} \sigma_{b}}^{-1}[p, q]}{\delta V_{r}^{\sigma_{c} \sigma_{d}}},
$$

so that

$$
\chi_{\sigma_{c} \sigma_{d}}^{\sigma_{a} \sigma_{b}}[p, q ; r]=\hat{G}_{\sigma_{a} \sigma_{1}}[p, x] \Gamma_{\sigma_{c} \sigma_{d}}^{\sigma_{1} \sigma_{2}}[x, y ; r] \hat{G}_{\sigma_{2} \sigma_{b}}[y, q]
$$

The relations of these susceptibilities with the physical ones are detailed in Appendix F. Let us right multiply Eq. (33) by $\hat{G}^{-1}[f, m]$ (and sum over $f$ ) so that

$$
\begin{aligned}
& -\hat{G}^{-1}[i, m]=\left(\partial_{\tau_{i}}-\mu+V_{i}+\Phi_{i}\right) \delta[i, m] 1 \\
& -t[i, m](\nu[i, m]+\Delta[m])+\frac{1}{2} J[i, k] \\
& \times(\mu[i, k] \cdot \nu[k, i]+\mu[i, k] \cdot \Delta[i]) \delta[i, m] \\
& -t[i, j] \overline{\vdots \mathcal{D}[i] \cdot \mu[i, j] \vdots \cdot \hat{G}[j, f]} \cdot \hat{G}^{-1}[f, m] \\
& +\frac{1}{2} J[i, k] \overline{\vdots \mu[i, k] \cdot \mathcal{D}[k] \cdot \mu[k, i] \vdots \cdot \hat{G}[i, f]} \\
& \text { - } \hat{G}^{-1}[f, m] \text {. }
\end{aligned}
$$

More explicitly, the derivative excludes operating upon the factor $\hat{G}^{-1}[f, m]$ since the overline excludes that term. The detailed calculation is presented in Appendix A. We use Eqs. (A10), (A14), and (A15) to rewrite this expression. We split the Green's function into the following form with $\hat{G}_{0}$ as the bare quasiparticle Green's function and $\mathcal{S}$ as the quasiparticle self-energy,

$$
\hat{G}^{-1}[i, j]=\hat{G}_{0}^{-1}[i, j]-\mathcal{S}[i, j],
$$

where

$$
\begin{aligned}
-\hat{G}_{0}^{-1}[i, j] \equiv & {\left[\partial_{\tau_{i}}-\mu+V_{i}+\frac{1}{2}\left(\sum_{k} J_{i, k}\right)\left(1-\frac{n}{2}\right)\right] \delta[i, j] } \\
& -t[i, j]\left(1-\frac{n}{2}\right) .
\end{aligned}
$$

The self-energy is given by

$$
\begin{aligned}
\mathcal{S}[i, j]= & \Phi_{i} \delta(i, j)-t[i, j]\left\{\left[\Delta[j]-\left(1-\frac{n}{2}\right)\right]+\nu[i, j]\right\} \\
& -t[i, k] \Theta[i, k, j]+\delta[i, j] \frac{1}{2} J[i, k][\mu[i, k] \cdot \Delta[i] \\
& \left.-\left(1-\frac{n}{2}\right)\right]+\delta[i, j] \frac{1}{2} J[i, k] \mu[i, k] \cdot \nu[k, i] \\
& +\frac{1}{2} J[i, k] \mu[i, k] \cdot \Theta[k, i, j] .
\end{aligned}
$$

Regarding nomenclature, we call the expression $\hat{G}_{0}$ as the 
bare, rather than the unperturbed, Green's function, and the rest as self-energy, since there is no perturbation theory involved here. The result, Eqs. (42) and (43), is the exact Schwinger-Dyson equation including the source field, and is central to the calculation of the vertices. Calculating the vertices proceeds by taking functional derivatives of Eq. (43), and leads to a standard hierarchy of equations for higherorder vertices. ${ }^{13,15}$ We should also clarify that in contrast to the nomenclature of Fermi liquids, the quasiparticles as defined here are not infinitely sharp in energy, they decay through relaxation processes that we describe via the imaginary part of their (analytically continued) self-energy. Thus the quasiparticles of the ECQL have a coherent part as well as an incoherent background part that emerges in complete analogy to the electron Green's function in the FL.

\section{SCHWINGER DYSON EQUATION AND THE LIMIT OF VANISHING SOURCES}

Let us gather various objects here when the sources are turned off, i.e., $V \rightarrow 0$. We recover all the symmetries in this limit, translation invariance allows us to perform Fourier transforms in space and time, and rotation invariance leads to important simplifications of the vertices and susceptibilities. Due to the rotation invariance of the extremely correlated quantum liquid, the three triplet states of the particle-hole pair are degenerate. This leads to important identities that parallel the relations in the Fermi liquid. We denote these as Nozières relations and discuss them further in Appendix C, where the abbreviated superscripts and subscripts of the vertices are defined. Therefore as $V \rightarrow 0$,

$$
\begin{gathered}
\mathcal{G}_{\sigma_{1}, \sigma_{2}}[i, j] \rightarrow \delta_{\sigma_{1}, \sigma_{2}} \mathcal{G}[i-j], \quad \hat{G}_{\sigma_{1}, \sigma_{2}}[i, j] \rightarrow \delta_{\sigma_{1}, \sigma_{2}} \hat{G}[i-j], \\
\Delta_{\sigma_{1}, \sigma_{2}}[k] \rightarrow \delta_{\sigma_{1}, \sigma_{2}}\left(1-\frac{n}{2}\right), \quad \mu_{\sigma_{1}, \sigma_{2}}[k, l] \rightarrow \delta_{\sigma_{1}, \sigma_{2}}
\end{gathered}
$$

and for any object $Q=\chi, Y$, or $\Gamma$ we have a spin decomposition

$$
\begin{gathered}
Q_{\sigma_{3}, \sigma_{4}}^{\sigma_{1}, \sigma_{2}} \rightarrow \delta_{\sigma_{1}, \sigma_{2}} \delta_{\sigma_{3}, \sigma_{4}}\left\{\delta_{\sigma_{1}, \sigma_{3}} Q^{(1)}+\delta_{\sigma_{1}, \bar{\sigma}_{3}} Q^{(2)}\right\} \\
+\delta_{\sigma_{1}, \sigma_{3}} \delta_{\sigma_{2}, \sigma_{4}} \delta_{\sigma_{1}, \bar{\sigma}_{2}} Q^{(3)} \\
Q_{t}=Q^{(1)}-Q^{(2)}=Q^{(3)} \\
Q_{s}=Q^{(1)}+Q^{(2)}
\end{gathered}
$$

The object $Q_{s}$ refers to the particle-hole singlet channel, corresponding to a charge-density variable and $Q_{t}$ to the particle-hole triplet channel, i.e., a spin-density variable.

Let us note that in the limit of vanishing sources, the physical projected electron Green's function $\mathcal{G}$ and the quasiparticle Green's function $\hat{G}$ are simply related as

$$
\mathcal{G}[i-j]=\left(1-\frac{n}{2}\right) \hat{G}[i-j]
$$

and

$$
\mathcal{G}\left[\vec{k}, i \omega_{n}\right]=\left(1-\frac{n}{2}\right) \hat{G}\left[\vec{k}, i \omega_{n}\right]
$$

The object $\nu$ is now a function of the difference $a-b$, and due to the multiplying factors $t[a, b], J[a, b]$ in the expression for $\hat{G}$, we usually need this at equal times $\tau_{a}=\tau_{b}$. We Fourier transform using the convention in Appendix $\mathrm{H}$ as

$$
\begin{aligned}
\nu_{\sigma_{1}, \sigma_{2}}[a, b]= & \delta_{\sigma_{1}, \sigma_{2}} \nu[a, b]=\sum_{p} \nu[q] \exp -i(a-b) q, \\
\nu[a, b]= & \frac{1-\frac{n}{2}}{1-n}\left\{\left(\frac{n}{2}-1\right) \chi^{(1)}[a, a ; b]+\frac{n}{2} \chi^{(2)}[a, a ; b]\right. \\
& \left.-\chi^{(3)}[a, a ; b]\right\} \\
= & \frac{1}{n}\left\{\left\langle\vec{S}_{a} \cdot \vec{S}_{b}\right\rangle+\frac{1}{4}\left(\left\langle n_{a} n_{b}\right\rangle-n^{2}\right)\right\}, \quad \text { with } \tau_{a}=\tau_{b}, \\
& \left.1-\frac{1}{2}\right\} \\
& \nu[q]=-\frac{1}{2}\left(1-\frac{n}{2}\right) \chi_{s}[q]-\frac{3}{2} \frac{1-\frac{n}{2}}{1-n} \chi_{t}[q] .
\end{aligned}
$$

Further on turning off the sources, we find from Appendix A 2

$$
\begin{aligned}
\Theta_{\sigma_{1}, \sigma_{2}}[r, s, m] & =\sigma_{1} \sigma_{a} \mu_{\sigma_{a}, \sigma_{b}}[r, s] \hat{G}_{\sigma_{b}, \sigma_{c}}[s, k] \Gamma_{\bar{\sigma}_{1}, \bar{\sigma}_{a}}^{\sigma_{c}, \sigma_{2}}[k, m ; r] \\
& \rightarrow \delta_{\sigma_{1}, \sigma_{2}} G[s, k]\left\{\Gamma^{(2)}[k, m ; r]-\Gamma^{(3)}[k, m ; r]\right\} \\
& =\delta_{\sigma_{1}, \sigma_{2}} G[s, k] \Gamma^{(p)}[k, m, r]
\end{aligned}
$$

As explained in Appendix C, the superscript $(p)$ stands for Cooper-pairing channel and is a specific linear superposition of the singlet and triplet channels $\Gamma^{(p)}=\frac{1}{2}\left(\Gamma_{s}-3 \Gamma_{t}\right)$. Hence we may write the EOM as

$$
\begin{aligned}
-\hat{G}^{-1}[i, j]= & \left(\partial_{\tau_{i}}-\mu+\Phi_{i}\right) \delta[i, j]-t[i, j]\left[\left(1-\frac{n}{2}\right)+\nu[i, j]\right] \\
& -t[i, l] G[l, k] \Gamma^{(p)}[k, j ; i] \\
& +\frac{1}{2} J[i, k]\left[\nu[k, i]+\left(1-\frac{n}{2}\right)\right] \delta[i, j] \\
& \left.+\frac{1}{2} J[i, k] G[i, l] \Gamma^{(p)}[l, j ; k]\right) .
\end{aligned}
$$

Using the convention for Fourier transforms in Appendix $\mathrm{H}$, and with the Fourier space version of $\hat{G}_{0}$, Eq. (42),

$$
\hat{G}_{0}^{-1}[k]=i \omega_{k}+\mu-\left(1-\frac{n}{2}\right)\left(\varepsilon_{k}+\frac{1}{2} J_{0}\right)
$$

we write the exact Schwinger-Dyson equation for the Green's function

$$
\hat{G}^{-1}[k]=\hat{G}_{0}^{-1}[k]-\mathcal{S}[k],
$$




$$
\begin{aligned}
\mathcal{S}[k]= & \sum_{q}\left(\varepsilon_{q+k}+\frac{1}{2} J_{q}\right) \nu[q]+\frac{1}{2} \sum_{q}\left(\varepsilon_{q}+\frac{1}{2} J_{k-q}\right) \hat{G}[q] \\
& \times\left\{\Gamma_{s}[q, k]-3 \Gamma_{t}[q, k]\right\} .
\end{aligned}
$$

The self-energy explicitly contains the electron dispersion $\varepsilon_{q}$ or $t_{i j}$ as a multiplicative factor here. This is quite unlike the standard case of the electron liquid, where the entire dependence on the electronic dispersion is hidden in the form of the bare propagator $G_{0}$, and the self-energy is a functional of only the (full) Green's function. ${ }^{15,22-24}$

In order to evaluate the first term of $\mathcal{S}$ in Eq. (51), we need a few definitions at this point. Let us denote

$$
\begin{aligned}
& \chi_{s}[Q]=\sum_{q} \chi_{s}[q, q+Q], \quad \chi_{\mathrm{loc}}=\sum_{Q} \chi_{s}[Q]=-\frac{n}{2} \frac{(1-n)}{\left(1-\frac{n}{2}\right)^{2}}, \\
& \varepsilon_{p}^{s}=\frac{1}{\chi_{\mathrm{loc}} Q} \sum_{p+Q} \chi_{s}[Q], \quad J_{p}^{s}=\frac{1}{\chi_{\mathrm{loc}} Q} \sum_{p+Q} \chi_{s}[Q] .
\end{aligned}
$$

Similar definitions hold in the triplet channel. In Appendix F we show that the local object $\chi_{\mathrm{loc}}$ is identical for both triplet and singlet channels. From Eqs. (47), (51), and (52), and further using the definition of the renormalized energy-type variables

$$
\begin{aligned}
& \hat{E}_{k}=\sum_{q} \varepsilon_{q+k} \nu[q]=\frac{3}{4} \frac{n}{\left(1-\frac{n}{2}\right)} \varepsilon_{k}^{t}+\frac{1}{4} \frac{n(1-n)}{\left(1-\frac{n}{2}\right)} \varepsilon_{k}^{s}, \\
& \mathcal{J}_{k}=\frac{1}{2} \sum_{q} J_{q+k} \nu[q]=\frac{3}{4} \frac{n}{\left(1-\frac{n}{2}\right)} J_{k}^{t}+\frac{1}{4} \frac{n(1-n)}{\left(1-\frac{n}{2}\right)} J_{k}^{s}
\end{aligned}
$$

we find

$$
\begin{aligned}
\mathcal{S}[k]= & \left(\hat{E}_{k}+\frac{1}{2} \mathcal{J}_{0}\right)+\frac{1}{2} \sum_{q}\left(\varepsilon_{q}+\frac{1}{2} J_{k-q}\right) \hat{G}[q]\left\{\Gamma_{s}[q, k]\right. \\
& \left.-3 \Gamma_{t}[q, k]\right\} .
\end{aligned}
$$

By construction, the variables $J_{p}^{s}, J_{p}^{t}, \varepsilon_{p}^{s}$, and $\varepsilon_{p}^{t}$ are weighted averages of the bare objects $J_{p}, \varepsilon_{p}$ with momentumdependent but static weight factors, and hence it is reasonable to view them as correlation adjusted versions of the bare dispersions. The precise relation between the bare dispersions and $\hat{E}_{k}, \mathcal{J}_{k}$ is particularly simple when the hopping is only nearest neighbor; it involves the spin-spin and densitydensity correlation functions at nearest-neighbor distances, and is given explicitly below in Eq. (58). Specializing to only nearest-neighbor hoppings, we see from symmetry that the form of the band dispersion remains a simple tightbinding one. Therefore we write a convenient notation of various objects for the simple cubic lattices

$$
x_{t, s} \equiv \frac{1}{\chi_{\mathrm{loc}}} \sum_{q} \cos q_{x} \chi_{t, s}[q] .
$$

The parameters $x_{s}$ and $x_{t}$ can be found in terms of equal-time correlations by carrying out the frequency and spatial sums, and using Eqs. (F5) and (F8), we find

$$
x_{s}=\frac{\left\langle n_{\vec{r}_{i}} n_{\vec{r}_{i}+\vec{\eta}}\right\rangle-n^{2}}{n-n^{2}}, \quad \text { and } \quad x_{t}=\frac{4}{3 n}\left\langle\vec{S}_{\vec{r}_{i}} \cdot \vec{S}_{\vec{r}_{i}+\vec{\eta}}\right\rangle
$$

where $\vec{\eta}$ is a nearest-neighbor vector. In terms of these, we may write

$$
\varepsilon_{k}^{t}=x_{t} \varepsilon_{k}, \quad \varepsilon_{k}^{s}=\varepsilon_{s} J_{k}, \quad J_{k}^{t}=x_{t} J_{k}, \quad J_{k}^{s}=x_{s} J_{k} .
$$

Thus we can express

$$
\begin{aligned}
& \hat{E}_{k}=\frac{\varepsilon_{k}}{1-\frac{n}{2}}\left\{\left\langle\vec{S}_{\overrightarrow{0}} \cdot \vec{S}_{\vec{\eta}}\right\rangle+\frac{1}{4}\left(\left\langle n_{0} n_{\vec{\eta}}\right\rangle-n^{2}\right)\right\}, \\
& \mathcal{J}_{k}=\frac{J_{k}}{1-\frac{n}{2}}\left\{\left\langle\vec{S}_{\overrightarrow{0}} \cdot \vec{S}_{\vec{\eta}}\right\rangle+\frac{1}{4}\left(\left\langle n_{0} n_{\vec{\eta}}\right\rangle-n^{2}\right)\right\},
\end{aligned}
$$

where $\vec{\eta}$ is a nearest-neighbor vector and the correlations are at equal times. Thus the nearest-neighbor charge and spin correlations directly influence the effective bandwidth. The spin correlation has its largest magnitude near half filling, and can be positive or negative depending upon whether ferromagnetic (FM), i.e., Nagaoka-Thouless-type correlations, or the more usual antiferromagnetic correlations prevail. Near half filling the density term in Eq. (58) is suppressed while the spin-correlation term survives and gives the dominant contribution. This correction term to the energy dispersion $\hat{E}$ is of the same form as $\varepsilon$ but has the opposite sign and hence leads to an important band narrowing via the correlation functions indicated in Eq. (58) in this theory.

Later we will find equations for the susceptibilities so that these correlation functions can be found in terms of the vertices and the Green's functions self-consistently. Successive approximations for the vertices will be formulated with each approximation providing a complete calculational scheme. We will find that the susceptibilities $\chi_{s, t}[Q]$ vanish generically as $1-n$ near half filling, in a similar fashion as $\chi_{\text {loc }}$, and hence these renormalized energies tend to a finite nonzero limit at half filling.

Equation (54) is the exact Schwinger-Dyson equation for the $t-J$ model. This breakup of the inverse $\hat{G}$ into $\hat{G}_{0}$ and a self-energy-type object $\mathcal{S}$ is to some extent arbitrary since there is no a priori notion of an unperturbed Green's function. Our breakup has the natural advantage that the resulting bare vector vertices are frequency independent and in agreement with independent arguments [see next section where the bare current vertex is obtained in Eq. (103)]. Further they satisfy the Ward identities as noted below, Eq. (97). The $\mathcal{S}$ object, after the standard analytic continuation to the retarded self-energy, provides a correction to the quasiparticle energies at the poles of $\hat{G}$ through its real part and more impor- 
tantly to the decay of the quasiparticles through its imaginary part. The self-energy in our formulation is developed in terms of the Green's function $\hat{G}$ and is explicitly a functional of $\hat{G}$. However, and in contrast to the usual weakly interacting Fermi system formulation, ${ }^{22,23,25,26}$ it is not a universal functional, i.e., it does depend upon the bare dispersion $t_{i j}, J_{i j}$ regardless of how we define the self-energy.

Finally it is important to note that this Schwinger-Dyson equation is well formed in the sense that the vertices $\Gamma$ are guaranteed to be well behaved (i.e., finite) for $\omega \rightarrow \infty$, since we have avoided the linear $\omega$ dependence of $\Gamma$ that results, if one does not extract the time-dependent factor $\Delta[i]$ as in Eq. (31). The equations for the vertices are derived and discussed below.

\section{SOLUTION OF THE LOCAL GREEN'S FUNCTION}

We next consider the various local Green's functions and their interrelations. Start from

$$
\mathcal{G}\left[j^{-}, j\right]=\Delta[j] \cdot \hat{G}\left[j^{-}, j\right]
$$

with

$$
\Delta[j]=1-\mathcal{G}^{k}\left[j^{-}, j\right],
$$

so that

$$
\Delta[j]=1-\hat{G}^{k}\left[j^{-}, j\right] \cdot \Delta^{k}[j]
$$

This equation is easy to solve when we iterate once,

$$
\Delta[j]=1-\hat{G}^{k}\left[j^{-}, j\right]+\hat{G}^{k}\left[j^{-}, j\right] \cdot \Delta[j] \cdot \hat{G}\left[j^{-}, j\right],
$$

we see that the inhomogeneous term $1-\hat{G}^{k}$ commutes with $\Delta$ iteratively, so that we may as well rewrite the second term in the RHS as $\Delta[j] \cdot \hat{G}^{k}\left[j^{-}, j\right] \cdot \hat{G}\left[j^{-}, j\right]=\operatorname{det}[G] \Delta[j]$, whereby the solution is noted in Eq. (37),

$$
\Delta[j]=\frac{1}{\gamma[j]}\left(1-G^{k}\left[j^{-}, j\right]\right),
$$

where

$$
\gamma[j]=1-\operatorname{det}\left[G\left(j^{-}, j\right)\right] .
$$

We also require the inverse

$$
\Delta^{-1}[j]=\frac{\gamma[j]}{\gamma 1[j]}\left(1-G\left[j^{-}, j\right]\right),
$$

where

$$
\gamma 1[j]=\operatorname{det}\left[\mathbb{W} 1-G\left(j^{-}, j\right)\right]
$$

These local objects are the dynamical analogs of the Gutzwiller projection factors, ${ }^{10}$ and we note their physical meaning in terms of the bare (quasiparticle) charges $n[i]$ $\left(n_{\mathrm{QP}}[i]\right)$ and spin densities $\vec{S}[i]\left(\vec{S}_{\mathrm{QP}}[i]\right)$ defined in Eqs. (144) and (151)

$$
\begin{aligned}
\gamma[j] & =\frac{1-n[i]}{\left(1-\frac{1}{2} n[i]\right)^{2}-\vec{S}[i] \cdot \vec{S}[i]} \\
& =1-\frac{1}{4}\left(n_{\mathrm{QP}}[i]\right)^{2}+\vec{S}_{\mathrm{QP}}[i] \cdot \vec{S}_{\mathrm{QP}}[i], \\
\gamma 1[j] & =\frac{(1-n[i])^{2}}{\left(1-\frac{1}{2} n[i]\right)^{2}-\vec{S}[i] \cdot \vec{S}[i]} \\
& =\left(1-\frac{1}{2} n_{\mathrm{QP}}[i]\right)^{2}-\vec{S}_{\mathrm{QP}}[i] \cdot \vec{S}_{\mathrm{QP}}[i] .
\end{aligned}
$$

When we turn off the sources, the ECQL state has the following local Green's functions expressed in terms of the number density at each site per spin $n=N /\left(2 N_{\text {sites }}\right)$,

$$
\mathcal{G}\left[j^{-}, j\right]=\frac{n}{2}, \quad \hat{G}\left[j^{-}, j\right]=\frac{\frac{n}{2}}{1-\frac{n}{2}}, \quad \Delta[j]=\left(1-\frac{n}{2}\right),
$$

$$
\gamma[j]=\frac{1-n}{\left(1-\frac{n}{2}\right)^{2}} \quad \gamma 1[j]=\frac{(1-n)^{2}}{\left(1-\frac{n}{2}\right)^{2}} .
$$

The vanishing near half filling of $\gamma$ and $\gamma 1$, as $(1-n)$ and $(1-n)^{2}$ has important consequences, and leads to the holedensity expansion reported below in Sec. VII E.

\section{CALCULATION OF THE VERTICES}

The vertices can be obtained from the general equations for the Green's function, Eqs. (42) and (43), as

$$
\begin{gathered}
\Gamma^{(1)}[i, j ; k]=\delta[i, j] \delta[i, k]+\left(\frac{\delta}{\delta V_{k}^{\uparrow \uparrow}} \mathcal{S}_{\uparrow \uparrow}[i, j]\right)_{V \rightarrow 0}, \\
\Gamma^{(2)}[i, j ; k]=\left(\frac{\delta}{\delta V_{k}^{\downarrow \downarrow}} \mathcal{S}_{\uparrow \uparrow}[i, j]\right)_{V \rightarrow 0}, \\
\Gamma^{(3)}[i, j ; k]=\Gamma^{(1)}[i, j ; k]-\Gamma^{(2)}[i, j ; k] .
\end{gathered}
$$

Thus the bare vertices are simple, writing them in Fourier space we find

$$
\Gamma_{s}\left[p_{1}, p_{2}\right] \rightarrow 1, \quad \Gamma_{t}\left[p_{1}, p_{2}\right] \rightarrow 1 .
$$

The vertex corrections arise from the self-energy part of Eq. (64) and contain several terms. We provide the results of the long calculation in Appendix G. There all terms are retained, with the proviso that the higher-order vertex, obtained by differentiating the three-point vertex are set to zero, i.e., $\frac{\delta \Gamma}{\delta V} \rightarrow 0$.

While this complete solution is available in Appendix G, it is crucial to understand the relative order of various terms, in order to make sensible approximations. We next provide a 
set of calculations that give us such an understanding, we evaluate the derivatives of the basic elements $\Delta[i], \mu[i, j]$, and $\nu[i, j]$ occurring in the self-energy, and show that these have an explicit dependence upon the hole density $\delta=1-n$, measured from half filling. The terms can in fact be grouped in a formal expansion in the inverse hole density $\lambda=\frac{1}{1-n}$. In addition there is an implicit dependence on $n$ in all terms, and certain terms vanish with $\delta$. Thus the final result for the vertex obtained by taking a product of the apparently divergent terms and the coefficients thereof either vanish at half filling $n=1$ or remain finite. This gives us an organizing principle for grouping the contributions, the leading terms near half filling consist of terms that remain finite at $n=1$, and one can throw out the rest, thereby giving us a hole-density expansion that has been conjectured earlier. ${ }^{1}$

Therefore taking the derivatives of the Schwinger-Dyson equation with sources, Eq. (43), we find that the expansion of the vertices can be arranged as a formal power series in the inverse hole density $\lambda$, in the form $\Gamma=1+\Gamma_{0}+\lambda \Gamma_{1}+\lambda^{2} \Gamma_{2}$. It is implied that the coefficients $\Gamma_{j}$ contain terms that are either vanishing at half filling or are finite, and rules for recognizing this implicit dependence are provided later. In Appendix $G$ we list the vertices $\Gamma_{0}, \Gamma_{1}$, and $\Gamma_{2}$.

The existence of this expansion is fortunate since the most interesting physical regime for a doped Mott insulator is in the limit $n \rightarrow 1$. The factors of $\lambda$ arise from the dynamical projection factors $\Delta[i], \mu[i, j]$, and $\nu[i, j]$ in the local Green's functions and the self-energy. We next list the leading behavior of the functional derivatives of the various matrix functions $\mu[i, j], \nu[i, j]$, and $\Delta[i]$ w.r.t. the source terms, so that results for the vertices can be assembled together. For this section, we will use spin diagonal sources $V_{m}$ so that the matrices can all be taken as diagonal in spin space. We will need to use the Noziéres relations $\chi^{(1)} \pm \chi^{(2)}=\chi_{s, t}$ in order to obtain the complete the set of derivatives in the singlet and triplet channels. After providing the results, we will discuss their relative magnitudes, and also the implicit density dependence of the coefficients.

Let us begin with the less singular terms $\Delta$ and $\mu$, where the functional derivative is explicitly $O\left(\frac{1}{\delta}\right)$, and then progress to $\nu$ which is $O\left(\frac{1}{\delta^{2}}\right)$. At some places, we will use the symbol $\sim$ to indicate that the density $n$ has been set at unity in all terms except the singular $\frac{1}{(1-n)^{a}}$-type terms.

\section{A. $\Delta[i]$ and its derivatives}

Let us recall for spin diagonal sources (Appendix E)

$$
\begin{aligned}
\Delta_{\uparrow \uparrow}[i] & =\frac{1}{\gamma[i]}\left(1-G_{\downarrow \downarrow}\left[i^{-}, i\right]\right),\left.\quad \Delta_{\uparrow \uparrow}[i]\right|_{V \rightarrow 0}=\left(1-\frac{n}{2}\right), \\
\gamma[i] & =1-\hat{G}_{\uparrow \uparrow}\left[i^{-}, i\right] \hat{G}_{\downarrow \downarrow}\left[i^{-}, i\right],\left.\quad \frac{\delta \gamma[i]}{\delta V_{m}^{\sigma \sigma}}\right|_{V \rightarrow 0} \\
& =-\frac{n}{2-n} \chi_{s}\left[i^{-}, i ; m\right] .
\end{aligned}
$$

Therefore

$$
\begin{aligned}
\left.\frac{\delta \Delta_{\uparrow \uparrow}(i)}{\delta V_{m}^{\uparrow \uparrow}}\right|_{V \rightarrow 0} & =\frac{\left(1-\frac{n}{2}\right)^{2}}{(1-n)}\left[\frac{n}{2} \chi_{s}\left[i^{-}, i ; m\right]-\chi^{(2)}\left[i^{-}, i ; m\right]\right] \\
& \sim \frac{1}{8 \delta} \chi_{t}\left[i^{-}, i ; m\right], \\
\left.\frac{\delta \Delta_{\uparrow \uparrow}[i]}{\delta V_{m}^{\downarrow \downarrow}}\right|_{W \rightarrow 0} & =\frac{\left(1-\frac{n}{2}\right)^{2}\left[\frac{n}{2} \chi_{s}\left[i^{-}, i ; m\right]-\chi^{(1)}\left[i^{-}, i ; m\right]\right]}{} \\
& \sim-\frac{1}{8 \delta} \chi_{t}\left[i^{-}, i ; m\right] .
\end{aligned}
$$

\section{B. $\mu[i, k]$ and its derivatives}

Lets us recall $\mu[i, k]=\Delta^{-1}[i] \cdot \Delta[k]$, and it may be expressed as

$$
\mu_{\uparrow \uparrow}[i, k]=g_{2}[k, i]\left(1-\hat{G}_{\uparrow \uparrow}\left[i^{-}, i\right]\right)\left(1-\hat{G}_{\downarrow \downarrow \downarrow}\left[k^{-}, k\right]\right),
$$

where $g_{2}[i, j]$ and a related object $g_{1}[i, j]$ needed in the next section are given by

$$
\begin{gathered}
g_{1}[i, j]=\frac{\gamma[j]}{\gamma 1[j] \gamma[i]^{2}}, \quad g_{2}[i, j]=\frac{\gamma[j]}{\gamma 1[j] \gamma[i]}, \\
\left.g_{1}[i, j]\right|_{V \rightarrow 0}=\frac{\left(1-\frac{n}{2}\right)^{4}}{(1-n)^{3}},\left.\quad g_{2}[i, j]\right|_{V \rightarrow 0}=\frac{\left(1-\frac{n}{2}\right)^{2}}{(1-n)^{2}},
\end{gathered}
$$

where we used the definitions [recall Eqs. (37) and (62)].

We first note the derivatives of $g$,

$$
\begin{aligned}
\left.\frac{\delta g_{1}[i, j]}{\delta V_{m}^{\sigma \sigma}}\right|_{V \rightarrow 0}= & \frac{\left(1-\frac{n}{2}\right)^{5}}{(1-n)^{4}}\left[n \chi_{s}\left[i^{-}, i ; m\right]\right. \\
& \left.+\left(1-\frac{n}{2}\right) \chi_{s}\left[j^{-}, j ; m\right]\right] \\
& \sim \frac{1}{64 \delta^{4}}\left[2 \chi_{s}\left[i^{-}, i ; m\right]+\chi_{s}\left[j^{-}, j ; m\right]\right],
\end{aligned}
$$

$$
\begin{aligned}
\left.\frac{\delta g_{2}[i, j]}{\delta V_{m}^{\sigma \sigma}}\right|_{V \rightarrow 0}= & \frac{\left(1-\frac{n}{2}\right)^{3}}{(1-n)^{3}}\left[\frac{n}{2} \chi_{s}\left[i^{-}, i ; m\right]\right. \\
& \left.+\left(1-\frac{n}{2}\right) \chi_{s}\left[j^{-}, j ; m\right]\right] \\
\sim & \frac{1}{16 \delta^{3}}\left[\chi_{s}\left[i^{-}, i ; m\right]+\chi_{s}\left[j^{-}, j ; m\right]\right] .
\end{aligned}
$$

Notice that the derivatives of $g_{1}$ and $g_{2}$ are independent of the spin index $\sigma$. We next compute the derivatives of $\mu[i, k]$ with spin diagonal sources, by using the above equations and the derivatives of the second and third factors of Eq. (68), 


$$
\begin{aligned}
\left.\frac{\mu_{\uparrow \uparrow}(i, k)}{\delta V_{m}^{\uparrow}}\right|_{V \rightarrow 0}= & \frac{\left(1-\frac{n}{2}\right)}{2(1-n)}\left\{\chi_{t}\left[k^{-}, k ; m\right]-\chi_{t}\left[i^{-}, i ; m\right]+(1-n)\right. \\
& \left.\times\left[\chi_{s}\left[i^{-}, i ; m\right]-\chi_{s}\left[k^{-}, k ; m\right]\right]\right\} \\
\sim & \frac{1}{4 \delta}\left[\chi_{t}\left[k^{-}, k ; m\right]-\chi_{t}\left[i^{-}, i ; m\right]\right]
\end{aligned}
$$

and similarly

$$
\begin{aligned}
\left.\frac{\mu_{\uparrow \uparrow}(i, k)}{\delta V_{m}^{\downarrow \downarrow}}\right|_{V \rightarrow 0}= & \frac{\left(1-\frac{n}{2}\right)}{2(1-n)}\left\{\chi_{t}\left[i^{-}, i ; m\right]-\chi_{t}\left[k^{-}, k ; m\right]+(1-n)\right. \\
& \left.\times\left[\chi_{s}\left[i^{-}, i ; m\right]-\chi_{s}\left[k^{-}, k ; m\right]\right]\right\} \\
\sim & \frac{1}{4 \delta}\left[\chi_{t}\left[i^{-}, i ; m\right]-\chi_{t}\left[k^{-}, k ; m\right]\right] .
\end{aligned}
$$

\section{C. $\nu[i, k]$ and its derivatives}

Using the functions $g_{1} g_{2}$ in Eq. (69), We first write $\nu$ as

$$
\nu[i, k]=\nu_{1}[i, k]+\nu_{2}[i, k],
$$$$
\nu_{1}[i, k]=g_{1}[i, k] \bar{\nu}[i, k], \quad \text { and } \quad \nu_{2}[i, k]=g_{2}[i, k] \bar{\nu}[i, k] \text {, }
$$

and assuming spin diagonal sources, we find from Eq. (A10),

$$
\begin{aligned}
\bar{\nu}_{\uparrow \uparrow}[i, k]= & \left(1-\hat{G}_{\uparrow \uparrow}\left[i^{-}, i\right]\right)\left(1-\hat{G}_{\uparrow \uparrow}\left[k^{-}, k\right]\right)\left\{\hat{G}_{\uparrow \uparrow}\left[i^{-}, i\right] \chi^{(1)}\left[i^{-}, i ; k\right]\right. \\
+ & \left.\hat{G}_{\downarrow \downarrow}\left[i^{-}, i\right] \chi^{(2)}\left[i^{-}, i ; k\right]\right\}, \\
\overline{\bar{\nu}}_{\uparrow \uparrow}[i, k]= & -\left(1-\hat{G}_{\uparrow \uparrow}\left[k^{-}, k\right]\right) \chi^{(1)}\left[i^{-}, i ; k\right]-\left(1-\hat{G}_{\downarrow \downarrow}\left[k^{-}, k\right]\right) \chi^{(3)} \\
& \times\left[i^{-}, i ; k\right] .
\end{aligned}
$$

In order to obtain the leading order terms in inverse hole density, we note that the factors $1-\hat{G}$ generate factors of 1 $-n$ which lower the order of the term, unless we differentiate these terms first. Hence to leading order, the calculation is simply done by ignoring the source dependence of the $\chi$ 's in Eq. (76), and differentiating the factors of $1-\hat{G}$. We thus obtain the leading derivatives of the two objects $\nu_{1}$ and $\nu_{2}$ as follows:

$$
\begin{aligned}
\left.\frac{\delta \nu_{1 \uparrow \uparrow}[i, k]}{\delta V_{m}^{\uparrow}}\right|_{V \rightarrow 0}= & \frac{1}{16 \delta^{2}} \chi_{s}\left[i^{-}, i ; k\right]\left(\chi_{s}\left[i^{-}, i ; m\right]+\chi_{t}\left[i^{-}, i ; m\right]\right. \\
& \left.-\chi_{t}\left[k^{-}, k ; m\right]\right), \\
\left.\frac{\delta \nu_{1 \uparrow \uparrow}[i, k]}{\delta V_{m}^{\downarrow \downarrow}}\right|_{V \rightarrow 0}= & \frac{1}{16 \delta^{2}} \chi_{s}\left[i^{-}, i ; k\right]\left(\chi_{s}\left[i^{-}, i ; m\right]-\chi_{t}\left[i^{-}, i ; m\right]\right. \\
& \left.+\chi_{t}\left[k^{-}, k ; m\right]\right), \\
\frac{\delta \nu_{2 \uparrow \uparrow}[i, k]}{\left.\delta V_{m}^{\uparrow}\right|_{V \rightarrow 0}=} & \frac{1}{16 \delta^{2}}\left\{\chi_{s}\left[i^{-}, i ; k\right]\left(\chi_{t}\left[k^{-}, k ; m\right]-\chi_{s}\left[i^{-}, i ; m\right]\right)\right. \\
& \left.-\chi_{t}\left[i^{-}, i ; k\right]\left(3 \chi_{s}\left[i^{-}, i ; m\right]+\chi_{t}\left[k^{-}, k ; m\right]\right)\right\},
\end{aligned}
$$

$$
\begin{aligned}
\left.\frac{\delta \nu_{2 \uparrow \uparrow}[i, k]}{\delta V_{m}^{\downarrow \downarrow}}\right|_{V \rightarrow 0}= & \frac{1}{16 \delta^{2}}\left\{\chi_{t}\left[i^{-}, i ; k\right]\left(\chi_{t}\left[k^{-}, k ; m\right]-3 \chi_{s}\left[i^{-}, i ; m\right]\right)\right. \\
& \left.-\chi_{s}\left[i^{-}, i ; k\right]\left(\chi_{s}\left[i^{-}, i ; m\right]+\chi_{t}\left[k^{-}, k ; m\right]\right)\right\} .
\end{aligned}
$$

Using the Nozières relations we can combine these and write the final answers in the singlet and triplet channels

$$
\begin{aligned}
\left.\frac{\delta \nu[i, k]}{\delta V_{m}}\right|_{V \rightarrow 0} ^{\text {singlet }}= & -\frac{3}{8 \delta^{2}} \chi_{s}\left[i^{-}, i ; m\right] \chi_{t}\left[i^{-}, i ; k\right], \\
\left.\frac{\delta \nu[i, k]}{\delta V_{m}}\right|_{V \rightarrow 0} ^{\text {triplet }}= & \frac{1}{8 \delta^{2}}\left\{\chi_{s}\left[i^{-}, i ; k\right] \chi_{t}\left[i^{-}, i ; m\right]\right. \\
& \left.-\chi_{t}\left[i^{-}, i ; k\right] \chi_{t}\left[k^{-}, k ; m\right]\right\} .
\end{aligned}
$$

Let us now consider expressions, Eqs. (67), (74), and (77), where we find terms with $\lambda, \lambda^{2}$ explicitly appearing (where $\lambda=\frac{1}{\delta}$ ). The final answer of each term is either finite or vanishes, and to see this we must recognize the implicit dependence on $\delta$ of the coefficients. The two point susceptibilities $\chi_{s, t}\left[i, i^{-} ; j\right]$, with arbitrary arguments are seen to vanish linearly as $\delta \rightarrow 0$ since these involve a particle and a hole in the quasiparticle band, which becomes completely filled at half filling. By the same token all three-point susceptibilities also vanish linearly as $\delta \rightarrow 0$. We should also remember that the vertices themselves are nonvanishing as $\delta \rightarrow 0$. This behavior is readily confirmed by a few low-order calculations. As a useful example of this organization, we next write all the terms in the vertices that have an explicit quadratic dependence on $\lambda$. These are obtained by assembling the explicitly $O\left(\lambda^{2}\right)$ terms in Eqs. (67), (74), and (77), giving us

$$
\begin{aligned}
\Gamma_{s}[i, j ; m]= & \delta[i, m] \delta[j, m]+\frac{3}{8} \lambda^{2}\left\{t[i, j] \chi_{s}[i, i ; m] \chi_{t}[i, i ; j]\right. \\
& \left.-\delta[i, j] \frac{1}{2} J[i, k] \chi_{s}[k, k ; m] \chi_{t}[k, k ; i]\right\}, \\
\Gamma_{t}[i, j ; m]= & \delta[i, m] \delta[j, m]-\frac{1}{8} \lambda^{2} t[i, j]\left\{\chi_{s}[i, i ; j] \chi_{t}[i, i ; m]\right. \\
& \left.-\chi_{t}[i, i ; j] \chi_{t}[j, j ; m]\right\}-\frac{1}{16} \lambda^{2} \delta[i, j] J[i, k]\left\{\chi_{t}[k, k ; m]\right. \\
& \left.\times\left(3 \chi_{t}[k, k ; i]-\chi_{s}[k, k ; i]\right)-2 \chi_{t}[i, i ; m] \chi_{t}[k, k ; i]\right\} .
\end{aligned}
$$

The vertex corrections are clearly nonzero as $\delta \rightarrow 0$ since the factor $\lambda^{2}$ is compensated by two vanishing susceptibilities. These can be recognized from the detailed list in Appendix E Eqs. (G2) and (G3) as arising from $\Gamma[i, j ; m]_{2}$ and $\Gamma[i, j ; m]_{5}$ in both singlet and triplet channels. We will see next that these vertices satisfy current conservation and hence are studied further in Sec. VII E. We emphasize that these do not exhaust the set of terms that are finite as $\delta \rightarrow 0$, and constitute a convenient subset of the surviving terms. 


\section{ELECTRICAL CONDUCTIVITY, CURRENT CONSERVATION, AND WARD IDENTITIES}

We next set up the calculation of the electrical conductivity for the ECQL. We are interested in deriving a useful Kubo-type expression for conductivity in the $t$ - $J$ model, in terms of the appropriate Green's functions developed here. We will also establish Ward identities ${ }^{27-29}$ that relate the current and charge vertices, and find explicit expressions for the current vertex. These Ward identities are important relations since they constrain the possible approximations one makes, and are necessary satisfy gauge and rotation invariance of the final results. The charge and number densities and currents are trivially related by a factor of $q_{e}$ the electronic charge, and so we will work with the number densities only. The number density $n[\vec{r} \tau]=X_{\vec{r}}^{\sigma \sigma}(\tau)$ has an associated current density given by

$$
\begin{gathered}
\vec{J}(\vec{r}) \equiv \frac{1}{2} \sum_{\vec{\eta}} \vec{J}\left(r+\frac{1}{2} \vec{\eta}\right), \\
\vec{J}\left(r+\frac{1}{2} \vec{\eta}\right) \equiv i t_{\vec{\eta}} \vec{\eta}\left(X_{\vec{r}+\vec{\eta}}^{\sigma 0} X_{\vec{r}}^{0 \sigma}-X_{\vec{r}}^{\sigma 0} X_{\vec{r}+\vec{\eta}}^{0 \sigma}\right),
\end{gathered}
$$

where $\vec{\eta}$ is the set of unit vectors connecting a site to its neighbors, $\Sigma_{\sigma}$ is implied in all terms. Similar expressions can be written down also for the spin-density conservation, but since the $t$ - $J$ model involves terms that flip the spin explicitly, the form of the resulting equations differ from the charge Ward identities. ${ }^{30}$ We will be satisfied with using the Nozières relations to verify compliance with rotation invariance; these are necessary and most often sufficient conditions for giving us an invariant theory. The lattice version of divergence of these currents is written in terms of the $\mathcal{T}[\vec{r}]$ densities as

$$
\begin{gathered}
{\left[\vec{\nabla}_{\vec{r}} \cdot \vec{J}(\vec{r})\right] \equiv-i \mathcal{T}[\vec{r}]=\sum_{\vec{\eta}} \vec{\eta} \cdot \vec{J}\left(\vec{r}+\frac{1}{2} \vec{\eta}\right),} \\
\mathcal{T}(\vec{r})=\sum_{\vec{\eta} \sigma} t_{\vec{\eta}}\left(X_{\vec{r}}^{\sigma 0} X_{\vec{r}+\vec{\eta}}^{0 \sigma}-X_{\vec{r}+\vec{\eta}}^{\sigma 0} X_{\vec{r}}^{0 \sigma}\right) .
\end{gathered}
$$

The total current operator can be written

$$
\vec{J}=\sum_{r} \vec{J}(r)=i \sum_{\vec{r}, \vec{\eta}} \vec{\eta} t_{\vec{\eta}} X_{\vec{r}+\vec{\eta}}^{\sigma 0} X_{\vec{r}}^{0 \sigma}
$$

from Eq. (80), and in case of external magnetic fields, we add a suitable Peierls phase factor to the currents. This expression for $\vec{J}$ is necessary for the calculation of the frequency-dependent electrical conductivity tensor ${ }^{23,24}$ of the $t$ - $J$ model with $\Omega_{n}=2 \pi k_{B} T n$,

$$
\begin{aligned}
& \sigma_{\alpha \beta}\left(i \Omega_{n}\right)=\frac{1}{N_{S} \Omega_{n}}\left\{\left\langle\hat{\mathbf{T}}_{\alpha \beta}\right\rangle+\Pi_{\alpha \beta}\left(i \Omega_{n}\right)\right\}, \\
& \Pi_{\alpha \beta}\left(i \Omega_{n}\right)=q_{e}^{2} \int_{0}^{\beta} d \tau e^{i \Omega_{n} \tau}\left\langle T_{\tau} J^{\alpha}(\tau) J^{\beta}\right\rangle,
\end{aligned}
$$

where we set the lattice constant $a_{0}$ and $\hbar$ to unity. Here $\hat{\mathbf{T}}_{\alpha \beta}$ is the diamagnetic part of the response related to the plasma sum rule, ${ }^{31}$ and may be evaluated as an equal-time correlation. As explained in standard texts, ${ }^{23,24}$ this expression should be analytically continued as $i \Omega_{n} \rightarrow \omega+i 0^{+}$, in order to obtain the retarded physical conductivity. Our task is now to express $\Pi(i \Omega)$ in terms of the Green's functions in a manner that satisfies current conservation.

Since we are primarily interested in electromagnetic response, e.g., the optical conductivity, the condition $Q a_{0} \ll 1$ is satisfied. Hence we need long-wavelength response where the lattice structure is not crucial for the current conservation laws. However, the calculation is most effectively performed by first noticing that one can find a lattice version of the current conservation law, Eq. (85), valid at all length scales as detailed below. This more powerful conservation law ${ }^{28}$ reduces to standard electrical conservation law $\partial_{t} \rho(r, t)$ $+\nabla \cdot \vec{J}(r, t)=0$ at long wavelengths. Our strategy is to derive the exact consequences of the lattice version of the conservation law, and then to take the long-wavelength limit to obtain the electromagnetic vertices.

Using the Heisenberg equations of motion in imaginary time, it is easy to establish the exact conservation laws of number density $\partial_{\tau} n(\vec{r} \tau)=i\left[\vec{\nabla}_{\vec{r}} \cdot \vec{J}(\vec{r})\right]$ compactly in operator form

$$
\partial_{\tau} n[\vec{r} \tau]=\mathcal{T}[\vec{r} \tau]
$$

where $\mathcal{T}$ is defined above in Eq. (82). The gauge invariance of the $t-J$ Hamiltonian is ultimately responsible for these conservation laws. In order to implement the conservation laws, Eq. (85), we first establish the equation for the Green's functions as

$$
\begin{gathered}
\partial_{\tau_{m}}\left\langle\left\langle X_{i}^{0 \sigma_{i}} X_{f}^{\sigma_{f}{ }^{0}} n[m]\right\rangle\right\rangle-\left\langle\left\langle X_{i}^{0 \sigma_{i}} X_{f}^{\sigma_{f}}{ }^{0} \mathcal{T}[m]\right\rangle\right\rangle \\
=(\delta[i, m]-\delta[f, m]) \mathcal{G}_{\sigma_{i} \sigma_{f}}[i, f],
\end{gathered}
$$

where the terms on the RHS arise from the discontinuities of time ordering implied in the Green's functions. ${ }^{27}$ The two Green's functions in Eq. (86) can be obtained from the action in Eq. (13) by adding terms

$$
\mathcal{A} \rightarrow \mathcal{A}+\sum_{\vec{r}_{m}} \int_{0}^{\beta} d \tau_{m}(u[m] n[m]+v[m] \mathcal{T}[m]),
$$

where it can be seen that the effect of $u[m]$ is precisely that of $\mathcal{V}_{m}^{\sigma \sigma}$ after summing over $\sigma$ and thus gives the singlet susceptibilities and vertices. Our next task is to rewrite Eq. (86) in terms of $\hat{G}$. Toward this we recall that $\mathcal{G}[i, f]$ $=\Delta[i] \cdot \hat{G}[i, f]$, and note that

$$
\left\{\partial_{\tau_{m}} \frac{\delta}{\delta u[m]}-\frac{\delta}{\delta v[m]}\right\} \Delta[i]=0
$$

on using the conservation law, Eq. (85). Putting these together, we may thus rewrite Eq. (86) in matrix form as

$$
\left\{\partial_{\tau_{m}} \frac{\delta}{\delta u[m]}-\frac{\delta}{\delta v[m]}\right\} \hat{G}[i, f]=(\delta[i, m]-\delta[f, m]) \hat{G}[i, f],
$$




$$
\left\{\partial_{\tau_{m}} \frac{\delta}{\delta u[m]}-\frac{\delta}{\delta v[m]}\right\} \hat{G}^{-1}[i, f]=(\delta[i, m]-\delta[f, m]) \hat{G}^{-1}[i, f],
$$

where the disconnected parts cancel identically upon using the conservation law, Eq. (85). The second form is specially useful for checking the compliance of conservation laws, it is the most compact statement of the Ward identity.

We define new " $\mathcal{T}$ susceptibilities" and vertices

$$
\chi_{s}^{\mathcal{T}}[i, j ; m]=\frac{\delta}{\delta v[m]} \hat{G}[i, j], \quad \Gamma_{s}^{\mathcal{T}}[i, j ; m]=-\frac{\delta}{\delta v[m]} \hat{G}^{-1}[i, j] .
$$

The subscripts $s$ are to denote the singlet, i.e., charge nature of these currents. Below we obtain the electromagnetic vertices from these by a limiting process. These objects satisfy the real-space Ward identities

$$
\left\{\partial_{\tau_{m}} \chi_{s}[i, f ; m]-\chi_{s}^{\mathcal{T}}[i, f ; m]\right\}=(\delta[i, m]-\delta[f, m]) \hat{G}[i, f]
$$

or

$$
\left\{\partial_{\tau_{m}} \Gamma_{s}[i, f ; m]-\Gamma_{s}^{\mathcal{T}}[i, f ; m]\right\}=(\delta[f, m]-\delta[i, m]) \hat{G}^{-1}[i, f] .
$$

Fourier transforming these we obtain the momentum-space Ward identities

$$
\begin{gathered}
i\left(\omega_{p_{1}}-\omega_{p_{2}}\right) \chi_{s}\left[p_{1}, p_{2}\right]-\chi_{s}^{\mathcal{T}}\left[p_{1}, p_{2}\right]=\hat{G}\left[p_{2}\right]-\hat{G}\left[p_{1}\right], \\
i\left(\omega_{p_{1}}-\omega_{p_{2}}\right) \Gamma_{s}\left[p_{1}, p_{2}\right]-\Gamma_{s}^{\mathcal{T}}\left[p_{1}, p_{2}\right]=\hat{G}^{-1}\left[p_{1}\right]-\hat{G}^{-1}\left[p_{2}\right] .
\end{gathered}
$$

We next evaluate the $\mathcal{T}$ vertex, Eq. (89). The effect of $v[m]$ is most easily seen as a modification of the kinetic energy since the $\mathcal{T}$ operator in Eq. (82) resembles the kinetic energy closely. In fact we can write

$$
\sum_{m} v[m] \tau[m]=\sum_{m, k} t_{m, k}(v[m]-v[k]) X_{m}^{\sigma 0} X_{k}^{0 \sigma},
$$

and recalling the definition of Hamiltonian (1), we can see that the effect of adding this term in the relevant timedomain equations is to replace $t[m, k] \rightarrow t[m, k](1+v[k]$ $-v[m])$. With this observation, we may write the Green'sfunction equation, Eqs. (42) and (43), with these added sources. We again split the Green's function as in Eq. (42),

$$
\hat{G}^{-1}[i, j]=\hat{G}_{0}^{-1}[i, j]-\mathcal{S}[i, j],
$$

where

$$
\begin{aligned}
-\hat{G}_{0}^{-1}[i, j] \equiv & {\left[\partial_{\tau_{i}}-\mu+V_{i}+u[i]+\frac{1}{2} J[i, k]\left(1-\frac{n}{2}\right)\right] \delta[i, j] } \\
& -t[i, j][1+v[j]-v[i]]\left(1-\frac{n}{2}\right),
\end{aligned}
$$

and the self-energy is given by

$$
\begin{aligned}
\mathcal{S}[i, j]= & \Phi_{i} \delta[i, j]-t[i, j][1+v[j]-v[i]][\Delta[j]+\nu[i, j] \\
& \left.-\left(1-\frac{n}{2}\right)\right]-t[i, l][1+v[l]-v[i]] \Theta[i, l, j] \\
& +\frac{1}{2} J[i, k] \mu[i, k] \cdot \Theta[k, i, j]+\frac{1}{2} J[i, k][\mu[i, k] \cdot \nu[k, i] \\
& \left.+\mu[i, k] \cdot \Delta[i]-\left(1-\frac{n}{2}\right)\right] \delta[i, j] .
\end{aligned}
$$

The charge and current vertices follow from taking the derivatives of the above equation. All vertices are naturally split into bare and correction terms

$$
\Gamma=\gamma+\hat{\Gamma},
$$

where $\gamma \equiv-\frac{\delta}{\delta u} \hat{G}_{0}^{-1}$ and $\hat{\Gamma}=\frac{\delta}{\delta u} \mathcal{S}$. It follows from Eq. (94) that

$$
\gamma_{s}[i, j ; m]=\delta[i, m] \delta[j, m],
$$

$$
\gamma_{s}^{\mathcal{T}}[i, j ; m]=-t[i, j](\delta[j, m]-\delta[i, m])\left(1-\frac{n}{2}\right) .
$$

In Fourier space we find

$$
\begin{gathered}
\gamma_{s}\left[p_{1}, p_{2}\right]=1, \\
\gamma_{s}^{\mathcal{T}}\left[p_{1}, p_{2}\right]=\left(\varepsilon_{p_{1}}-\varepsilon_{p_{2}}\right)\left(1-\frac{n}{2}\right) .
\end{gathered}
$$

We observe that the bare vertices with Eq. (50) satisfy the Ward identity, Eq. (91).

In order to make contact with our calculation of the Schwinger-Dyson vertices, we consider the susceptibility

$$
\begin{aligned}
\Upsilon_{s}^{\mathcal{T}}[i, j ; m] \equiv & \frac{\delta}{\delta v[m]} \mathcal{G}_{\sigma \sigma}[i, j]=\left\langle\left\langle X_{i}^{0 \sigma} X_{j}^{\sigma 0} \mathcal{T}[m]\right\rangle\right\rangle-\left\langle\left\langle X_{i}^{0 \sigma} X_{j}^{\sigma 0}\right\rangle\right\rangle \\
& \times\langle\langle\mathcal{T}[m]\rangle\rangle, \\
\Upsilon_{s}^{\mathcal{T}}\left[p_{1}, p_{2}\right]= & \sum_{i, j, m} e^{-i p_{1}(i-m)-i p_{2}(m-j)} \Upsilon_{s}^{\mathcal{T}}[i, j ; m] \\
= & \sum_{i, j, m, \vec{\eta}, \sigma^{\prime}} e^{-i p_{1}(i-m)-i p_{2}(m-j)} t_{\vec{\eta}}\left(1-e^{i \vec{\eta} \cdot\left(\vec{p}_{1}-\vec{p}_{2}\right)}\right) \\
& \times\left\{\left\langle\left\langle X_{i}^{0 \sigma} X_{j}^{\sigma 0} X_{m}^{\sigma^{\prime} 0} X_{m+\eta}^{0 \sigma^{\prime}}\right\rangle\right\rangle-\operatorname{disc}\right\},
\end{aligned}
$$

where "disc" stands for the disconnected part and while $\sigma$ is fixed, $\sigma^{\prime}$ is summed over. In the second line onwards we turn off the sources, hence we may ignore the disc pieces due to parity. We should also note that $\eta$ is a vector without a time component, so that the two factors $X_{m} X_{m+\eta}$ are at the same time $\tau_{m}$. We next use the definition of the current from Eq. (83), and take a specific limit 


$$
\begin{aligned}
& -\frac{\partial}{\partial p^{\prime \alpha}} \Upsilon_{s}^{\mathcal{T}}\left[\vec{p} \omega_{p}, \vec{p}^{\prime} \omega_{p}+\Omega_{n}\right] /_{p^{\prime} \rightarrow p} \\
& =\sum_{\vec{r}_{i}, \vec{r}_{j}} \int_{0}^{\beta} \int_{0}^{\beta} d\left(\tau_{i}-\tau_{m}\right) d\left(\tau_{m}-\tau_{j}\right) \\
& \quad \times e^{i \omega_{p}\left(\tau_{i}-\tau_{j}\right)+i \Omega_{n}\left(\tau_{m}-\tau_{j}\right)-i \vec{p} \cdot\left(\vec{r}_{i}-\vec{r}_{j}\right)} \\
& \quad \times\left\langle\left\langle X_{i}^{0 \sigma}\left(\tau_{i}\right) X_{j}^{\sigma 0}\left(\tau_{j}\right) J^{\alpha}\left(\tau_{m}\right)\right\rangle\right\rangle .
\end{aligned}
$$

Convoluting with the velocity

$$
v_{p}^{\beta}=\frac{\partial}{\partial p^{\beta}} \varepsilon_{p}=i \sum_{\vec{\eta}} \eta^{\beta} t_{\vec{\eta}} e^{-i \vec{p} \cdot \vec{\eta}}
$$

we see that

$$
\begin{aligned}
& 2 \sum_{p} e^{i \omega_{p} 0^{+}} v_{p}^{\beta}\left(\frac{\partial}{\partial p^{\prime \alpha}} \Upsilon_{s}^{\mathcal{T}}\left[\vec{p} \omega_{p}, \vec{p}^{\prime} \omega_{p}+\Omega_{n}\right]\right)_{p^{\prime} \rightarrow p} \\
& \quad=\int_{0}^{\beta} d\left(\tau_{m}-\tau_{i}\right) e^{i \Omega_{n}\left(\tau_{m}-\tau_{i}\right)}\left\langle\left\langle J^{\alpha}\left(\tau_{m}\right) J^{\beta}\left(\tau_{i}\right)\right\rangle\right\rangle \\
& \quad=\Pi_{\alpha \beta}\left(i \Omega_{n}\right),
\end{aligned}
$$

where the factor of 2 in front is from the spin summation. We note that the response functions $Y$ can be related to the derivatives of $\hat{G}$ in a straightforward way, as in Appendix F. We obtain

$$
\begin{gathered}
\Upsilon_{s}^{\mathcal{T}}\left[p_{1}, p_{2}\right]+\Upsilon_{s}^{\mathcal{T}}\left[p_{2}-p_{1}\right] \mathcal{G}\left[p_{2}\right]=\left(1-\frac{n}{2}\right) \chi_{s}^{\mathcal{T}}\left[p_{1}, p_{2}\right] \\
\Upsilon_{s}^{\mathcal{T}}\left[p_{2}-p_{1}\right]=\left(1-\frac{n}{2}\right)^{2} \chi_{s}^{\mathcal{T}}\left[p_{2}-p_{1}\right] .
\end{gathered}
$$

For notational convenience, in the following we write $p^{\prime}$ $=p+Q$ with $Q=\left\{\vec{Q}, i \Omega_{n}\right\}$. Thus we can rewrite Eq. (101) as

$$
\begin{aligned}
\Pi_{\alpha \beta}\left(i \Omega_{n}\right)= & 2\left(1-\frac{n}{2}\right) \sum_{p} e^{i \omega_{p} 0^{+}} v_{p}^{\beta}\left(\frac { \partial } { \partial Q ^ { \alpha } } \left\{\chi_{s}^{\mathcal{T}}[p, p+Q]\right.\right. \\
& \left.\left.-\left(1-\frac{n}{2}\right) \chi_{s}^{\mathcal{T}}[Q] \hat{G}[p+Q]\right\}\right)_{\vec{Q} \rightarrow 0},
\end{aligned}
$$

where the bare current vertex $\left(1-\frac{n}{2}\right) v_{p}^{\beta}$ is a consequence of the definition of the current, Eq. (83). We make the implicit assumption that $Q$ in the vector vertex $\Gamma_{s}^{\alpha}[p, p+Q]$ always satisfies $\vec{Q} a_{0} \ll 1$, and all term of $O\left[\left(Q^{\alpha}\right)^{2}\right]$ are thrown out, i.e., we are in the electromagnetic regime. Observing from the bare vertex, Eq. (97), that $\gamma_{s}^{\mathcal{T}}[p, p+Q]$, and hence the full vertex $\Gamma_{s}^{\mathcal{T}}[p, p+Q]$ vanishes as $\vec{Q} \rightarrow 0$, and its derivative is odd under parity, we conclude that the second term in Eq. (103) can be dropped. We may then define the vector (electromagnetic) vertex (with $\vec{Q} a_{0} \ll 1$ )

$$
\begin{gathered}
\Gamma_{s}^{\alpha}[p, p+Q]=-\frac{\partial}{\partial Q^{\alpha}} \Gamma_{s}^{\mathcal{T}}[p, p+Q], \\
\chi_{s}^{\alpha}[p, p+Q]=\hat{G}[p] \Gamma_{s}^{\alpha}[p, p+Q] \hat{G}[p+Q]
\end{gathered}
$$

with the bare vector vertex $\vec{\gamma}$ as

$$
\vec{\gamma}_{s}[p, p+Q]=-\left(\frac{\partial}{\partial \vec{Q}} \gamma_{s}^{\mathcal{T}}[p, p+Q]\right)_{\vec{Q} \rightarrow 0}=\vec{v}_{p}\left(1-\frac{n}{2}\right)
$$

so that

$$
\begin{aligned}
\Pi_{\alpha \beta}(Q)= & -2 \sum_{p} e^{i \omega_{p} 0^{+}} \gamma_{s}^{\beta}[p, p+Q] \hat{G}[p] \Gamma_{s}^{\alpha}[p, p+Q] \hat{G}[p \\
& +Q] .
\end{aligned}
$$

The Ward identity, Eq. (91), relevant for electromagnetic response now reads

$$
i \Omega_{n} \Gamma_{s}[p, p+Q]-\vec{Q} \cdot \vec{\Gamma}_{s}[p, p+Q]=\hat{G}^{-1}[p+Q]-\hat{G}^{-1}[p] .
$$

In practice, this infinitesimal form of the Ward identity, Eq. (107), is more easy to implement and also sufficient for the gauge invariance of the final theory, as compared with the finite form, Eq. (91).

Returning to the Eq. (95), the equations for the vertex corrections $\hat{\Gamma}$ follow from taking derivatives of the selfenergy, Eq. (94). We may write the charge vertex simply as

$$
\begin{aligned}
\hat{\Gamma}_{S}[i, j ; m] & =\left(\frac{\delta}{\delta u[m]} \mathcal{S}_{\sigma \sigma}[i, j]\right)_{u \rightarrow 0, v \rightarrow 0} \\
& =\sum_{\sigma^{\prime}}\left(\frac{\delta}{\delta V_{m}^{\sigma^{\prime} \sigma^{\prime}}} \mathcal{S}_{\sigma \sigma}[i, j]\right)_{V \rightarrow 0}
\end{aligned}
$$

with the spin index $\sigma$ fixed at say $\uparrow$. Here the current-type sources $v[\mathrm{~m}]$ are irrelevant and could be set equal to zero right away in Eq. (94). The second identity in Eq. (108) is a consequence of the rotation invariance relations of Nozières as discussed in Appendix C.

In order to obtain the current-type vertex corrections, we get two sets of contributions from Eq. (94). We write with say a fixed $\sigma$, let us say $\sigma=\uparrow$,

$$
\begin{gathered}
\hat{\Gamma}_{s}^{\mathcal{T}}[i, j ; m]=\hat{\Gamma}_{s a}^{\mathcal{T}}[i, j ; m]+\hat{\Gamma}_{s b}^{\mathcal{T}}[i, j ; m], \\
\hat{\Gamma}_{s a}^{\mathcal{T}}[i, j ; m]=\left(\frac{\delta}{\delta v[m]} \mathcal{S}_{\sigma \sigma}^{\prime}[i, j]\right)_{u \rightarrow 0, v \rightarrow 0}, \\
\hat{\Gamma}_{s b}^{\mathcal{T}}[i, j ; m]=(\delta[i, m]-\delta[j, m]) t[i, j] \nu_{\sigma \sigma}[i, j]+(\delta[l, m] \\
-\delta[i, m]) t[i, l] \hat{G}[l, k] \Gamma^{(p)}[k, j ; i] .
\end{gathered}
$$

The set of terms $\hat{\Gamma}_{s b}^{\mathcal{T}}[i, j ; m]$ arise from the explicit factors of $v[m]$ in Eq. (94). Here $\mathcal{S}^{\prime}$ represents all the terms of Eq. (94) but with the explicit factors of $v[m]$ omitted. Thus the term $\hat{\Gamma}_{s a}^{\mathcal{T}}[i, j ; m]$ arises from differentiating the rest of the $v[m]$ dependence and originate in exactly the same terms that contribute to Eq. (108). The only difference is that after taking the derivative, a current vertex replaces the charge vertex. Therefore one can obtain the equations satisfied by $\hat{\Gamma}_{s a}^{\mathcal{T}}[i, j ; m]$ from those obeyed by $\hat{\Gamma}_{s}[i, j ; m]$, in a simple way. In the RHS, we simply replace terms indexed by the external symbol $m$, e.g., $\Gamma_{s}[p, q ; m]$ by $\Gamma_{s}^{\mathcal{T}}[p, q ; m]$ for any $p, q$. The 
same separation is also carried out for the electromagnetic vertex $\Gamma_{s}^{\alpha}$, and may be obtained by taking the limit as in Eq. (104). We note the vertices $\hat{\Gamma}_{s b}$ in Fourier space (with a fixed $\sigma)$

$$
\begin{gathered}
\hat{\Gamma}_{s b}^{\mathcal{T}}\left[p_{1}, p_{2}\right]=\sum_{q}\left(\varepsilon_{q+p_{1}}-\varepsilon_{q+p_{2}}\right) \nu_{\sigma \sigma}[q] \\
+\sum_{q}\left(\varepsilon_{q+p_{1}-p_{2}}-\varepsilon_{q}\right) G[q] \Gamma^{(p)}\left[q, p_{2}\right], \\
\vec{\Gamma}_{s b}[p, p+Q]=\sum_{q} \vec{v}_{p+q} \nu_{\sigma \sigma}[q]+\sum_{q} \vec{v}_{q} G[q] \Gamma^{(p)}[q, p+Q] .
\end{gathered}
$$

We summarize the requirements for a "conserving approximation" 14 for the ECQL, it consists of a suitable approximation for the self-energy Eqs. (51) and (54), the density vertex Eq. (108) and the current vertex Eqs. (109) and (110). These must be consistent, i.e., satisfy the Ward identity, Eq. (107), so that the response is gauge invariant.

\section{GENERAL PROPERTIES OF THE GREEN'S FUNCTION AND SUCCESSIVE APPROXIMATIONS}

We next turn to computing the Green's functions from the above theory, after making a series of approximations. Let us summarize the various objects of interest. Our interest is initially in the positive-definite spectral function $\rho_{G}[q, \nu]$, and the occupation of the $k$ state $m_{k}$ defined through the representation

$$
\hat{G}\left[k, i \omega_{k}\right]=\int_{-\infty}^{\infty} d \nu \frac{\rho_{G}[k, \nu]}{i \omega_{k}-\nu}, \quad \rho_{G}[k, \nu]=-\frac{1}{\pi} \Im m \hat{G}\left[k, \nu+i 0^{+}\right],
$$

where the positive-definite spectral density has a representation

$$
\rho_{G}[k, \nu]=\frac{1}{1-\frac{n}{2}} \sum_{\alpha \beta}\left|\left\langle\alpha\left|X^{0 \sigma}(k)\right| \beta\right\rangle\right|^{2}\left(p_{\alpha}+p_{\beta}\right) \delta\left(\nu+\varepsilon_{\beta}-\varepsilon_{\alpha}\right)
$$

with $p_{\alpha}=\frac{1}{Z_{G C}} \exp \left(-\beta \varepsilon_{\alpha}\right), \varepsilon_{\alpha}$ is an eigenvalue of the grand canonical Hamiltonian $H-\mu N$ and $Z_{G C}$ the grand canonical partition function. We will define the quasiparticle occupation number $m_{k}$ through

$$
\begin{aligned}
m_{k} & =\frac{1}{1-\frac{n}{2}}\left\langle X^{\sigma 0}(k) X^{0 \sigma}(k)\right\rangle \\
& =k_{B} T \sum_{i \omega_{k}} e^{i \omega_{k} 0^{+}} \hat{G}\left[k, i \omega_{k}\right] \\
& =\int d \nu f(\nu) \rho_{G}[k, \nu], \quad f(\nu)=\frac{1}{e^{\beta \nu}+1} .
\end{aligned}
$$

Here $\sigma$ is either up or down, and $0 \leq m_{k} \leq 1$ fixes the total number of electrons through the sum rule

$$
\frac{1}{N_{s}} \sum_{\vec{k}} m_{k}=\frac{n}{2-n}
$$

We expect that at $T=0$, a sharp Fermi surface of the ECQL exists generically, and is determined by one of several criteria in analogy with that of the Fermi liquid. Let us list them separately now. (i) The particle number sum rule, Eq. (114), is one of them. (ii) A jump in $m_{k}$ is expected at the Fermi surface so that the locus of jumps in this defines the "Migdal" version of the Fermi surface. (iii) Another is the analog of the Luttinger-Ward sum rule that says that the Fermi surface is the domain satisfying the condition $\operatorname{Re} \hat{G}(k, 0) \geq 0$, or from the spectral representation, Eq. (112), we require

$$
\sum_{\alpha \beta}\left|\left\langle\alpha\left|X^{0 \sigma}(k)\right| \beta\right\rangle\right|^{2} \frac{p_{\alpha}+p_{\beta}}{\varepsilon_{\beta}-\varepsilon_{\alpha}} \geq 0 .
$$

(iv) The final criterion requires that the quasiparticle lifetime is infinite at the FS so that the locus of points where $\rho_{\Sigma}(k, 0)=0$ defines the Fermi surface. The approximate solutions for $\hat{G}$ can be tested with these criteria, and we shall discuss only the particle number sum rule, Eq. (114), initially.

Below we also need the "bubble susceptibility" object $\chi_{0}[Q]$ defined through

$$
\chi_{0}[Q]=\sum_{q} G[q] G[q+Q]
$$

which can be evaluated in terms of the spectral functions readily as

$$
\begin{aligned}
\chi_{0}[Q]= & -\frac{1}{N_{s}} \sum_{\vec{q}} \iint d \nu_{1} d \nu_{2} \rho_{G}\left[q, \nu_{1}\right] \rho_{G}\left[q+Q, \nu_{2}\right] \\
& \times \frac{f\left(\nu_{1}\right)-f\left(\nu_{2}\right)}{\nu_{2}-\nu_{1}-i \Omega_{Q}} .
\end{aligned}
$$

We next describe a few systematic and consistent (i.e., Ward identity compliant) approximations that can be implemented. Detailed numerical calculations within these schemes are currently under way, and the results will be presented later. ${ }^{32}$

\section{A. Atomic limit}

The atomic limit is defined by switching off $t$ and $J$, and so it rather trivial. We make sure that we recover the exact answer known in this limit. $^{33,34}$ We set $\Gamma_{s}\left[p_{1}, p_{2}\right]$ $=\Gamma_{t}\left[p_{1}, p_{2}\right]=1$ and hence $\rho_{G}[k, \nu]=\delta(\nu+\mu)$, with $\hat{G}^{-1}\left[k, i \omega_{k}\right]=i \omega_{k}+\mu$, so that the chemical potential is given by

$$
f(-\mu)=\frac{n}{2-n}
$$

We compute the susceptibilities from $\chi_{s}=\chi_{t}=\chi_{0}$ with 


$$
\chi_{0}[Q]=-\beta \delta_{Q, 0} \delta_{\Omega_{Q}, 0} \frac{n(1-n)}{2\left(1-\frac{n}{2}\right)^{2}},
$$

which agrees with the sum rule in Eq. (F11).

\section{B. First approximation}

The first approximation consists of choosing $\hat{G}_{0}[k]$, Eq. (50), and the bare vertices, Eq. (97). These clearly satisfy the full set of Ward identities, In fact this approximation, with $J \rightarrow 0$ coincides with Hubbard's approximate solution, the so called Hubbard-I solution of the equations of motion ${ }^{33}$ in the limit as $U \rightarrow \infty$. The particle number can be fixed using Eq. (114), and we find that the Fermi-surface volume encloses a fraction $\frac{n}{2-n}$ of the first Brillouin zone, rather than the Luttinger-Ward fraction of $\frac{n}{2}$. The quasiparticle number is greater than the bare particle number and this feature persists in all subsequent approximations. This violates the Luttinger-Ward ${ }^{22}$ volume theorem, ${ }^{35}$ we discuss its implications in greater depth later in Sec. IX. ${ }^{36}$

The susceptibility is just the Lindhard function $\chi_{0}$ $=-\chi_{\text {Lind }}[Q]$,

$$
\chi_{\text {Lind }}[Q]=\frac{1}{N_{s}} \sum_{\vec{q}} \frac{f\left(E_{q}^{(0)}-\mu\right)-f\left(E_{q+Q}^{(0)}-\mu\right)}{E_{q+Q}^{(0)}-E_{q}^{(0)}-i \Omega_{Q}}
$$

with $E_{q}^{(0)}=\left(1-\frac{n}{2}\right) \varepsilon_{q}$. This function vanishes as we approach half filling $n \rightarrow 1$ and has the van Hove fingerprints of the above defined "large Fermi surface." It is straightforward to show that this also satisfies the sum rule, Eq. (F10), and from Eqs. (F5) and (F6), the physical spin susceptibility is $2 \mu_{B}^{2} \frac{\left(1-\frac{n}{2}\right)^{2}}{(1-n)} \chi_{L}[Q]$ and the physical charge susceptibility or compressibility is $\left(1-\frac{n}{2}\right)^{2} \chi_{L}[Q]$. Owing to the structure of the prefactors, the spin susceptibility interpolates smoothly between the Pauli and Curie susceptibilities on pushing the density toward half filling $n \rightarrow 1$, while the charge susceptibility vanishes near half filling.

\section{Hartree approximation (HA)}

The next approximation we make consists of choosing a frequency-independent vertex function and the self-energy that is also frequency independent. We write

$$
\hat{G}_{H}^{-1}[k]=\left(i \omega_{k}+\mu\right)-E_{k}^{(H)} .
$$

Specializing to only nearest-neighbor hoppings, we see from symmetry that the form of the band dispersion remains a simple tight-binding one. Therefore for simple cubic lattices using Eqs. (55)-(58) we write

$$
\begin{gathered}
\xi_{0} \equiv \frac{1}{1-\frac{n}{2}}\left\{\left(1-\frac{n}{2}\right)^{2}+\left\langle\vec{S}_{\overrightarrow{0}} \cdot \vec{S}_{\vec{\eta}}\right\rangle+\frac{1}{4}\left(\left\langle n_{0} n_{\vec{\eta}}\right\rangle-n^{2}\right)\right\}, \\
\quad \text { or } \xi_{0}=\left(1-\frac{n}{2}\right)+\frac{n}{4-2 n}\left\{3 x_{t}+(1-n) x_{s}\right\} .
\end{gathered}
$$

The vertices are taken to be the lowest consistent ones

$$
\begin{gathered}
\Gamma_{s}\left[p_{1}, p_{2}\right]=1, \quad \Gamma_{t}\left[p_{1}, p_{2}\right]=1, \\
\Gamma_{s}^{\mathcal{T}}\left[p_{1}, p_{2}\right]=\left(1-\frac{n}{2}\right)\left(\varepsilon_{p_{1}}-\varepsilon_{p_{2}}\right)+\sum_{q}\left(\varepsilon_{q+p_{1}}-\varepsilon_{q+p_{2}}\right) \nu[q] .
\end{gathered}
$$

We can check that these variables satisfy the Ward identities, Eq. (91), exactly. The object $E_{k}^{(H)}$ is frequency independent, and hence to this order, the single-particle spectral function is a simple delta function $\rho_{G}[k, \nu]=\delta\left(\nu+\mu-E_{k}^{(H)}\right)$. Thus $\hat{G}^{(H)}$ has a sharp pole with no lifetime effects. The equations are nonlinear since the $\chi$ 's are given by

$$
\chi_{H}[Q]=\frac{1}{N_{s}} \sum_{\vec{Q}} \frac{f\left(E_{q}^{(H)}-\mu\right)-f\left(E_{q+Q}^{(H)}-\mu\right)}{E_{q+Q}^{(H)}-E_{q}^{(H)}-i \Omega_{Q}},
$$

analogous to Eq. (118) but with energies $E_{k}^{(H)}$ defined below in Eq. (120). The Hartree energies contain a temperaturedependent renormalization of the bandwidth via the spinand charge-correlation functions. These nearest-neighbor charge and spin correlations determine $\xi_{0}$ and lead to a shrinking of the bandwidth in case of antiferromagnetic correlations. For ferromagnetic, i.e., Nagaoka-Thouless-type correlations, one has the opposite effect and magnetism promotes kinetic motion. Near half filling the density-dependent term in Eq. (120) is suppressed while the spin term survives. Due to antiferromagnetic correlations expected at short distances, the parameter $\xi_{0}$ is potentially smaller than unity, temperature dependent and can vanish, giving rise to a metal-insulator transition. The self-consistent solution of $\chi_{H}[q]$ determines the spin and charge correlation functions.

\section{Hartree Fock approximation (HFA)}

We next outline the Hartree Fock approximation where the Green's function contains the exchange term obtained from Eqs. (51) and (54) by setting the vertices $\Gamma_{s} \rightarrow 1$ and $\Gamma_{t} \rightarrow 1$. The bare vertices are frequency independent, and all vertex corrections are functions of frequency that vanish at high frequencies. Hence the Hartree Fock energy is the best possible frequency-independent approximation to the correct excitation energy. For this reason, it also gives the correct form of the first moment of the Green's function. We write

$$
\hat{G}_{\mathrm{HF}}^{-1}[k]=\left(i \omega_{k}+\mu\right)-E_{k}^{(\mathrm{HF})}, \quad E_{k}^{(\mathrm{HF})}=E_{k}^{(H)}-\frac{1}{2} \zeta_{\mathrm{HF}} J_{k},
$$




$$
\zeta_{\mathrm{HF}}=\frac{1}{N_{s}} \sum_{q} \cos \left(q_{x}\right) m_{q}=\left\langle X_{\bar{\eta}}^{\sigma 0} X_{0}^{0 \sigma}\right\rangle,
$$

where we dropped a $k$-independent term in the self-energy (from $\Sigma_{q} \varepsilon_{q} m_{q}$ ), and assumed the nearest-neighbor hopping on a simple cubic lattice to simplify the expressions. The vertex functions can be written down from inspection as

$$
\begin{gathered}
\Gamma_{s}\left[p_{1}, p_{2}\right]=1-\frac{1}{2} \sum_{q} J_{p_{1}-q} \chi_{s}\left[q, q+p_{2}-p_{1}\right], \\
\Gamma_{t}\left[p_{1}, p_{2}\right]=1+\frac{1}{2} \sum_{q} J_{p_{1}-q} \chi_{t}\left[q, q+p_{2}-p_{1}\right], \\
\Gamma_{s}^{\mathcal{T}}\left[p_{1}, p_{2}\right]=\left(1-\frac{n}{2}\right)\left(\varepsilon_{p_{1}}-\varepsilon_{p_{2}}\right)+\sum_{q}\left(\varepsilon_{q+p_{1}}-\varepsilon_{q+p_{2}}\right) \nu[q] \\
-\frac{1}{2} \sum_{q} J_{p_{1}-q} \chi_{s}^{\mathcal{T}}\left[q, q+p_{2}-p_{1}\right] .
\end{gathered}
$$

It is straightforward to verify that the Ward identities, Eq. (91), are satisfied exactly, and so this is a consistent scheme as well. Unlike the earlier cases discussed, this approximation generates frequency-dependent vertices. The vertices $\Gamma_{s, t}\left[p_{1}, p_{2}\right]$ are now functions of the momenta as well as the frequency difference $\omega_{p_{2}}-\omega_{p_{1}}$. The susceptibility $\chi_{\mathrm{HF}}[Q]$ can be obtained after solving the vertex functions, the singlet and triplet susceptibilities now differ from each other. The triplet susceptibility is enhanced at a finite value of $\vec{Q}$, whereas the singlet susceptibility is suppressed at finite $\vec{Q}$.

\section{E. Nonlinear Hartree (Fock) approximation [NLH(F)A]}

The next approximation is obtained from Eq. (79), where the terms of $O\left(\lambda^{2}\right)$ are isolated. Fourier transforming Eq. (79) we find

$$
\begin{gathered}
\Gamma_{s}\left[p_{1}, p_{2}\right]=1-\lambda^{2} \chi_{s}\left[p_{2}-p_{1}\right] \xi_{s}\left[p_{1}, p_{2}\right], \\
\xi_{s}\left[p_{1}, p_{2}\right]=\frac{3}{16} \chi_{\mathrm{loc}}\left\{2 \varepsilon_{p_{2}}^{t}+J_{p_{2}-p_{1}}^{t}\right\}, \\
\Gamma_{t}\left[p_{1}, p_{2}\right]=1+\lambda^{2} \chi_{t}\left[p_{2}-p_{1}\right] \xi_{t}\left[p_{1}, p_{2}\right], \\
\xi_{t}\left[p_{1}, p_{2}\right]=\frac{1}{16} \chi_{\mathrm{loc}}\left\{2 \varepsilon_{p_{2}}^{s}+J_{p_{1}-p_{2}}^{s}+2 J_{0}^{t}-3 J_{p_{1}-p_{2}}^{t}-2 \varepsilon_{p_{1}}^{t}\right\}
\end{gathered}
$$

with $\chi_{\text {loc }}$ given in Eq. $(F 11)$. The "T $\mathcal{T}$ vertex" follow from the stated rules, and we write

$$
\begin{aligned}
\Gamma_{s}^{\mathcal{T}}\left[p_{1}, p_{2}\right]= & \left(1-\frac{n}{2}\right)\left(\varepsilon_{p_{1}}-\varepsilon_{p_{2}}\right)+\sum_{q}\left(\varepsilon_{q+p_{1}}-\varepsilon_{q+p_{2}}\right) \nu[q] \\
& -\lambda^{2} \chi_{s}^{\mathcal{T}}\left[p_{2}-p_{1}\right] \xi_{s}\left[p_{1}, p_{2}\right] .
\end{aligned}
$$

The partner Green's functions of the vertices, Eq. (125), are taken from the Hartree approximation, Eq. (119). Using an important corollary of Eq. (91),

$$
i\left(\omega_{p_{1}}-\omega_{p_{2}}\right) \chi_{s}\left[p_{2}-p_{1}\right]=\chi_{s}^{\tau}\left[p_{2}-p_{1}\right],
$$

it is readily seen that the Ward identities, Eq. (91), are satisfied for these Green's functions and vertices, and thereby the NLHA is also a conserving scheme. We may add the Fock terms to the self-energy [the $\zeta$ term in Eq. (123) as well as vertices (the $J$-dependent terms on the RHS of the Eq. (124)], exactly as we did in going from the Hartree to the Hartree Fock theory. This produces the Fock generalization of the NLHA, i.e., the NLHFA. We will content ourselves with a few comments about the structure of the NLHA below.

As in the case of the Hartree Fock approximation, the vertices are now frequency dependent. We begin by multiplying Eq. (125) with $\hat{G}\left[p_{1}\right] \hat{G}\left[p_{2}\right]$ and integrating on one of the two momenta to get the susceptibilities

$$
\chi_{s, t}[Q]=\sum_{q} G[q] \Gamma_{s, t}[q, q+Q] G[q+Q] .
$$

The answer is given as

$$
\begin{aligned}
\left(\chi_{s}[Q]\right)^{-1} & =\left(\chi_{0}[Q]\right)^{-1}+\frac{3}{16} \lambda^{2} \chi_{\mathrm{loc}}\left\{J_{Q}^{t}+2 \mathbf{E}_{Q}^{t}\right\}, \\
\left(\chi_{t}[Q]\right)^{-1}= & \left(\chi_{0}[Q]\right)^{-1}-\frac{1}{16} \lambda^{2} \chi_{\mathrm{loc}}\left\{2 J_{0}^{t}-3 J_{Q}^{t}+J_{Q}^{s}\right. \\
& \left.+2\left(\mathbf{E}_{Q}^{s}-\mathbf{E}_{Q}^{t}\right)\right\}
\end{aligned}
$$

using the energy-type variables

$$
\mathbf{E}_{Q}^{s}=\frac{\sum_{q} \varepsilon_{q+Q}^{s} G[q] G[q+Q]}{\chi_{0}[Q]},
$$

and similarly for the triplet channel. A rough approximation is to ignore the frequency dependence of the energy-type variable $\mathbf{E}^{s}, \mathbf{E}^{t}$ and to think of them as further renormalized versions of the band energies and the bare exchange. We denote these energies with bold letters, to emphasize this point and to distinguish these from the previously defined energies that are all real. Let us first recall from their definitions, Eqs. (116) and (F11), that both the bubble $\chi_{0}$ and the local susceptibility $\chi_{\text {loc }}$ are negative variables. If we treat $\mathbf{E}_{Q} \propto \varepsilon_{Q}$, we see that the content of Eq. (129) is to enhance the physical susceptibility $\left(-\chi_{t}\right)$ and to decrease the physical charge compressibility $\left(-\chi_{s}\right)$, by amounts that are sensitively dependent on the prefactors. The theory has some resemblance to the random-phase approximation but with several coefficients including $x_{s}, x_{t}$ that are found self-consistently. The results will be published separately. ${ }^{32}$

\section{F. Frequency-dependent self-energy}

From the above discussion, in all the schemes discussed so far the self-energy involved in the calculations is frequency independent. This frequency dependence is important since it provides a measure of the decay of quasiparticles. We see however that the vertices are frequency independent only for the first few approximations and become frequency de- 
pendent in the HF, NLHA, and NLHFA schemes. Since the self-energy ultimately derives its frequency dependence from the vertex as in Eqs. (51) and (54), we must find a scheme where both vertices and self-energy are frequency dependent. This is an important problem to be addressed in future work. However, as a via media solution, we may take the frequency-dependent vertices and substitute them into Eq. (54), as a nonself-consistent first approximation. To lowest order we find terms that are reminiscent of those encountered in Fermi liquids with $\mathcal{S} \propto G G G$, i.e., with the convolution of a bubble susceptibility with a Green's function. This integral is a familiar one from Fermi-liquid theory, and the imaginary part of the self-energy is generically $\propto \omega^{2}$. The FS volume is unchanged from the Hartree value in a manner that is quite similar to the standard FL theory. There are several detailed question that remain to be worked out regarding the shrinking of the bandwidth and of its temperature dependence, we will return to these in future ${ }^{32}$

\section{ANISOTROPIC $d$-WAVE PAIRING AND SUPERCONDUCTING INSTABILITY OF THE ECQL}

We next study the possibility of a superconducting instability of the ECQL. We study the $t-J$ model without any added terms such as phonons, and hence we are looking at the possibility of a spontaneous instability of the type that ${ }^{3} \mathrm{He}$ undergoes, when it becomes a superfluid. ${ }^{37-39}$

Near half filling, a full Gorkov-Nambu-type calculation with anomalous Green's functions within this formalism is feasible for this purpose at the level of the NLHA, and we will present the details later. ${ }^{32}$ To find the existence of a $d$-wave instability, we can take a shortcut; following the precedent in ${ }^{3} \mathrm{He}$, we extract the effective interaction from the NLHA vertices, Eq. (125). Onsite $s$-wave pairing is excluded by the prohibition of double occupancy, and is automatic in the full Gorkov-Nambu-type scheme for the ECQL, within our formalism. ${ }^{32}$ However, in the present phenomenological scheme, we must implement it by ignoring all but the $d$-wave channel. ${ }^{40}$

The singlet and triplet vertices in Eq. (125) are at the (NL) Hartree level, and from these we can extract the irreducible interactions ${ }^{26}{ }^{1} I\left(p, p^{\prime} ; Q\right)$ and ${ }^{0} I\left(p, p^{\prime} ; Q\right)$ in the two channels, and using crossing symmetry deduce the pairing interaction in the particle-particle channel. As Leggett points out, ${ }^{38}$ this is achieved more transparently when we fit these interactions to a pseudopotential $U_{p p}$ treated at the same (Hartree) level, ${ }^{41}$ and then consider the pairing of the pseudopotential Hamiltonian $H=H_{0}+H_{p p}^{\prime}$. A strong shortranged repulsion is added to eliminate $s$-wave channel, this is necessary since we consider singlet pairing unlike ${ }^{3} \mathrm{He}$, where the triplet pairing forbids the $s$-wave channel, by symmetry. The total pseudopotential then is $U_{p p}^{\prime}(1,2)=(\infty)$ $\times \delta(1,2)+U_{p p}(1,2)$, and the (spin-dependent) rotationally invariant pseudopotential given by ${ }^{41}$

$$
\begin{gathered}
U_{p p}(1,2)=V(1,2)+\vec{\sigma}_{1} \cdot \vec{\sigma}_{2} W(1,2), \\
H_{p p}^{\prime}=\frac{1}{2} \sum_{p_{1}+p_{2}=p_{3}+p_{4}}\left\langle p_{1} \sigma_{1}, p_{2} \sigma_{2}\left|U_{p p}^{\prime}\right| p_{3} \sigma_{3}, p_{4} \sigma_{4}\right\rangle \hat{c}_{p_{1} \sigma_{1}}^{\dagger} \\
\times \hat{c}_{p_{2} \sigma_{2}}^{\dagger} \hat{c}_{p_{4} \sigma_{4}} \hat{c}_{p_{3} \sigma_{3}}
\end{gathered}
$$

$$
\begin{aligned}
& \left\langle p_{1} \sigma_{1}, p_{2} \sigma_{2}\left|U_{p p}\right| p_{3} \sigma_{3}, p_{4} \sigma_{4}\right\rangle \\
& =\delta_{\sigma_{1} \sigma_{3}} \delta_{\sigma_{2} \sigma_{4}}\left\{\left\langle p_{1}, p_{2}|V| p_{3}, p_{4}\right\rangle+\sigma_{1} \sigma_{2}\left\langle p_{1}, p_{2}|W| p_{3}, p_{4}\right\rangle\right\} \\
& \quad+\delta_{\sigma_{1} \bar{\sigma}_{3}} \delta_{\sigma_{2} \bar{\sigma}_{4}} \delta_{\sigma_{1} \bar{\sigma}_{2}}\left\langle p_{1}, p_{2}|W| p_{3}, p_{4}\right\rangle
\end{aligned}
$$

where $\hat{c}$ are regarded as the Hartree quasiparticles, and we proceed to find the vertex correction for this Hamiltonian at the Hartree level

$$
\begin{gathered}
\Gamma_{s}\left[p_{1}, p_{2}\right]=1+2 \sum_{p_{1}+p_{2}^{\prime}=p_{2}+p_{1}^{\prime}}\left\langle p_{2} p_{1}^{\prime}|V| p_{1} p_{2}^{\prime}\right\rangle \chi_{s}\left[p_{1}^{\prime}, p_{2}^{\prime}\right], \\
\Gamma_{t}\left[p_{1}, p_{2}\right]=1+2 \sum_{p_{1}+p_{2}^{\prime}=p_{2}+p_{1}^{\prime}}\left\langle p_{2} p_{1}^{\prime}|W| p_{1} p_{2}^{\prime}\right\rangle \chi_{t}\left[p_{1}^{\prime}, p_{2}^{\prime}\right],
\end{gathered}
$$

where $\chi_{s}\left[p_{1}^{\prime}, p_{2}^{\prime}\right]=G\left[p_{1}^{\prime}\right] \Gamma_{s}\left[p_{1}^{\prime}, p_{2}^{\prime}\right] G\left[p_{2}^{\prime}\right]$, etc. Comparing with Eq. (125) we see that

$$
\begin{aligned}
& 2\left\langle p_{2}, p_{1}^{\prime}|V| p_{1}, p_{2}^{\prime}\right\rangle=-\lambda^{2} \xi_{s}\left[p_{1}, p_{2}\right], \\
& 2\left\langle p_{2}, p_{1}^{\prime}|W| p_{1}, p_{2}^{\prime}\right\rangle=+\lambda^{2} \xi_{t}\left[p_{1}, p_{2}\right] .
\end{aligned}
$$

We can now insert these potentials into the standard anisotropic Cooper-pairing problem. ${ }^{39,41}$ For the case of singlet pairing, we require the pseudopotential for the process $\mid p_{2} \uparrow$ $\left.-p_{2} \downarrow\right\rangle \rightarrow\left|p_{1} \uparrow-p_{1} \downarrow\right\rangle$. We write the required matrix element of $V-W$,

$$
\begin{aligned}
U_{p p}\left(p_{1}, p_{2}\right)= & \frac{1}{32} \frac{n}{(1-n)\left(1-\frac{n}{2}\right)^{2}}\left\{2 J_{0} x_{t}+\left(x_{s}+2 x_{t}\right)\left(\varepsilon_{p_{1}}+\varepsilon_{p_{2}}\right)\right. \\
& \left.+x_{s} J_{p_{1}-p_{2}}\right\} .
\end{aligned}
$$

In obtaining this expression, we have used the definitions of $\xi_{s}$ and $\xi_{t}$ in Eq. (125), and symmetrized these in $p_{1}, p_{2} \cdot{ }^{20}$ It is easy to see that only the third term survives in the $d$-wave channel, and thus we truncate further

$$
U_{p p}^{d \text { wave }}\left(p_{1}, p_{2}\right)=\frac{1}{32} \frac{n}{(1-n)\left(1-\frac{n}{2}\right)^{2}} x_{S} J_{p_{1}-p_{2}} .
$$

Clearly the same expression holds for the full pseudopotential $U^{\prime}$ since the strong repulsion has $s$-wave-type symmetry.

The term $x_{t} J_{p_{1}-p_{2}}$ cancels out in taking $\xi_{s}+\xi_{t}$ above, and we observe that the exchange energy $J_{p_{1}-p_{2}}$ is multiplied by the nearest-neighbor density-density correlations function $x_{s}=\left(\left\langle n_{\vec{r}_{i}} n_{\vec{r}_{i}+\vec{\eta}}\right\rangle-n^{2}\right) /\left(n-n^{2}\right)$ defined in Eq. (56). This object is closely connected to the pair-distribution function discussed in the electron gas, and is well known to have a correlation hole, i.e., particles avoid getting close to each other regardless of their spin. In the $t-J$ model, a similar depletion is expected so that we expect $x_{s}<0$. If we consider a fully spin-polarized liquid, then we can compute $x_{s}$ easily from a Fermi gas picture, and we find $x_{s}=-\frac{1}{n-n^{2}}\left|\left\langle c_{0}^{\dagger} c_{\vec{\eta}}\right\rangle\right|^{2}$. This object is negative and small near half filling $\propto-(1-n)$. We expect $x_{s}$ to be negative in the ECQL in the paramagnetic limit, although the magnitude should be larger than that for the fer- 
romagnet, since particles need to be neighbors in order to benefit from the exchange interaction.

We now treat $U_{p p}^{d \text { wave }}$ within the pairing scheme ${ }^{41}$ and write down the gap equation for $d$-wave singlet superconductivity

$$
\begin{gathered}
\Delta\left(p_{1}\right)=-\sum_{p_{2}} U_{p p}^{d \text { wave }}\left(p_{1}, p_{2}\right) \rho\left(p_{2}\right) \Delta\left(p_{2}\right), \\
\rho(p)=\left\{\frac{\tanh \left[\frac{1}{2} \beta E(p)\right]}{2 E(p)}\right\},
\end{gathered}
$$

where $E(p)=\left[\xi_{p}^{2}+|\Delta(p)|^{2}\right]^{1 / 2}$, with the Hartree energies $\xi_{p}$ $=\xi_{0}\left(\varepsilon_{k}-\mu_{0}\right)$, with $\mu_{0}$ the chemical potential from the first approximation (without the $\xi_{0}$ correction), and

$$
\xi_{0}=\frac{1}{1-\frac{n}{2}}\left[\left(1-\frac{n}{2}\right)^{2}+\left\langle\vec{S}_{i} \cdot \vec{S}_{i+\eta}\right\rangle+\frac{1}{4}\left(\left\langle n_{i} n_{i+\eta}\right\rangle-n^{2}\right)\right]
$$

as defined in the NL Hartree theory, Eq. (120). This equation can be linearized near the transition temperature by setting $E(p) \rightarrow\left|\xi_{p}\right|$ in the summand of the above equation. We assume $\Delta(p) \propto \cos \left(p_{x}\right)-\cos \left(p_{y}\right)$, and a simple analysis gives the condition for the transition temperature $T_{c}$ as

$$
\sum_{p}\left(\cos p_{x}-\cos p_{y}\right)^{2} \frac{\tanh \left[\frac{\xi_{0}}{2 k_{B} T_{c}}\left(\varepsilon_{p}-\mu_{0}\right)\right]}{2 \xi_{0}\left(\varepsilon_{p}-\mu_{0}\right)}=\frac{1}{\alpha J}
$$

or

$$
\int_{-W}^{W} d \varepsilon \rho(\varepsilon) \psi(\varepsilon) \frac{\tanh \left[\frac{\xi_{0}}{2 k_{B} T_{c}}\left(\varepsilon_{p}-\mu_{0}\right)\right]}{2 \xi_{0}\left(\varepsilon_{p}-\mu_{0}\right)}=\frac{1}{\alpha J},
$$

where

$$
\psi(\varepsilon)=\frac{1}{\rho(\varepsilon)} \sum_{p}\left(\cos p_{x}-\cos p_{y}\right)^{2} \delta\left(\varepsilon_{p}-\varepsilon\right)
$$

and

$$
\alpha=\frac{n}{32(1-n)\left(1-\frac{n}{2}\right)^{2}}\left(-x_{s}\right) \text {. }
$$

The density of states $\rho(\varepsilon)$ and the angular average $\psi(\varepsilon)$ are easily found for the square lattice in terms of the convenient variable $u=\frac{\varepsilon}{W}$ with $W=4 t$, and the elliptic integrals $E(m)$ and $K(m)$ (where $m$ is the parameter of the elliptic integrals)

$$
\rho(\varepsilon)=\frac{1}{2 \pi^{2} t} K\left(1-u^{2}\right),
$$

$$
\psi(\varepsilon)==8\left\{\frac{1}{2}\left(1+u^{2}\right)-\frac{E\left(1-u^{2}\right)}{K\left(1-u^{2}\right)}\right\} .
$$

At low temperatures, the sum diverges logarithmically from the region $\varepsilon \sim \mu_{0}$. We can extract the divergence by expanding the integrand around $\mu_{0}$, which may be safely taken to its zero-temperature limit. We thus find

$$
\frac{1}{\xi_{0}} \rho\left(\mu_{0}\right) \psi\left(\mu_{0}\right) \log \left[1.13 \beta_{c} \xi_{0}\left(W^{2}-\mu_{0}^{2}\right)^{1 / 2}\right]=\frac{1}{\alpha J},
$$

and hence

$$
k_{B} T_{c} \sim 1.13 \xi_{0} \sqrt{\left(W^{2}-\mu_{0}^{2}\right)} e^{-\xi_{0} / \xi^{*}}
$$

where

$$
\xi^{*}=\alpha J \rho\left(\mu_{0}\right) \psi\left(\mu_{0}\right) .
$$

This expression is valid provided the resulting $T_{c}$ is much smaller than the bandwidth $2 W$, and further we need the positivity of the two variables $\xi_{0}$ and $-x_{s}$. The maximum $T_{c}$ this approach can yield is $k_{B} T_{\text {max }} \sim 1.13 \xi^{*} \sqrt{\left(W^{2}-\mu_{0}^{2}\right)}$. Taking standard values for parameters in high- $T_{c}$ systems, namely, $t=6000^{\circ}$ and $J=1500^{\circ}$, this maximum $T_{c}$ decreases from $105^{\circ} \mathrm{K}$ at $n=0.75-43^{\circ} \mathrm{K}$ at $n=0.9$, provided we use the Hartree estimates for $x_{s}$ and with $x_{t}=-0.44 n$ to fit the known ground-state energy of the Heisenberg antiferromagnet. However the $T_{c}$ from Eq. (142) is much smaller than these values because $\xi_{0} / \xi^{*} \gg 1$ in the entire range.

It is interesting to compare our pairing Eqs. (136), (138), and (142) with corresponding equations in the work of Baskaran, Zou, and Anderson (BZA), ${ }^{42}$ who first proposed that the superexchange interaction could lead to superconductivity in the $t-J$ model, and to the work of Kotliar ${ }^{43}$ who generalized BZA to the case of $d$-wave symmetry. BZA's pairing equation is obtained from an intuitive argument where the exchange energy is written in a particular factorized way. Its mean-field theory results in a pairing Hamiltonian that has a striking resemblance to our pairing term $U_{p p}^{d \text { wave }}$. In fact their mean-field theory transforms to precisely to the above equations if we make the following mappings from our calculation: $\xi_{0} \rightarrow(1-n), \alpha \rightarrow 1$ and finally adjust the chemical potential $\mu_{0} \rightarrow \mu_{1}$ with $\mu_{1}$ chosen so that $\Sigma_{p} f\left(\varepsilon_{p}-\mu_{1}\right)=\frac{n}{2}$.

On the one hand the qualitative conclusions of the two approaches are very close. Within our theory, at least within the NL Hartree approximation, superconductivity is possible in the $d$-wave channel thanks to the sign of the correlator $x_{s}$; it turns exchange into an attractive interaction from its initially repulsive character. The mean-field theory of BZA obtains the attraction by a specific factorization of the exchange energy, and while it is not clear that this factorization is unique, it is consistent with the NLHA.

On the other hand, BZA attain a much greater $T_{c}$ than our calculation does. This can be tracked down to one slightly unfavorable and one crippling difference. The ratio $\xi /(1$ $-n)$ is $O(1)$ and does not make any difference, however the ratio $\rho\left(\mu_{1}\right) \psi\left(\mu_{1}\right) / \rho\left(\mu_{0}\right) \psi\left(\mu_{0}\right)$ is $O(3)$ for most of the range of densities, and this enhances their $T_{c}$ somewhat. The ratio $1 / \alpha$ is very large $\sim O(30)$ for most densities, and this makes 
our $T_{c}$ come out very small. We thus see that the dimensionless constants in our expression, Eq. (139) (e.g., the factor of 32) make all the difference between the two approaches. Our approach systematically leads to these constants as stated, at least within the NLHA, and cannot be ignored. We thus feel that superconductivity within the ECQL is very subtle, its currently precarious scale could well be influenced by correction terms beyond the NLHA considered here, and must await further investigations.

\section{PHYSICAL INTERPRETATION OF THE QUASIPARTICLES}

We next discuss the physical significance of the quasiparticles, defined as the poles of $\hat{G}$, contrasted with the bare particles obtained from $\mathcal{G}$. The number density of particles $n=N / N_{s}$ is found by taking the translationally invariant limit of

$$
\begin{aligned}
n[i]=\sum_{\sigma}\left\langle X_{i}^{\sigma \sigma}\right\rangle & =\operatorname{tr} \mathcal{G}\left[i^{-}, i\right]=\frac{1}{\gamma[i]}\left\{\operatorname{tr} \hat{G}\left[i^{-}, i\right]-2 \operatorname{det} \hat{G}\left[i^{-}, i\right]\right\} \\
& =\frac{2 \hat{G}\left[i^{-}, i\right]}{1+\hat{G}\left[i^{-}, i\right]} \quad \text { when } V \rightarrow 0 .
\end{aligned}
$$

Inverting it, we express the local quasiparticle density $n_{\mathrm{QP}}$,

$$
\begin{gathered}
n_{\mathrm{QP}}[i] \equiv \operatorname{tr} G_{\sigma \sigma}\left[i^{-}, i\right] \\
\rightarrow \frac{n}{1-\frac{n}{2}},
\end{gathered}
$$

where we have taken the paramagnetic and uniform limit in the last line. Thus the QP density is always larger than the bare density by a factor that is unity at very low fillings and approaches 2 near half (bare) filling. In the case of a general spin population with the partial densities denoted by $n_{\sigma}$ $=N_{\sigma} / N_{s}$, it is easy to see that

$$
n_{\mathrm{QP}, \sigma}=\frac{n_{\sigma}}{1-n_{\bar{\sigma}}},
$$

illustrating the fact that both spin populations of the bare electron contribute to that of the quasiparticles of a given spin. We see that the quasiparticle densities come closer to the bare ones as we polarize the $t$ - $J$ model. This is natural since the Pauli principle already keeps like spins apart so that the effect of the projection operators is reduced. In the fully polarized sector the problem reduces to that of a spinless ideal Fermi gas.

Fourier transforms can be performed on turning off the sources, since translation invariance is restored, and we can construct the occupation in momentum space [see Eq. (113)] as for the standard Fermi liquid. This is carried out in detail in Eq. (114). In the present case, it is clear from Eqs. (114) and (147) that the number of electrons "contained" in $\hat{G}$ are greater than those in $\mathcal{G}$ by a factor $\frac{1}{1-\frac{n}{2}}$. This is the "renormalized" particle number sum rule mentioned in Eq. (114). From this relation we expect that the other criteria for determining the Fermi surface outlined above [after Eq. (114)] are similarly scaled. Detailed calculations within various possible schemes are underway, and we will comment here on the basis of simple calculations. Within the Hartree or the Hartree Fock approximations, the self-energy is real and the various criteria give the same result. The analog of the Luttinger-Ward's FS volume theorem ${ }^{22}$ for the ECQL holds, provided we replace the electron density by an enhanced value as in Eq. (147). Thus we predict Fermi-surface volume $\Omega^{\mathrm{FS}}$ for the ECQL state, in comparison to the FL state and the ferromagnetic (FM) state (or equivalently spinless particles) to be given by

$$
\begin{gathered}
\Omega_{\mathrm{ECQL}}^{\mathrm{FS}}=\frac{n}{2-n}, \quad \Omega_{\mathrm{FL}}^{\mathrm{FS}}=\frac{n}{2}, \quad \Omega_{\mathrm{FM}}^{\mathrm{FS}}=n, \\
\xi^{*}=\frac{\Omega_{\mathrm{ECQL}}^{\mathrm{FS}}}{\Omega_{\mathrm{FL}}^{\mathrm{FS}}}=\frac{2}{2-n} .
\end{gathered}
$$

This renormalization of the volume by $\xi^{*}$ signifies a lack of adiabatic continuity with the noninteracting electron problem, ${ }^{44}$ a key feature of the FL. In Appendix D, we locate the origin of the breakdown of continuity. At least within the limited setting of the atomic limit of the Hubbard model, we can trace the origin of this change in volume. We study the change in functional dependence of the Green's function and self-energy upon cranking up the interaction strength $U$ at a fixed frequency $\omega_{n}$. The distinction between two highfrequency (hf) limits: weakly correlated $\left(\omega \rightarrow \infty\right.$ and $\left.\frac{U}{\omega} \rightarrow 0\right)$ or the extremely correlated $\left(\omega \rightarrow \infty\right.$ and $\left.\frac{U}{\omega} \rightarrow \infty\right)$ is responsible for the changed volume of the Fermi surface. With the insight gleaned from this exercise, we conjecture the behavior of the general Hubbard model self-energy in the limit of EC. Assuming this conjectured behavior, we provide a variation in the standard arguments ${ }^{22,23}$ that yields the renormalized quasiparticle FS volume as in Eq. (148) for the Hubbard model in the $t$ - $J$ regime of parameters. These volumes satisfy the bound

$$
\Omega_{\mathrm{FM}}^{\mathrm{FS}} \geq \Omega_{\mathrm{ECQL}}^{\mathrm{FS}} \geq \Omega_{\mathrm{FL}}^{\mathrm{FS}}
$$

so that the ECQL Fermi volume differs from both the standard cases for general filling, and approaches that of the FL and the FM states at low $(n \sim 0)$ and high densities $(n \sim 1)$, respectively.

Independently of our proposal, unbiased numerical methods have recently suggested that the Fermi volume of the two-dimensional (2D) $t$ - $J$ model differs from that of the FL by different enlargement factors $\xi^{*}$ as in Eq. (148), although 
the factors seem a bit smaller. ${ }^{45}$ One curious consequence follows for the nearest-neighbor hopping bipartite lattices, e.g., the $2 \mathrm{D}$ square lattice or the three-dimensional square lattice. At precisely $n=\frac{2}{3}$, the quasiparticle density is exactly one half. Thus the QP FS volume is half of the first Brillouin zone, and hence they occupy the nested diamond-shaped region expected for bare electrons at half filling. Beyond this filling, the curvature of the FS changes from electronlike to holelike. Therefore one would expect the Hall constant of the $t$ - $J$ model to change sign and become holelike at $n=\frac{2}{3}$. Studies of the $t-J$ model Hall constant ${ }^{46}$ are consistent with this expectation, showing a change in sign at exactly this filling.

It is instructive to deduce from the quasiparticle Green's function the time-dependent number density $n_{\mathrm{QP}}[i]$ $=\operatorname{tr} G_{\sigma \sigma}\left[i^{-}, i\right]$ and the spin density $\vec{S}_{\mathrm{QP}}[i]=\frac{1}{2} \operatorname{tr} \vec{\tau} \hat{G}\left[i^{-}, i\right]$, in terms of the bare number density $n[i]$, Eq. (144), and bare spin density $\vec{S}[i]=\frac{1}{2} \operatorname{tr} \vec{\tau} \mathcal{G}\left[i^{-}, i\right]$ with $\vec{\tau}$ the three Pauli matrices. These follow from the inverse relation

$$
\hat{G}\left[i^{-}, i\right]=\frac{1}{\operatorname{det}\left(\mathbb{1}-\mathcal{G}\left[i^{-}, i\right]\right)}\left(\mathbb{1}-\mathcal{G}\left[i^{-}, i\right]\right) \cdot \mathcal{G}\left[i^{-}, i\right],
$$

and hence

$$
\begin{aligned}
& n_{\mathrm{QP}}[i]=2-\frac{(1-n[i])(2-n[i])}{\left(1-\frac{1}{2} n[i]\right)^{2}-\vec{S}[i] \cdot \vec{S}[i]}, \\
& \vec{S}_{\mathrm{QP}}[i]=\vec{S}[i] \frac{(1-n[i])(2-n[i])}{\left(1-\frac{1}{2} n[i]\right)^{2}-\vec{S}[i] \cdot \vec{S}[i]} .
\end{aligned}
$$

Thus the quasiparticle number density is locally related to the bare particle number density and the bare spin density in a nonlinear fashion. These relations are easily inverted as well,

$$
\begin{gathered}
n[i]=\frac{2\left\{2+\left[1-n_{\mathrm{QP}}[i]\right] n_{\mathrm{QP}}[i]\right\}-\sqrt{\left(2-n_{\mathrm{QP}}[i]\right)\left\{2-n_{\mathrm{QP}}[i]-\left[2+n_{\mathrm{QP}}[i]\right]\left|\vec{S}_{\mathrm{QP}}[i]\right|^{2}\right\}}}{4-\left[n_{\mathrm{QP}}[i]\right]^{2}}, \\
\vec{S}[i]=\vec{S}_{\mathrm{QP}}[i] \frac{1}{2-n_{\mathrm{QP}}[i]} .
\end{gathered}
$$

The inverse relations are in some sense more fundamental since the quasiparticles are the basic objects that drive the bare particle response. We observe that at a given number density $n[i]$, a tendency to form a local moment by the bare particles, i.e., $|\vec{S}[i]| \rightarrow 1 / 2 n[i]$, enhances (reduces) the quasiparticle spin (number) density. Further we see that the quasiparticle spin density is scaled down from the true spin density by a factor $\sim \delta=1-n$ corresponding to the hole density measured from half filling, and also the quasiparticle band becomes full when the bare particle band becomes half filled.

It follows from Eq. (151) that the delicately structured relationship between quasiparticle charge and spin density should be best seen when we dope the uniform ECQL with a charge or spin impurity. In this case the Friedel trapping of a single bare charge ends up capturing a nontrivial (densitydependent) number of quasiparticles, and the spin density reflects the charge density as well. This leads us to expect that the role of the impurities would be important in revealing the nature of the quasiparticles. One interesting feature is that when the bare particle density is close to unity at any point, the quasiparticle spin density has a local minimum at that point, and thus displays a nonmonotonic behavior.

From the above construction, we conclude that the charge of the quasiparticles $q_{\mathrm{QP}}$ must be regarded as a densitydependent fraction of the bare charge $q_{e}$,

$$
q_{\mathrm{QP}}=\left\{1-\frac{1}{2} n\right\} q_{e},
$$

in order that the total charge remain invariant, i.e., $q_{\mathrm{QP}} N_{\mathrm{QP}}$ $=q_{e} N$. Near half filling, the charge of the quasiparticles is $\sim \frac{1}{2}$. Therefore the flow of a bare particle is equivalent to that of a sufficient number $\left(\frac{2}{2-n}\right)$ of quasiparticles so that the total charge is balanced. These fractionally charged particles are defined in the many-body context without any specific single-particle basis. These fundamentally arise in terms of a modified Pauli principle implied in Eqs. (114), (146), and (147).

\section{CONCLUSIONS AND SUMMARY}

In this work, we have presented a systematic study of the $t-J$ model by using the Schwinger technique of source fields. In addition we have developed a specific methodology to overcome the problem of noncanonical fermions forced upon us by the infinite- $U$ constraint in the model. ${ }^{47,48}$ Since the method is technically quite involved, we have presented the details in a self-contained fashion. We obtained the exact Schwinger-Dyson equation for the $t-J$ model, and hence a closed expression for the inverse Green's function $\hat{G}^{-1}[k]$ in terms of the vertex functions. Both singlet and triplet particle-hole vertices are needed to complete the definition of 
the $\hat{G}^{-1}[k]$. The vertices are reported up to the neglect of the source derivatives of the vertex, this is a natural stopping point since we need to first understand the consequences of the many terms generated so far. In order to facilitate concrete approximations, we presented the Ward identities for the current and density vertices.

The resulting Green's functions and vertices form a hierarchy; this is in many respects similar to the one usually encountered for standard Fermi systems, but with extra features that arise from the dynamical analogs of Gutzwiller's projection operators ${ }^{10}$ for the ground state. As with standard models of weakly interacting fermions, such a hierarchy is the proper setting for exploring other features that might lead to controlled approximations. The existence of a low-density nuclear matter (Brueckner, Galitskii, and Migdal)-type or high-density electron-gas (Bohm, Pines, Brueckner, and Gell-Mann)-type approximations in the Fermi systems are examples of such an emergent process, and are described in various texts. ${ }^{23,24,26,44}$ Our preliminary search shows a natural ordering of terms in the vertex, where the hole density plays a central role. This scheme is currently under numerical study and results will be reported later. ${ }^{32}$

The statistical mechanical equilibrium state underlying our Green's functions is the extremely correlated electron liquid. This quantum liquid breaks no spatial or temporal symmetries. It has specific signatures that distinguish it from the Fermi liquid. In particular, the Fermi-surface volume naturally differs from that of the Fermi liquid. The elementary excitations of this liquid are best viewed as fractionally charged quasiparticles, whose charge is determined by the density. At all densities of the $t-J$ model, the particle number sum rule requires a Fermi surface that is larger than the standard Luttinger-Ward Fermi surface, ${ }^{22}$ by an amount $\xi^{*}=\frac{1}{1-\frac{n}{2}}$. In Appendix D, we have presented a suggestive variation in the standard argument for the Fermi-surface volume that gives us the scaled volume. Further studies are needed to compute the spectral functions and thereby answer the issue of sharpness of the quasiparticles in a complete fashion by computing $\hat{G}^{-1}[k]$ within a controlled scheme. The fractionally charged quasiparticle picture of the ECQL is a quantitative description of the so-called lower Hubbard band. ${ }^{49}$

An interesting feature of the theory is that the $\hat{G}^{-1}[k]$ involves the static density and spin correlations at nearestneighbor separation; this leads to the narrowing of the bands and the possibility of a metal-insulator transition near half filling driven by local antiferromagnetic correlations (rather than true antiferromagnetic order). In general the ECQL is prone to various instabilities such as the antiferromagnetic, insulating, or superconducting states. We presented a calculation of the superconducting instability toward $d$-wave order in Sec. VIII, the final formulas have considerable similarity to the resonating valence-bond theory ${ }^{42}$ but end up with a much lower $T_{c}$ due to some dimensionless factors that are unavoidable in this theory. More generally, in the ECQL, unlike the standard Fermi-liquid instabilities, one does not need to deal with a large energy scale such as $U$, since the Hubbard operators already deal with the local constraint efficiently. This is a great advantage since in the FL, the energy scale $U$ skews the picture of instabilities by overemphasizing the magnetic instabilities.

\section{ACKNOWLEDGMENTS}

This work was supported by NSF under Grant No. DMR 0706128 and DOE-BES under Grant No. FG02-06ER46319.

\section{APPENDIX A: IDENTITIES INVOLVING THE SOURCE DERIVATIVES}

Let us next work out the transformation of the derivative

$$
\begin{aligned}
\Delta^{-1}[i] \cdot D[j] \cdot \Delta[k]= & \Delta^{-1}[i] \cdot \overline{D[j] \cdot \Delta[k]} \\
& +\vdots \Delta^{-1}[i] \cdot D[j] \cdot \Delta[k] \vdots,
\end{aligned}
$$

where the first term consists of $D[j]$, the overline symbol is analogous to a contraction in field theory. In the present context it implies that $D$ is acting as both a matrix as well as a derivative on $\Delta[k]$. In the second term the vertical dots denotes "normal ordering" w.r.t. the derivative operator, i.e., in matrix element form, we take the derivative operators to the right of the $\Delta[k]$. These two terms arise from the action of $D$ as a matrix derivative acting upon the $\Delta[k]$ term, and also on whatever stands to the right of the expression, and is the analog of the covariant derivative in non-Abelian gauge theory. We will next show that this may be expressed as a useful identity,

$$
\begin{aligned}
\Delta^{-1}[i] \cdot D[j] \cdot \Delta[k]= & \mu[i, j] \cdot \nu[j, k] \\
& +\vdots \mu[i, j] \cdot \mathcal{D}[j] \cdot \mu[j, k] \vdots,
\end{aligned}
$$

where $\mathcal{D}[j]$ is defined below in Eq. (A3), it is the transformed version of $D$ in terms of the transformed source term $V$. Let us first prove an identity for the derivative.

$$
\begin{gathered}
\vdots \Delta^{-1}[i] \cdot D[i] \cdot \Delta[i] \vdots=\mathcal{D}[i], \\
\mathcal{D}_{\sigma_{1}, \sigma_{2}}[i]=\sigma_{1} \sigma_{2} \frac{\delta}{\delta V_{i}^{\bar{\sigma}_{1} \bar{\sigma}_{2}}} .
\end{gathered}
$$

This useful identity implies that in calculations where the source $\mathcal{U}$ is turned off, as, e.g., in Appendix $\mathrm{F}$ where we calculate the susceptibility relations, we can ignore the distinction between $D$ and $\mathcal{D}$ since $\Delta \rightarrow 1$.

We use Eq. (24), i.e., $\Delta^{-1}[i]=\frac{1}{\operatorname{det} \Delta[i]} \Delta^{k}[i]$ to rewrite the inverse $\Delta$. Taking components, we write this equation as

$$
\begin{aligned}
& \left(\vdots \Delta^{-1}[i] \cdot D[i] \cdot \Delta[i] \vdots\right)_{\sigma_{1}, \sigma_{2}} \\
& \quad=\frac{1}{\operatorname{det} \Delta[i]} \sigma_{a} \sigma_{b} \Delta_{\sigma_{1} \sigma_{a}}^{k}[i] \Delta_{\sigma_{b} \sigma_{2}}[i] \frac{\delta}{\delta \mathcal{V}_{i}^{\bar{\sigma}_{a} \bar{\sigma}_{b}}} .
\end{aligned}
$$

Since $V_{i}=\Delta^{-1}[i] \cdot \mathcal{V}_{i} \cdot \Delta[i]$, we may write

$$
\begin{aligned}
\frac{\delta}{\delta \mathcal{V}_{i}^{\bar{\sigma}_{a}, \bar{\sigma}_{b}}} & =\frac{1}{\operatorname{det} \Delta[i]} \Delta_{\bar{\sigma}_{c} \bar{\sigma}_{a}}^{k}[i] \Delta_{\bar{\sigma}_{b} \bar{\sigma}_{d}}[i] \frac{\delta}{\delta V_{i}^{\bar{\sigma}_{c} \bar{\sigma}_{d}}} \\
& =\frac{1}{\operatorname{det} \Delta[i]} \Delta_{\sigma_{a} \sigma_{c}}[i] \Delta_{\sigma_{d} \sigma_{b}}^{k}[i] \frac{\delta}{\delta V_{i}^{\bar{\sigma}_{c}, \bar{\sigma}_{d}}},
\end{aligned}
$$

where we have using the definition of the conjugate in going 
to the second line. Substituting into Eq. (A4) we see that the spin components are now properly arranged to yield delta functions so that the identity, Eq. (A3), is proved.

From the identity, Eq. (A3), we may write another useful transformation

$$
\vdots D[i] \vdots=\vdots \Delta[i] \cdot \mathcal{D}[i] \cdot \Delta^{-1}[i] \vdots,
$$

this helps us to replace functional derivatives w.r.t. the original source $\mathcal{V}$ with those w.r.t. the transformed source $V$.

Using this, we may at once rewrite the LHS of Eq. (A2),

$$
\begin{aligned}
& \vdots \Delta^{-1}[i] \cdot D[j] \cdot \Delta[k] \vdots \\
& \quad=\vdots \Delta^{-1}[i] \cdot \Delta[j] \cdot \mathcal{D}[j] \cdot \Delta^{-1}[j] \cdot \Delta[k] \vdots \\
& \quad \vdots \mu[i, j] \cdot \mathcal{D}[j] \cdot \mu[j, k] \vdots
\end{aligned}
$$

This proves the second term in Eq. (A2).

\section{1. $\nu$ matrix}

We turn to the first term in Eq. (A2), we see from Eq. (61) that

$$
\Delta[k]=\frac{1}{\gamma[k]}\left(1-\hat{G}^{k}\left[k^{-}, k\right]\right),
$$

where we denote $\gamma[k]=1-\operatorname{det} \hat{G}\left[k^{-}, k\right]$, therefore

$$
\begin{aligned}
\nu[k, j] & =\Delta^{-1}[j] \cdot \overline{D[j] \cdot \Delta[k]} \\
& =\Delta^{-1}[j] \cdot \overline{\vdots \Delta[j] \cdot \mathcal{D}[j] \cdot \Delta^{-1}[j] \vdots \cdot \Delta[k]} \\
& =\overline{\vdots \mathcal{D}[j] \cdot \Delta^{-1}[j] \vdots \cdot \Delta[k]} .
\end{aligned}
$$

In component form we write,

$$
\begin{aligned}
\nu_{\sigma_{1}, \sigma_{2}}[k, j]= & \Delta_{\sigma_{a}, \sigma_{b}}^{-1}[j] \overline{\mathcal{D}_{\sigma_{1}, \sigma_{a}}[j] \Delta_{\sigma_{b}, \sigma_{2}}[k]} \\
= & \frac{\sigma_{1} \sigma_{a}}{\gamma[k]} \Delta_{\sigma_{a}, \sigma_{b}}^{-1}[j]\left\{\Delta_{\sigma_{b}, \sigma_{2}}[k] \hat{G}_{\sigma_{q}, \sigma_{p}}^{k}\left[k^{-}, k\right] \chi_{\bar{\sigma}_{1}, \bar{\sigma}_{a}, \sigma_{q}}^{\sigma_{a}}[k, k ; j]\right. \\
& -\sigma_{2} \sigma_{b} \chi_{\bar{\sigma}_{1}, \bar{\sigma}_{a}}^{\left.\bar{\sigma}_{2}, \bar{\sigma}_{b}[k, k ; j]\right\},}
\end{aligned}
$$

where we used

$$
\frac{\delta}{\delta V_{j}^{\sigma_{a}, \sigma_{b}}} \operatorname{det} \hat{G}\left[k^{-}, k\right]=\chi_{\sigma_{a}, \sigma_{b}}^{\sigma_{p} \sigma_{q}}[k, k ; j] \hat{G}_{\sigma_{q}, \sigma_{p}}^{k}\left[k^{-}, k\right] .
$$

For spin diagonal sources, we find

$$
\begin{aligned}
\nu_{\sigma \sigma}[k, j]= & \frac{1}{\gamma[k]}\left[\Delta _ { \sigma \sigma } ^ { - 1 } [ j ] \left\{\left[\Delta_{\sigma \sigma}[k] \hat{G}_{\sigma \sigma}[k, k]-1\right] \chi^{(1)}[k, k, j]\right.\right. \\
& \left.\left.+\Delta_{\sigma \sigma}[k] \hat{G}_{\bar{\sigma} \bar{\sigma}}\right] k, k\right] \chi^{(2)}[k, k, j]-\Delta_{\bar{\sigma} \bar{\sigma}}^{-1}[j] \chi^{(3)}[k, k, j] .
\end{aligned}
$$

Upon turning off the sources, we find

$$
\begin{aligned}
\nu_{\sigma_{1}, \sigma_{2}}[a, b]= & \delta_{\sigma_{1}, \sigma_{2}} \frac{1-\frac{n}{2}}{1-n}\left[\left(\frac{n}{2}-1\right) \chi^{(1)}[a, a ; b]+\frac{n}{2} \chi^{(2)}[a, a ; b]\right. \\
& \left.-\chi^{(3)}[a, a ; b]\right] .
\end{aligned}
$$

\section{2. $\Theta$ matrix}

We calculate the object

$$
\Theta[r, s, m]=\overline{\vdots \mathcal{D}[r] \cdot \mu[r, s] \vdots \cdot \hat{G}[s, f]} \cdot \hat{G}^{-1}[f, m],
$$

this appears above. Taking the matrix element of this we write

$$
\begin{aligned}
\Theta_{\sigma_{1}, \sigma_{2}}[r, s, m] & =\mu_{\sigma_{a}, \sigma_{b}}[r, s]\left(\mathcal{D}_{\sigma_{1}, \sigma_{a}}[r] \hat{G}_{\sigma_{b}, \sigma_{3}}[s, f]\right) \hat{G}_{\sigma_{3} \sigma_{2}}^{-1}[f, m] \\
& =\sigma_{1} \sigma_{a} \mu_{\sigma_{a}, \sigma_{b}}[r, s] \chi_{\bar{\sigma}_{1}, \sigma_{a}, \sigma_{3}}^{\sigma_{a}}[s, f ; r] \hat{G}_{\sigma_{3} \sigma_{2}}^{-1}[f, m] \\
& =\sigma_{1} \sigma_{a} \mu_{\sigma_{a}, \sigma_{b}}[r, s] \hat{G}_{\sigma_{b}, \sigma_{c}}[s, p] \Gamma_{\bar{\sigma}_{1}, \sigma_{a}}^{\sigma_{c}, \sigma_{2}}[p, m ; r] .
\end{aligned}
$$

For spin diagonal sources we find

$$
\begin{aligned}
\Theta_{\sigma, \sigma}[r, s, m]= & \Delta_{\sigma \sigma}[s] \Delta_{\sigma \sigma}^{-1}[r] \hat{G}_{\sigma \sigma}[s, k] \Gamma^{(2)}[k, m ; r] \\
& -\Delta_{\bar{\sigma} \bar{\sigma}}[s] \Delta_{\bar{\sigma} \bar{\sigma}}^{-1}[r] \hat{G}_{\bar{\sigma} \bar{\sigma}}[s, k] \Gamma^{(3)}[k, m ; r] .
\end{aligned}
$$

Upon turning off the sources, this becomes

$$
\begin{aligned}
\Theta_{\sigma_{1}, \sigma_{2}}[r, s, m] & \rightarrow \delta_{\sigma_{1}, \sigma_{2}} G[s, k]\left\{\Gamma^{(2)}[k, m ; r]-\Gamma^{(3)}[k, m ; r]\right\} \\
& =\delta_{\sigma_{1}, \sigma_{2}} G[s, k] \Gamma^{(p)}[k, m ; r]
\end{aligned}
$$

\section{APPENDIX B: THE $\Phi_{i}$ MATRIX}

We study the properties of the matrix $\Phi_{i}$ defined in Eq. (32) as

$$
\Phi_{i}=\Delta^{-1}[i] \cdot \partial_{\tau_{i}} \Delta[i],
$$

and begin by starting from Eq. (20),

$$
\begin{aligned}
\partial_{\tau_{i}} T\left[e^{-\mathcal{A}} X_{i}^{\sigma_{a}, \sigma_{b}}\left(\tau_{i}\right)\right]= & -T\left\{e^{-\mathcal{A}}\left[X_{i}^{\sigma_{a}, \sigma_{b}}\left(\tau_{i}\right), H\right]\right\} \\
& +\mathcal{V}_{1}^{\sigma_{1} \sigma_{2}}\left(\tau_{i}\right) T\left\{e^{-\mathcal{A}}\left[X_{i}^{\sigma_{1} \sigma_{2}}\left(\tau_{i}\right), X_{i}^{\sigma_{a}, \sigma_{b}}\left(\tau_{i}\right)\right]\right\}
\end{aligned}
$$

and hence

$$
\begin{aligned}
\partial_{\tau_{i}}\left\langle\left\langle X_{i}^{\sigma_{a} \sigma_{b}}\left(\tau_{i}\right)\right\rangle\right\rangle= & \mathcal{V}_{i}^{\sigma_{c} \sigma_{a}}\left(\tau_{i}\right)\left\langle\left\langle X_{i}^{\sigma_{c} \sigma_{b}}\left(\tau_{i}\right)\right\rangle\right\rangle-\mathcal{V}_{i}^{\sigma_{b} \sigma_{c}}\left(\tau_{i}\right) \\
& \times\left\langle\left\langle X_{i}^{\sigma_{a} \sigma_{c}}\left(\tau_{i}\right)\right\rangle\right\rangle+t_{i, l}\left(\left\langle\left\langle X_{i}^{\sigma_{a} 0}\left(\tau_{i}\right) X_{l}^{0 \sigma_{b}}\left(\tau_{i}\right)\right\rangle\right\rangle\right. \\
& \left.-\left\langle\left\langle X_{l}^{\sigma_{a} 0}\left(\tau_{i}\right) X_{i}^{0 \sigma_{b}}\left(\tau_{i}\right)\right\rangle\right\rangle\right) \\
& +\frac{1}{2} J_{i, l}\left(\left\langle\left\langle X_{l}^{\sigma_{a} \sigma_{c}}\left(\tau_{i}\right) X_{i}^{\sigma_{c} \sigma_{b}}\left(\tau_{i}\right)\right\rangle\right\rangle\right. \\
& \left.-\left\langle\left\langle X_{i}^{\sigma_{a} \sigma_{c}}\left(\tau_{i}\right) X_{l}^{\sigma_{c} \sigma_{b}}\left(\tau_{i}\right)\right\rangle\right\rangle\right) .
\end{aligned}
$$

Thus we obtain on using the definition $\Delta_{\sigma_{1}, \sigma_{2}}[i]=\delta_{\sigma_{1}, \sigma_{2}}$ $-\left(\sigma_{1} \sigma_{2}\right)\left\langle\left\langle X_{i}^{\bar{\sigma}_{1} \bar{\sigma}_{2}}\left(\tau_{i}\right)\right\rangle\right\rangle$, and the $k$ conjugate of the source $\left(\mathcal{V}_{i}^{(k)}\right)^{\sigma_{a}, \sigma_{b}}=\sigma_{a} \sigma_{b} \mathcal{V}_{i}^{\bar{\sigma}_{b}, \bar{\sigma}_{a}}$,

$$
\begin{aligned}
\partial_{\tau_{i}} \Delta_{\sigma_{1}, \sigma_{2}}[i]= & \left(\mathcal{G}^{(k)}[i, i] \cdot \mathcal{V}_{i}^{(k)}-\mathcal{V}_{i}^{(k)} \cdot \mathcal{G}^{(k)}[i, i]\right)-t_{i, l}\left(\sigma_{1} \sigma_{2}\right) \\
& \times\left(\left\langle\left\langle X_{i}^{\bar{\sigma}_{1} 0}\left(\tau_{i}\right) X_{l}^{0 \bar{\sigma}_{2}}\left(\tau_{i}\right)\right\rangle\right\rangle-\left\langle\left\langle X_{l}^{\bar{\sigma}_{1} 0}\left(\tau_{i}\right) X_{i}^{0 \bar{\sigma}_{2}}\left(\tau_{i}\right)\right\rangle\right\rangle\right) \\
& -\frac{1}{2} J_{i, l}\left(\sigma_{1} \sigma_{2}\right)\left(\left\langle\left\langle X_{l}^{\bar{\sigma}_{1} \bar{\sigma}_{c}}\left(\tau_{i}\right) X_{i}^{\bar{\sigma}_{c} \bar{\sigma}_{2}}\left(\tau_{i}\right)\right\rangle\right\rangle\right.
\end{aligned}
$$




$$
\left.-\left\langle\left\langle X_{i}^{\bar{\sigma}_{1} \bar{\sigma}_{c}}\left(\tau_{i}\right) X_{l}^{\bar{\sigma}_{c} \bar{\sigma}_{2}}\left(\tau_{i}\right)\right\rangle\right\rangle\right),
$$

and after some calculation we find

$$
\begin{gathered}
\Phi_{i}=V_{i}^{(k)}-\mathcal{V}_{i}^{(k)}+\bar{\Phi}_{i}, \\
\bar{\Phi}_{i}=t[i, l] \Delta^{-1}[i] \cdot\left(\mathcal{G}^{(k)}[i, l]-\mathcal{G}^{(k)}[l, i]\right)+\frac{1}{2} J[i, l](\Delta[l] \\
-\mu[i, l] \cdot \Delta[i])+\frac{1}{2} J[i, l] \Delta^{-1}[i] \cdot \overline{(D[i] \cdot \Delta[l]} \\
-\overline{D[l] \cdot \Delta[i]) .}
\end{gathered}
$$

The second and third lines of Eq. (B3) correspond to the term $\bar{\Phi}$ in Eq. (B5), and it should be noted that the time arguments of the $X$ 's are all equal. From this it is clear that the term $\bar{\Phi}$ vanishes on turning off the sources, due to the cancellation of terms obtained by interchanging $i \leftrightarrow l .^{50}$

\section{APPENDIX C: ROTATION INVARIANCE AND THE NOZIÈRES RELATIONS}

We summarize the rotational invariance argument for the various vertex functions, these are in close parallel to the standard Fermi-liquid arguments as related by Nozières. ${ }^{51}$ We further assume that time reversal and parity are also preserved in this putative ECQL state. Let us consider the case of an extremely correlated quantum liquid with no broken symmetries. Since there is no source term, rotation invariance leads to a useful spin decomposition of the vertices. A general four-legged object $\chi_{\sigma_{c} \sigma_{d}}^{\sigma_{a}, \sigma_{b}}$ is analyzed initially, and we show that other objects have the same decomposition. Since the symmetries of this liquid are identical to those of the Fermi liquid, we can extract the decomposition by studying the parallel problem. We schematically view this object as the scattering amplitude for a particle and a hole, from the definition of this object, it is related to the correlator (assuming a suitable set of particle times)

$$
\begin{aligned}
\chi_{\sigma_{c} \sigma_{d}}^{\sigma_{a} \sigma_{b}}[p, q ; r] & \equiv \frac{\delta \hat{G}_{\sigma_{a} \sigma_{b}}[p, q]}{\delta V_{r}^{\sigma_{c} \sigma_{d}}} \\
& \sim\left\langle f_{\sigma_{b}}^{\dagger}[q] f_{\sigma_{a}}[p] f_{\sigma_{c}}^{\dagger}[r] f_{\sigma_{d}}[r]\right\rangle \\
& \sim\left(\sigma_{a} \sigma_{c}\right)\left\langle f_{\sigma_{b}}^{\dagger}[q] h_{\bar{\sigma}_{a}}^{\dagger}[p] h_{\bar{\sigma}_{c}}[r] f_{\sigma_{d}}[r]\right\rangle,
\end{aligned}
$$

where $f_{\sigma}^{\dagger}$ creates a particle with spin $\sigma$, and $h_{\sigma}^{\dagger}=f_{\bar{\sigma}} \sigma$ creates a hole with a spin $\sigma$. The hole creation operator has by standard convention an extra factor of $\sigma$, in order to ensure that the spin-flip operator of the particle $f_{\uparrow}^{\dagger} f_{\downarrow}$ maps to the spin flip of the hole $h_{\uparrow}^{\dagger} h_{\uparrow}$, rather than its negative. This object is Fourier transformed according to the standard rules, Eq. (H1), and we will omit the momentum labels for brevity.

We may thus view the $\chi$ as a scattering amplitude for a process taking a particle-hole pair with a certain initial state to a final state ${ }^{52}$ as follows:

$$
\text { Initial state }=\left\{\sigma_{d}, \bar{\sigma}_{c}\right\},
$$

$$
\text { Final state }=\left\{\sigma_{b}, \bar{\sigma}_{a}\right\} .
$$

We first note that due to time-reversal invariance, the object satisfies

$$
\chi_{\sigma_{c} \sigma_{d}}^{\sigma_{a} \sigma_{b}}=\chi_{\bar{\sigma}_{c}}^{\bar{\sigma}_{a} \bar{\sigma}_{b}}
$$

with reversed momenta. However, using parity, we can reverse all momenta again restoring them to their original values, therefore this relation is true with fixed momenta.

Let us call $\vec{S}^{t o t}$ as the total spin of the particle-hole pair. We must conserve the $z$ component of this object

$$
S_{z}^{t o t}=\frac{1}{2}\left(\bar{\sigma}_{a}+\sigma_{b}\right)=\frac{1}{2}\left(\sigma_{d}+\bar{\sigma}_{c}\right) .
$$

We must also conserve the total spin magnitude $\left|\vec{S}^{t o t}\right|=0$ or 1 in this process since the ground state is rotationally a singlet. For implementing this, we further decompose the scattering into the particle-hole singlet and triplet channels as follows. The particle-hole states can be represented in one of two possible schemes that are illustrated for two of the states as follows:

\section{Scheme A}

Scheme B

Singlet $\quad\{\uparrow \downarrow-\downarrow \uparrow\} \quad\left\{f_{\uparrow}^{\dagger} h_{\downarrow}^{\dagger}-f_{\downarrow}^{\dagger} h_{\uparrow}^{\dagger}\right\} \quad\left\{f_{\uparrow}^{\dagger} f_{\uparrow}+f_{\downarrow}^{\dagger} f_{\downarrow}\right\} \quad[\uparrow \uparrow+\downarrow \downarrow]$.

Triplet $\quad\{\uparrow \downarrow+\downarrow \uparrow\} \quad\left\{f_{\uparrow}^{\dagger} h_{\downarrow}^{\dagger}+f_{\downarrow}^{\dagger} h_{\uparrow}^{\dagger}\right\} \quad\left\{f_{\uparrow}^{\dagger} f_{\uparrow}-f_{\downarrow}^{\dagger} f_{\downarrow}\right\} \quad[\uparrow \uparrow-\downarrow \downarrow]$

Using Scheme A, we write the four possible states (displaying the initial state) and the corresponding scattering amplitudes as

$$
\begin{array}{ccc} 
& \text { Singlet channel } \\
\frac{1}{\sqrt{2}}\{\uparrow \downarrow+\downarrow \uparrow\} & \chi_{s}=\frac{1}{2} \sum_{\sigma \sigma^{\prime}} \chi_{\sigma^{\prime} \sigma^{\prime}}^{\sigma \sigma} \rightarrow & \chi^{(1)}+\chi^{(2)} \\
& \text { Triplet channel } \\
\frac{1}{\sqrt{2}}\{\uparrow \downarrow-\downarrow \uparrow\} & \chi_{t}=\frac{1}{2} \sum_{\sigma \sigma^{\prime}} \sigma \sigma \chi_{\sigma^{\prime} \sigma^{\prime}}^{\sigma \sigma} \rightarrow & \chi^{(1)}-\chi^{(2)} \\
\{\uparrow \uparrow\} o r\{\downarrow \downarrow\} & \chi_{t}=\chi_{\sigma \bar{\sigma}}^{\sigma \bar{\sigma}} \rightarrow & \chi^{(3)}
\end{array}
$$

where the nonzero amplitudes are denoted as

$$
\begin{gathered}
\chi_{\sigma \sigma}^{\sigma \sigma} \equiv \chi^{(1)}=\frac{1}{2}\left(\chi_{s}+\chi_{t}\right), \\
\chi_{\bar{\sigma} \bar{\sigma}}^{\sigma \sigma} \equiv \chi^{(2)}=\frac{1}{2}\left(\chi_{s}-\chi_{t}\right), \\
\chi_{\sigma \bar{\sigma}}^{\sigma \bar{\sigma}} \equiv \chi^{(3)}=\chi_{t} .
\end{gathered}
$$

Assuming spin isotropy of the quantum liquid phase, and all states of a given spin must give the same results for the scattering amplitude. From the two possible states of the triplet channel, we glean an important and labor-saving identity, 


$$
\chi^{(3)}=\chi^{(1)}-\chi^{(2)}
$$

We find in the equations above that a specific combination for the susceptibility $\chi$ and the vertex $\Gamma$ arises repeatedly,

$$
\begin{aligned}
\chi^{(p)} & =\chi_{\bar{\sigma} \bar{\sigma}}^{\sigma \sigma}-\chi_{\bar{\sigma} \sigma}^{\bar{\sigma} \sigma}=\chi^{(2)}-\chi^{(3)} \\
& =\frac{1}{2}\left\{\chi_{s}-3 \chi_{t}\right\} .
\end{aligned}
$$

This corresponds to the correlator of singlet Cooper pairs of particles, i.e., $\left\langle b^{\dagger}[p, q] b[r, s]\right\rangle$, with $b^{\dagger}[p, q]=f_{\uparrow}^{\dagger}(p) f_{\uparrow}^{\dagger}(q)$ $-f_{\uparrow}^{\dagger}(p) f_{\uparrow}^{\dagger}(q)$, so we call it the pair channel.

We also see that the vertex function $\Gamma$ "inherits" this symmetry, Eq. (C7), of $\chi$. This follows from the definition in Eq. (40) and the isotropy and diagonal nature in spin indices of the $\hat{G}$ 's, i.e., $\hat{G}_{\sigma_{i} \sigma_{j}} \rightarrow \delta_{\sigma_{i}, \sigma_{j}} \hat{G}_{\sigma_{i}}$ on switching off the source terms.

\section{APPENDIX D: THE HUBBARD MODEL ATOMIC LIMIT AND FS VOLUME}

In this appendix, we locate the point where adiabatic continuity is lost, in going from the Hubbard model to the $t$ - $J$ model. The context of the discussion is the atomic limit, where we can trace this quite explicitly. However, the generality of the argument regarding the high-frequency limit and the asymptotic form of the Green's function and selfenergy in the two models is to be noted. One of the consequences is that we have identified the essential problem in applying the Luttinger-Ward theorem to the $t$ - $J$ model, it must suffer corrections due to the neglect of the boundary term $n_{2}$ as outlined below in Eq. (D9).

We recall the solution of the Hubbard model in the atomic limit $^{33} t \rightarrow 0$ where

$$
G_{\text {atomic }}\left(i \omega_{n}\right)=\frac{1-\frac{n}{2}}{i \omega_{n}+\mu}+\frac{\frac{n}{2}}{i \omega_{n}+\mu-U}
$$

or

$$
=\frac{1}{i \omega_{n}+\mu-\Sigma\left(i \omega_{n}\right)}
$$

with

$$
\Sigma\left(i \omega_{n}\right)=U \frac{n}{2}+U^{2} \frac{\frac{n}{2}\left(1-\frac{n}{2}\right)}{i \omega_{n}+\mu-U\left(1-\frac{n}{2}\right)} .
$$

Here the symbols $G$ and $\Sigma$ stand for the standard definitions of the Green's function and self-energy ${ }^{23,26}$ and should not be confused with the $t-J$ model objects defined in this work. ${ }^{53}$ We are interested in understanding how these functions evolve, as we go from weakly correlated (WC) to EC limits. In the EC limit we recover the $t$ - $J$ model solution, of course in the trivial limit of $t, J \rightarrow 0$. For this we need to understand two distinct hf limits

$$
\begin{gathered}
\text { WChf limit } \quad \omega_{n} \rightarrow \infty \quad U \sim O(1) \quad \frac{U}{\omega_{n}} \rightarrow 0, \\
\text { EChf limit } \quad \omega_{n} \rightarrow \infty \quad U \rightarrow \infty \quad \frac{U}{\omega_{n}} \rightarrow \infty
\end{gathered}
$$

We observe that in the WChf limit, $G \sim 1 / i \omega_{n}$ and $\Sigma \sim c 1$ $+c 2 / i \omega_{n}$, and thus behave exactly as one expects. ${ }^{23,26}$ The canonical nature of the anticommutation relations of the fermions fixes the coefficient of $1 / i \omega_{n}$ in $G$ as unity. In the EC limit, we send $U \rightarrow \infty$ first, so that

$$
G_{\text {atomic EC }}\left(i \omega_{n}\right)=\frac{1-\frac{n}{2}}{i \omega_{n}+\mu},
$$

the coefficient of $1 / i \omega_{n}$ is now $1-\frac{n}{2}$ expected behavior from Eq. (46), this reflects the noncanonical nature of the electrons in this limit. This coefficient is a statement of the density dependence of the number of states of the "lower Hubbard band." The self-energy in the EC limit, Eq. (D2), is easily found to be

$$
\Sigma_{\mathrm{EC}}\left(i \omega_{n}\right)=c_{0}\left(i \omega_{n}+\mu\right), \quad \text { where } c_{0}=-\frac{n}{2-n} .
$$

Reflection shows that this feature of a linearly growing $\Sigma$ as a function of $i \omega_{n}$, an initially surprising result, is natural in this case. A linear growth of $\Sigma$ with $i \omega_{n}$ with the exact coefficient $c_{0}$ in Eq. (D4), is needed to match the asymptotic high-frequency behavior of $G$ in Eq. (D3) with the correct and related coefficient. Thus we see that the loss of continuity in functional form between weakly correlated and extremely correlated Green's function $G$ 's and $\Sigma$, arises due of the order of limits implied by Eq. (D2). The EC limit corresponds to taking $U \rightarrow \infty$ before any other limit; this process tosses out a fraction of the states of the Hilbert space, and thereby redefines the asymptotics of the remaining scales. We conjecture that this behavior of the Hubbard model selfenergy $\Sigma$ is inevitable in the generic case of finite hopping as well. Thus our conjecture is that in the extreme correlations limit of the Hubbard model, the self-energy must have a form $\left(z=i \omega_{n}\right)$,

$$
\Sigma(\vec{k}, z) / \lim _{U \rightarrow \infty}=c_{0}(z+\mu)+\Sigma_{\text {Regular }}(\vec{k}, z)
$$

with $c_{0}$ given in Eq. (D4), where $\Sigma_{\text {Regular }}(\vec{k}, z)$ is a wellbehaved function (i.e., $\sim c_{1}+c_{2} / z$ ) at high $z$. For consistency, it must in fact be related to the self-energy $\mathcal{S}\left[\vec{k}, i \omega_{n}\right]$ of the $t$ - $J$ model in Eq. (54) with $J_{i j} \rightarrow 0$ through the relation

$$
\Sigma_{\text {Regular }}\left(\vec{k}, i \omega_{n}\right)=\frac{1}{1-\frac{n}{2}} \mathcal{S}\left[\vec{k}, i \omega_{n}\right] .
$$

Armed with the conjectured Eq. (D5), and another assumption detailed below, we can modify the original argument of Luttinger and Ward ${ }^{22}$ to obtain the volume of the ECQL Fermi surface. Following the masters, we begin with the relation between the number of particles and the Green's function (with $\eta=0^{+}$) in a FL, 


$$
\begin{aligned}
n & =2 \sum_{k} G\left(\vec{k}, i \omega_{n}\right) e^{i \omega_{n} \eta} \\
& =\frac{2}{N_{s}} \sum_{\vec{k}} \int_{-\infty}^{0} \frac{d x}{2 \pi i}\{G(\vec{k}, x-i \eta)-G(\vec{k}, x+i \eta)\} .
\end{aligned}
$$

In the first line, the frequency sum is replaced by a contour integral after multiplying by the Fermi function. Next we deform the contour to run parallel to the real line, and taking the $T=0$ limit gives the second expression. From the definition $G(\vec{k}, z)=1 /\left[z+\mu-\varepsilon_{k}-\Sigma(\vec{k}, z)\right]$, we write

$$
G(\vec{k}, z)=-\frac{d}{d z} \log G(\vec{k}, z)+G(\vec{k}, z) \frac{d}{d z} \Sigma(\vec{k}, z),
$$

so that $n=n_{1}+n_{2}$ with

$$
\begin{aligned}
n_{1}= & -\frac{2}{N_{s}} \sum_{\vec{k}} \int_{-\infty}^{0} \frac{d x}{2 \pi i}\left[\frac{d}{d x} \log G(\vec{k}, x-i \eta)\right. \\
& \left.-\frac{d}{d x} \log G(\vec{k}, x+i \eta)\right] \\
= & \frac{1}{2 \pi i} \frac{2}{N_{s}} \sum_{\vec{k}} \log \frac{G(\vec{k},-\infty-i \eta) G(\vec{k}, i \eta)}{G(\vec{k},-\infty+i \eta) G(\vec{k},-i \eta)} \\
= & \frac{2}{N_{s}} \sum_{\vec{k}} \Theta[G(\vec{k}, 0)],
\end{aligned}
$$

where $\Theta(x)$ is the usual Heaviside function ( 1 or 0 ). This evaluation is parallel to the one in the original argument, wherein $n_{1}$ is the sole contribution to the volume theorem. The number $n_{1}$, and hence in a FL the total density $n$, is found by adding up the number per volume of $k$ values where the $G(\vec{k}, 0)$ at the chemical potential is positive. We next consider

$$
\begin{aligned}
n_{2}= & \frac{2}{N_{s}} \sum_{\vec{k}} \int_{-\infty}^{0} \frac{d x}{2 \pi i}\left\{G(\vec{k}, x-i \eta) \frac{d}{d x} \Sigma(\vec{k}, x-i \eta)\right. \\
& \left.-G(\vec{k}, x+i \eta) \frac{d}{d x} \Sigma(\vec{k}, x+i \eta)\right\} .
\end{aligned}
$$

This term is usually integrated by parts ${ }^{22,23}$ and the boundary term $\Sigma(\vec{k}, x) G(\vec{k}, x)$ discarded at infinity, assuming that the growth of $\Sigma(\vec{k}, x)$ is slower than linear in $x$. From our discussion above, this assumption is incorrect for the EC or $t$-J limit. There the linearly growing $\Sigma(\vec{k}, x)$ precisely catches up with the inverse linearly decaying $G(\vec{k}, x)$, giving a nontrivial boundary contribution. The corrected answer is most easily found if we use our conjecture Eq. (D5) for decomposing $\Sigma$ into two parts, and we find

$$
n_{2}=c_{0} n+n_{3}
$$

with

$$
\begin{aligned}
n_{3}= & \frac{2}{N_{s}} \sum_{\vec{k}} \int_{-\infty}^{0} \frac{d x}{2 \pi i}\left\{G(\vec{k}, x-i \eta) \frac{d}{d x} \Sigma_{\text {Regular }}(\vec{k}, x-i \eta)\right. \\
& \left.-G(\vec{k}, x+i \eta) \frac{d}{d x} \Sigma_{\text {Regular }}(\vec{k}, x+i \eta)\right\} .
\end{aligned}
$$

Now $n_{3}$ can be integrated by parts safely. We further assume with Luttinger and Ward that $\Sigma_{\text {Regular }}(\vec{k}, z)=\frac{\delta \Phi_{\text {Regular }}}{\delta G(\vec{k}, z)}$ with a suitable functional $\Phi_{\text {Regular }}$. This is the second part of our conjecture alluded to above, and is based upon the idea that the Luttinger-Ward functional $\Phi_{\mathrm{LW}}$ itself can be decomposed into a regular and singular parts. ${ }^{54}$ With this it follows that $n_{3}=0$, as shown in. ${ }^{22,23}$ Thus we find in the EC limit

$$
n=n_{1}+c_{0} n=\left(1-\frac{n}{2}\right) n_{1}
$$

and thus

$$
\frac{n}{1-\frac{n}{2}}=\frac{2}{N_{s}} \sum_{\vec{k}} \Theta[G(\vec{k}, 0)],
$$

where we used Eq. (D4) in obtaining the second line from the first. We emphasize that this renormalized version of the Luttinger-Ward volume theorem, is only applicable in the EC limit as in Eq. (D2), and of course is the same as the FS volume for the ECQL in Eq. (148).

\section{APPENDIX E: THE DIFFERENT LIMITS OF VANISHING SOURCE TERMS}

There are many possible ways to turn off the sources. The generic one used for most calculations is Case 1 below, and we also list some other possibilities for completeness. We may distinguish three possibilities:

Case 1 All source terms

$$
V_{i}^{\sigma_{a}, \sigma_{b} \propto \xi}
$$

where $\xi$ is infinitesimal. In this case we can readily establish that

$$
\mathcal{V}^{(k)}-V^{(k)} \propto\left(\xi^{2}\right) .
$$

The same holds for $\mathcal{V}-V \propto\left(\xi^{2}\right)$ and also $D-\mathcal{D} \propto\left(\xi^{2}\right)$. Hence to linear order in $\xi$ we may ignore the distinction between $\mathcal{V}$ and $V$.

Case 2 Spin diagonal $V$, where

$$
V_{i}^{\uparrow, \downarrow}=V_{i}^{\downarrow, \uparrow}=0 \text {. }
$$

In this case again we see that $V=\mathcal{V}$ since all off-diagonal $\mathcal{G}$ 's vanish identically. This turns out to be not interesting since the $\hat{G}$ 's remain off-diagonal in spin space here because the total source term is not just $V$ (see next case).

Case 3 The spin-polarized case where we choose

$$
V_{i}^{\sigma \bar{\sigma}} \propto \xi
$$

but $\xi$ much smaller than $V_{i}^{\uparrow \uparrow}-V_{i}^{\downarrow}$, the latter being set to zero last. In this case, the symmetry of the underlying ECQL state 
gives us $\mathcal{G}_{\sigma, \bar{\sigma}} \propto \xi$, and $\mathcal{G}_{\sigma, \sigma}-\mathcal{G}_{\bar{\sigma}, \bar{\sigma}}$ is arbitrary. Thus we allow for ferromagnetic polarization in this case. The total source term from Eqs. (B4) and (41) is $V_{t o t}=V+V^{(k)}-\mathcal{V}^{(k)}$. We compute this to first order in $\xi$ and find

$$
\begin{gathered}
\left(V_{i}^{\uparrow \uparrow}\right)_{\text {tot }}=V_{i}^{\uparrow \uparrow}+\mathcal{G}_{\uparrow \downarrow}\left[i^{-}, i\right] \frac{V_{i}^{\uparrow \uparrow}-V_{i}^{\downarrow \downarrow}}{\left(1-\mathcal{G}_{\uparrow \uparrow}\left[i^{-}, i\right]\right)\left(1-\mathcal{G}_{\downarrow \downarrow}\left[i^{-}, i\right]\right)}, \\
\left(V_{i}^{\downarrow \uparrow}\right)_{t o t}=\frac{\left(1-\mathcal{G}_{\downarrow \downarrow}\left[i^{-}, i\right]\right) V_{i}^{\downarrow \uparrow}-\mathcal{G}_{\downarrow \uparrow}\left[i^{-}, i\right]\left(V_{i}^{\uparrow \uparrow}-V_{i}^{\downarrow \downarrow}\right)}{1-\mathcal{G}_{\uparrow \uparrow}\left[i^{-}, i\right]} .
\end{gathered}
$$

The other two components can be read off from the transformation generated by reversing all spins, i.e., $\uparrow \leftrightarrow \downarrow$. From this equation, we learn that the $\hat{G}$ 's will be diagonal in spin space if we adjust

$$
\left(1-\mathcal{G}_{\downarrow \downarrow}\left[i^{-}, i\right]\right) V_{i}^{\downarrow \uparrow}=\mathcal{G}_{\downarrow \uparrow}\left[i^{-}, i\right]\left(V_{i}^{\uparrow \uparrow}-V_{i}^{\downarrow \downarrow}\right)
$$

since the $\hat{G}$ 's respond to the total source term.

\section{APPENDIX F: VARIOUS SUSCEPTIBILITIES AND THEIR RELATIONSHIPS AND SUM RULES}

We need the relationship between the response function $Y$ of the Green's function $\mathcal{G}$ to the external potential $\mathcal{V}$ and the function $\chi$ defined in Eq. (39).

Let us write basic identity

$$
\begin{aligned}
\Upsilon_{\sigma_{c}, \sigma_{d}}^{\sigma_{a} \sigma_{b}}[p, q ; r]= & \frac{\delta \mathcal{G}_{\sigma_{a} \sigma_{b}}[p, q]}{\delta \mathcal{V}_{r}^{\sigma_{c} \sigma_{d}}} /_{V \rightarrow 0} \\
= & \left(1-\frac{n}{2}\right) \chi_{\sigma_{c} \sigma_{d} \sigma_{d} \sigma_{b}}^{\sigma_{0}}[p, q ; r] \\
& -\sigma_{a} \sigma_{b} \Upsilon_{\sigma_{c}, \sigma_{d}}^{\bar{\sigma}_{b} \bar{\sigma}_{a}}[p, p ; r] \hat{G}_{\sigma_{b} \sigma_{b}}[p, q],
\end{aligned}
$$

where we have used the relation $\mathcal{G}[p, q]=\Delta[p] . \hat{G}[p, q]$ and the relations, Eq. (A6). Therefore specializing to the nonvanishing spin configurations we find

$$
\begin{aligned}
& \Upsilon^{(1)}[i, j ; k]-\left(1-\frac{n}{2}\right) \chi^{(1)}[i, j ; k]=-G[i, j] \Upsilon^{(2)}\left[i^{-}, i ; k\right], \\
& \Upsilon^{(2)}[i, j ; k]-\left(1-\frac{n}{2}\right) \chi^{(2)}[i, j ; k]=-G[i, j] \Upsilon^{(1)}\left[i^{-}, i ; k\right], \\
& \Upsilon^{(3)}[i, j ; k]-\left(1-\frac{n}{2}\right) \chi^{(3)}[i, j ; k]=+G[i, j] \Upsilon^{(3)}\left[i^{-}, i ; k\right]
\end{aligned}
$$

and Fourier transforming (with $\mathrm{Y}[Q]=\Sigma_{q} \mathrm{Y}[q, q+Q]$ )

$$
\begin{aligned}
& \Upsilon^{(1)}\left[p_{1}, p_{2}\right]+\Upsilon^{(2)}\left[p_{2}-p_{1}\right] G\left[p_{2}\right]=\left(1-\frac{n}{2}\right) \chi^{(1)}\left[p_{1}, p_{2}\right], \\
& \Upsilon^{(2)}\left[p_{1}, p_{2}\right]+\Upsilon^{(1)}\left[p_{2}-p_{1}\right] G\left[p_{2}\right]=\left(1-\frac{n}{2}\right) \chi^{(2)}\left[p_{1}, p_{2}\right],
\end{aligned}
$$

$$
\Upsilon^{(3)}\left[p_{1}, p_{2}\right]-\Upsilon^{(3)}\left[p_{2}-p_{1}\right] G\left[p_{2}\right]=\left(1-\frac{n}{2}\right) \chi^{(3)}\left[p_{1}, p_{2}\right]
$$

The susceptibilities at finite wave vectors follow by setting $p_{2}=p_{1}+Q$ and summing over $p_{1}$ so that

$$
\begin{aligned}
& \Upsilon^{(1)}[Q]+\frac{n}{2-n} \Upsilon^{(2)}[Q]=\left(1-\frac{n}{2}\right) \chi^{(1)}[Q], \\
& \Upsilon^{(2)}[Q]+\frac{n}{2-n} \Upsilon^{(1)}[Q]=\left(1-\frac{n}{2}\right) \chi^{(2)}[Q], \\
& \Upsilon^{(3)}[Q]-\frac{n}{2-n} \Upsilon^{(3)}[Q]=\left(1-\frac{n}{2}\right) \chi^{(3)}[Q]
\end{aligned}
$$

so that

$$
\begin{gathered}
\chi_{s}[Q]=\frac{1}{\left(1-\frac{n}{2}\right)^{2}} \Upsilon_{s}[Q], \quad \chi_{t}[Q]=\frac{1-n}{\left(1-\frac{n}{2}\right)^{2}} \Upsilon_{t}[Q], \\
\Upsilon_{s}\left[i^{-}, i ; k\right]=\left(1-\frac{n}{2}\right)^{2} \chi_{s}\left[i^{-}, i ; k\right], \\
\Upsilon_{t}\left[i^{-}, i ; k\right]=\frac{\left(1-\frac{n}{2}\right)^{2}}{1-n} \chi_{t}\left[i^{-}, i ; k\right] .
\end{gathered}
$$

In order to gain intuition for these objects, we note that the "physical" (i.e., positive-definite) magnetic and charge susceptibilities (i.e., compressibility ${ }^{26}$ ) at finite wave vectors are given by

$$
\begin{gathered}
\chi_{\text {spin }}[Q]=-2 \mu_{B}^{2} \Upsilon_{t}[Q], \\
\chi_{\text {charge }}[Q]=-\Upsilon_{s}[Q]
\end{gathered}
$$

with the normalization that the corresponding objects for the (noninteracting) Fermi gas are $\chi_{\text {spin }}=2 \mu_{B}^{2} n[0]$ and $\chi_{\text {charge }}$ $=n[0]$, respectively, with $n[0]$ the density of states per spin per site at the chemical potential. Notice that the triplet object $\chi_{t}$ turns up with an explicit factor $1-n$, and so it vanishes near half filling, and conversely the physical magnetic susceptibility is obtained from it by dividing with this factor.

From the definitions $\mathrm{Y}\left[i^{-}, i ; r\right]=\frac{\delta \mathcal{G}\left[i^{-}, i\right]}{\delta \mathcal{V}_{r}} /{ }_{V \rightarrow 0}$ (with suitable spin indices), we can relate the susceptibilities in real space to physically interesting correlations

$$
\begin{aligned}
& \Upsilon^{(1)}\left[i^{-}, i ; j\right]=-\left\langle\left\langle X_{i}^{\uparrow \uparrow} X_{j}^{\uparrow \uparrow}\right\rangle\right\rangle+\left\langle\left\langle X_{i}^{\uparrow \uparrow}\right\rangle\right\rangle\left\langle\left\langle X_{j}^{\uparrow \uparrow}\right\rangle\right\rangle, \\
& \Upsilon^{(2)}\left[i^{-}, i ; j\right]=-\left\langle\left\langle X_{i}^{\uparrow \uparrow} X_{j}^{\downarrow \downarrow}\right\rangle\right\rangle+\left\langle\left\langle X_{i}^{\uparrow \uparrow}\right\rangle\right\rangle\left\langle\left\langle X_{j}^{\downarrow \downarrow}\right\rangle\right\rangle, \\
& \Upsilon^{(3)}\left[i^{-}, i ; j\right]=-\left\langle\left\langle X_{i}^{\uparrow \downarrow} X_{j}^{\downarrow \uparrow}\right\rangle\right\rangle+\left\langle\left\langle X_{i}^{\uparrow \downarrow}\right\rangle\right\rangle\left\langle\left\langle X_{j}^{\downarrow \uparrow}\right\rangle\right\rangle .
\end{aligned}
$$

We next turn off the sources, thus at equal times $\tau_{i}=\tau_{j}$, we may write the correlation functions in terms of the physically meaningful charge and spin correlators 


$$
\Upsilon_{s}\left[i^{-}, i ; j\right]=\frac{1}{2}\left(n^{2}-\left\langle n_{i} n_{j}\right\rangle\right), \quad \Upsilon_{t}\left[i^{-}, i ; j\right]=-\frac{2}{3}\left\langle\vec{S}_{i} \cdot \vec{S}_{j}\right\rangle .
$$

If we also set $i=j$, we to obtain the local-and equal-time susceptibilities

$$
\begin{gathered}
\Upsilon_{\mathrm{loc}}^{(1)}=\frac{n}{2}\left(\frac{n}{2}-1\right) \quad \Upsilon_{\mathrm{loc}}^{(2)}=\left(\frac{n}{2}\right)^{2} \quad \Upsilon_{\mathrm{loc}}^{(3)}=-\frac{n}{2}, \\
\Upsilon_{s, \mathrm{loc}}=-\frac{n}{2}(1-n) \quad \Upsilon_{t, \mathrm{loc}}=-\frac{n}{2} .
\end{gathered}
$$

These are useful for the sum rules that we discuss next.

\section{Susceptibility sum rules}

The local $\chi$ 's follow from Eq. (F5) by summing over $Q$, and these provide us with sum rules for the susceptibilities. We find that both $\chi_{s}$ and $\chi_{t}$ satisfy exactly the same sum rule,

$$
\begin{gathered}
\sum_{Q} \chi_{s}[Q]=\frac{1}{\left(1-\frac{n}{2}\right)^{2}} \sum_{Q} \Upsilon_{s}[Q]=\chi_{\mathrm{loc}}, \\
\sum_{Q} \chi_{t}[Q]=\frac{1-n}{\left(1-\frac{n}{2}\right)^{2}} \sum_{Q} \Upsilon_{t}[Q]=\chi_{\mathrm{loc}}
\end{gathered}
$$

with

$$
\chi_{\mathrm{loc}}=-\frac{n}{2} \frac{(1-n)}{\left(1-\frac{n}{2}\right)^{2}} .
$$

The vanishing with $1-n$ of both the spin and charge $\chi$ 's is an interesting consequence of our construction. The equalized local singlet and triplet sum rules simplify further analysis. The two Y's are the physical susceptibilities, relevant for neutron scattering, NMR, charge response, and other probes. These are naturally distinct from each other, for example, at the insulating limit $n=1$ there is a nonvanishing spin response but no charge response.

\section{APPENDIX G: DETAILED VERTICES}

In this section we present the vertices, where we have dropped the higher-order vertices, i.e., set $\frac{\delta \Gamma}{\delta W} \rightarrow 0$. We first break up the self-energy given in Eq. (43) into convenient smaller terms, and then present the singlet and triplet vertex corrections arising from these six terms.

$$
\begin{aligned}
& \mathcal{S}_{1}[i, j]=-t[i, j]\left[\Delta[j]-\left(1-\frac{n}{2}\right)\right], \quad \mathcal{S}_{2}[i, j]=-t[i, j] \nu[i, j], \\
& \mathcal{S}_{3}[i, j]= t[i, k] \Theta[i, k, j], \quad \mathcal{S}_{4}[i, j]=\delta[i, j] \frac{1}{2} J[i, k] \\
& \times\left\{\mu[i, k] \cdot \Delta[i]-\left(1-\frac{n}{2}\right)\right\},
\end{aligned}
$$

$$
\mathcal{S}_{5}[i, j]=+\delta[i, j] \frac{1}{2} J[i, k] \mu[i, k] \cdot \nu[k, i],
$$

$$
\mathcal{S}_{6}[i, j]=\frac{1}{2} J[i, k] \mu[i, k] \cdot \Theta[k, i, j] .
$$

We pull out the explicit factors of $\lambda=\frac{1}{1-n}$ and present the answers as a series in $\lambda$. The answers are given in real space with the external space-time indices $i, j$, and $m$ and four internal indices (summed over) $a, b, c$, and $k$.

We first write the singlet vertices $\Gamma_{s}[i, j ; m]_{r}=\frac{\delta \mathcal{S}_{r}[i, j]}{\delta V_{m}}$ with $1 \leq r \leq 6$,

$$
\Gamma_{s}[i, j ; m]_{1}=\frac{1}{4}(n-2)^{2} t[i, j] \chi_{s}[j, j, m],
$$

$$
\begin{aligned}
\Gamma_{s}[i, j ; m]_{2}= & \frac{3}{8}(n-2) \lambda t[i, j]\left\{(n-2) \chi_{t}[i, i, j] \chi_{s}[j, j, m]\right. \\
& -2 \Gamma_{t}[a, b, j]\left[\hat{G}[i, a] \chi_{s}[b, i, m]\right. \\
& \left.\left.+\hat{G}[b, i] \chi_{s}[i, a, m]\right]\right\}+\frac{1}{8}(n-2) t[i, j] \\
& \times\left\{-2 \Gamma_{s}[a, b, j]\left[\hat{G}[i, a] \chi_{s}[b, i, m]\right.\right. \\
& \left.+\hat{G}[b, i] \chi_{s}(i, a, m)\right]-(n-2) \chi_{s}[i, i, j] \\
& \left.\times\left[2 \chi_{s}[i, i, m]-\chi_{s}[j, j, m]\right]\right\}+\frac{3}{8}(n \\
& -2)^{2} n \lambda^{2} t[i, j] \chi_{t}[i, i, j] \chi_{s}[i, i, m],
\end{aligned}
$$

$$
\begin{gathered}
\Gamma_{s}[i, j ; m]_{3}=\frac{1}{4} t[i, k]\left[\Gamma_{s}[c, j, i]-3 \Gamma_{t}[c, j, i]\right]\{(n-2) \hat{G}[k, c] \\
\left.\times\left[\chi_{s}[i, i, m]-\chi_{s}[k, k, m]\right]-2 \chi_{s}[k, c, m]\right\}, \\
\Gamma_{s}[i, j ; m]_{4}=-\frac{1}{8}(n-2)^{2} \delta[i, j] J[i, k] \chi_{S}[k, k, m],
\end{gathered}
$$

$$
\begin{aligned}
\Gamma_{s}[i, j ; m]_{5}= & \frac{3}{8}(n-2) \lambda \delta[i, j] J[i, k]\left\{\Gamma_{t}[a, b, i]\right. \\
& \times\left[\hat{G}(k, a) \chi_{s}[b, k, m]+\hat{G}[b, k] \chi_{s}[k, a, m]\right]-(n \\
& \left.-2) \chi_{t}[k, k, i] \chi_{s}[i, i, m]\right\}+\frac{1}{16}(n-2) \delta[i, j] J[i, k] \\
& \times\left\{2 \Gamma _ { s } [ a , b , i ] \left[\hat{G}[k, a] \chi_{s}[b, k, m]\right.\right. \\
& \left.\left.+\hat{G}[b, k] \chi_{s}[k, a, m]\right]\right\}-(n-2)^{2} \delta[i, j] J[i, k] \\
& \times\left\{\chi_{s}[k, k, i]\left[2 \chi_{s}[i, i, m]-3 \chi_{s}[k, k, m]\right]\right\} \\
& -\frac{3}{16}(n-2)^{2}(2 n-1) \lambda^{2} \delta[i, j] J[i, k] \chi_{t}[k, k, i] \\
& \times \chi_{s}[k, k, m],
\end{aligned}
$$


$\Gamma_{s}[i, j ; m]_{6}=\frac{1}{4} J[i, k] \chi_{s}[i, c, m]\left(\Gamma_{s}[c, j, k]-3 \Gamma_{t}[c, j, k]\right)$.

(G2)

We next note the triplet vertices

$$
\Gamma_{t}[i, j ; m]_{1}=-\frac{1}{4}(n-2)^{2} \lambda t[i, j] \chi_{t}[j, j, m],
$$

$$
\begin{aligned}
\Gamma_{t}[i, j ; m]_{2}= & {\left[\frac{1}{8}(n-2) \lambda_{t}[i, j]\right]\left\{2 \hat { G } [ i , a ] \chi _ { t } [ b , i , m ] \left[\Gamma_{s}[a, b, j]\right.\right.} \\
& \left.-(n+1) \Gamma_{t}[a, b, j]\right]+2 \hat{G}[b, i] \chi_{t}[i, a, m]\left[\Gamma_{s}[a, b, j]\right. \\
& \left.-(n-3) \Gamma_{t}[a, b, j]\right]-(n-2)\left[\chi_{s}[i, i, j] \chi_{t}[j, j, m]\right. \\
& \left.\left.+2 \chi_{t}[i, i, j] \chi_{t}[i, i, m]\right]\right\}-\frac{1}{8}(n-2)^{2} \lambda^{2} t[i, j] \\
& \times\left\{n \chi_{s}[i, i, j] \chi_{t}[i, i, m]-\chi_{t}[i, i, j] \chi_{t}[j, j, m]\right\},
\end{aligned}
$$$$
\Gamma_{t}[i, j ; m]_{3}=-\frac{1}{4}(n-2) \lambda \hat{G}[k, c] t[i, k]\left(\chi_{t}[i, i, m]-\chi_{t}[k, k, m]\right)
$$$$
\times\left(\Gamma_{s}[c, j, i]+\Gamma_{t}[c, j, i]\right)-\frac{1}{2} t[i, k] \chi_{t}[k, c, m]
$$$$
\times\left(\Gamma_{s}[c, j, i]+\Gamma_{t}[c, j, i]\right),
$$

$$
\Gamma_{t}[i, j ; m]_{4}=\frac{1}{8}(n-2)^{2} \lambda \delta[i, j] J[i, k] \chi_{t}[k, k, m]
$$

$$
\begin{aligned}
\Gamma_{t}[i, j ; m]_{5}= & {\left[\frac{1}{8}(n-2) \lambda \delta[i, j] J[i, k]\right] } \\
& \times\left\{\hat { G } [ k , a ] \chi _ { t } [ b , k , m ] \left[(n+1) \Gamma_{t}[a, b, i]\right.\right. \\
& \left.-\Gamma_{s}[a, b, i]\right]+(n-2) \chi_{s}[k, k, i] \chi_{t}[i, i, m] \\
& \left.-\hat{G}[b, k] \chi_{t}[k, a, m]\left[\Gamma_{s}[a, b, i]-(n-3) \Gamma_{t}[a, b, i]\right]\right\} \\
& -\frac{1}{16}(n-2)^{2} \lambda^{2} \delta[i, j] J[i, k]\left\{\chi_{t}[k, k, m][(1\right. \\
& \left.-2 n) \chi_{s}[k, k, i]+(2 n+1) \chi_{t}[k, k, i]\right] \\
& \left.-2 \chi_{t}[k, k, i] \chi_{t}[i, i, m]\right\},
\end{aligned}
$$

$$
\begin{aligned}
\Gamma_{t}[i, j ; m]_{6}= & \frac{1}{4} J[i, k] \chi_{t}[i, c, m]\left(\Gamma_{s}[c, j, k]+\Gamma_{t}[c, j, k]\right) \\
& -\frac{1}{2}(n-2) \lambda \hat{G}[i, c] J[i, k] \Gamma_{t}[c, j, k]\left(\chi_{t}[i, i, m]\right. \\
& \left.-\chi_{t}[k, k, m]\right)
\end{aligned}
$$

\section{APPENDIX H: FOURIER-TRANSFORM CONVENTION}

Our convention for various Fourier transforms is summarized here.

$$
\begin{aligned}
& p=\left(\vec{p}, \omega_{p}\right), \quad r=(\vec{r}, \tau), \quad \omega_{p}=2 \pi\left(p+\frac{1}{2}\right) k_{B} T, \\
& p r=\vec{p} \cdot \vec{r}-\omega_{p} \tau, \\
& \sum_{k}=\frac{1}{N_{s}} k_{B} T \sum_{\omega_{n}} \sum_{\vec{k}} \\
& G[a, b]=\sum_{k} e^{i k(a-b)} G[k], \\
& \chi(a, b ; c)=\sum_{p_{1}, p_{2}} \chi\left[p_{1}, p_{2}\right] e^{i\left[p_{1}(a-c)+p_{2}(c-b)\right]}, \\
& \chi\left[p_{1}, p_{2}\right]=\frac{1}{N_{s}} \sum_{\vec{r}_{a}, \vec{r}_{b}, \vec{r}_{c}} \int_{0}^{\beta} \int_{0}^{\beta} d\left(\tau_{a}-\tau_{c}\right) d\left(\tau_{c}\right. \\
& \left.-\tau_{b}\right) e^{-i\left[p_{1}(a-c)+p_{2}(c-b)\right]} \chi[a, b ; c], \\
& \Lambda[a, b ; c]=\sum_{p_{1}, p_{2}} \Lambda\left[p_{1}, p_{2}\right] e^{i\left[p_{1}(a-c)+p_{2}(c-b)\right]}, \\
& \nu[a, b]=\sum_{Q} \nu[Q] e^{-i[Q(a-b)]}, \\
& \chi\left[p_{1}, p_{2}\right]=G\left[p_{1}\right] \Gamma\left[p_{1}, p_{2}\right] G\left[p_{2}\right], \\
& t[i, j]=-\sum_{p} \varepsilon_{p} e^{i p\left(r_{i}-r_{j}\right)}, \\
& J[i, j]=\sum_{p} J_{p} e^{i p\left(r_{i}-r_{j}\right)}, \\
& \chi[Q]=\sum_{p} \chi[p, p+Q], \\
& \Upsilon[Q]=\sum_{p} \Upsilon[p, p+Q] .
\end{aligned}
$$


${ }^{1}$ T. V. Ramakrishnan and B. S. Shastry, in Theoretical and Experimental Aspects of Valence Fluctuations and Heavy Fermions, International Conference on Valence Fluctuations, edited by L. C. Gupta and S. K. Malik (Plenum, New York, 1987), http://physics.ucsc.edu/ sriram/papers/paper_33.pdf.

${ }^{2} \mathrm{An}$ example of such a strong-coupling liquid is the quantum Heisenberg paramagnet at a sufficiently high temperature. It has translation invariance and rotation invariance intact but has no simple adiabatic relationship to a free gas with the same symmetries. The ECQL takes such a spin liquid and further introduces mobile charges into it.

${ }^{3}$ W. Metzner and D. Vollhardt, Phys. Rev. Lett. 62, 324 (1989).

${ }^{4}$ A. Georges, G. Kotliar, W. Krauth, and M. J. Rozenberg, Rev. Mod. Phys. 68, 13 (1996).

${ }^{5}$ P. A. Lee, N. Nagaosa, and X. G. Wen, Rev. Mod. Phys. 78, 17 (2006).

${ }^{6}$ E. Arrigoni, M. Aichhorn, M. Daghofer, and W. Hanke, New J. Phys. 11, 055066 (2009); F. C. Zhang and T. M. Rice, Phys. Rev. B 37, 3759 (1988); B. S. Shastry, Phys. Rev. Lett. 63, 1288 (1989).

${ }^{7}$ A. M. Shvaika, Phys. Rev. B 62, 2358 (2000); Yu. A. Izyumov, B. M. Letfulov, E. V. Shipitsyn, M. Bartkowiak, and K. A. Chao, ibid. 46, 15697 (1992).

${ }^{8}$ P. W. Anderson, Science 235, 1196 (1987); The Theory of Superconductivity (Princeton University Press, Princeton, NJ, 1997); arXiv:0709.0656 (unpublished).

${ }^{9}$ S. E. Barnes, J. Phys. F: Met Phys. 6, 1375 (1976); N. Read and D. Newns, J. Phys. C 16, 3273 (1983); P. Coleman, Phys. Rev. B 29, 3035 (1984); G. B. Kotliar and A. E. Ruckenstein, Phys. Rev. Lett. 57, 1362 (1986).

${ }^{10}$ M. C. Gutzwiller, Phys. Rev. Lett. 10, 159 (1963); W. F. Brinkman and T. M. Rice, Phys. Rev. B 2, 4302 (1970).

${ }^{11}$ G. C. Wick, Phys. Rev. 80, 268 (1950).

${ }^{12}$ F. J. Dyson, Phys. Rev. 75, 486 (1949).

${ }^{13}$ J. Schwinger, Phys. Rev 74, 1439 (1948); Proc. Natl. Acad. Sci. U.S.A. 37, 452 (1951); P. C. Martin and J. Schwinger, Phys. Rev. 115, 1342 (1959).

${ }^{14}$ G. Baym and L. P. Kadanoff, Phys. Rev. 124, 287 (1961); L. P. Kadanoff and G. Baym, Quantum Statistical Mechanics: Green's Function Methods in Equilibrium and Nonequilibrium Problems (Benjamin, NY, 1962); G. Baym, Phys. Rev. 127, 1391 (1962).

${ }^{15}$ A. K. Rajagopal, Nucl. Phys. 57, 435 (1964); A. K. Rajagopal, H. Brooks, and N. Ranganathan, Nuovo Cimento, Suppl. 5, 807 (1967).

${ }^{16}$ In common with standard many-body theory (Refs. 23 and 26), the Green's function is expressed in terms of a self-energy, the self-energy in terms of a vertex function, and the vertex function satisfies integral equations involving various susceptibilities and also higher-order vertices.

${ }^{17}$ R. J. Rivers, Path Integral Methods in Quantum Field Theory (Cambridge University Press, Cambridge, U.K., 1987).

${ }^{18}$ In standard perturbation theory, e.g., as discussed in Refs. 22, 23, and 26, an expansion in bare Green's functions is resummed to give the skeleton graph expansion for the self-energy $\Sigma$, or compact diagrams for the grand potential $\Omega$, where the internal lines are all the full Green's function. The Schwinger source technique quite naturally generates a series that is completely analogous to this skeleton graph expansion and bypasses the first stage of bare lines. In this sense, the lack of Wick's theorem for the Hubbard operators that prevents a regular Feynman-type analysis, is overcome by employing the Schwinger approach.

${ }^{19}$ It is important to verify that the self-energy and vertices are well behaved at high frequencies $\omega$ (i.e., have the form $\sim c_{1}+\frac{c_{2}}{\omega}$ ), and possess proper spectral representations. When these conditions are not satisfied, as with equations formally following from those of $\mathcal{G}$ without the removal of the factor of $\Delta$ as in Eq. (31), we denote the resulting equations as ill formed.

${ }^{20}$ Our choice of a left-handed decomposition of $\Delta[i]$ in Eq. (31) has the consequence that in the following theory, we will end up with objects, such as vertices and irreducible interactions that are not symmetric under exchanging the incoming and outgoing momenta, i.e., we will lack manifest time-reversal invariance. One work, around is to take the resulting equations, and to symmetrize them explicitly as need arises, as, e.g., in Eq. (134). It is also possible to work with the asymmetric objects, and unsurprisingly lead to the same conclusions for instabilities, etc. More directly, we can define a symmetrically factored $\mathcal{G}[i, f]=\sqrt{\Delta[i]} \cdot G_{s}[i, f] \cdot \sqrt{\Delta[f]} \quad$ so that $G_{s}^{-1}[i, f]$ $=\sqrt{\Delta[f]} \cdot \hat{G}^{-1}[i, f] \cdot \sqrt{\Delta^{-1}[i]}$. From this it follows that the vertices $\Lambda^{a}$ obtained from $G_{s}$ by differentiating w.r.t. the sources are related to the $\Gamma$ 's used here straightforwardly. Taking spin diagonal sources we find via $\Lambda_{\sigma^{\prime} \sigma^{\prime}}^{\sigma \sigma}[i, f ; m]=\Gamma_{\sigma^{\prime} \sigma^{\prime}}^{\sigma \sigma}[i, f ; m]$ $-\left.\frac{1}{2} \hat{G}_{\sigma \sigma}^{-1}[i, f] \frac{\delta \mu_{\sigma \sigma}[i, f]}{\delta V_{m}^{\sigma^{\prime} \sigma^{\prime}}}\right|_{V \rightarrow 0}$. The added terms do not lead to any pathologies at high frequency. The added terms, explicitly available from Eq. (74), vanish sufficiently rapidly at high frequency to damp out the growing terms from $\hat{G}^{-1}[i, f]$. We can then convert the asymmetric $\Gamma$ vertices to the symmetric vertices $\Lambda$ using this prescription.

${ }^{21}$ This represents the dynamical inverse and should not to be confused with a matrix inverse.

22 J. M. Luttinger and J. C. Ward, Phys. Rev 118, 1417 (1960); J. M. Luttinger, Phys. Rev. 119, 1153 (1960); 121, 942 (1961).

${ }^{23}$ A. A. Abrikosov, L. Gorkov, and I. Dzyaloshinski, Methods of Quantum Field Theory in Statistical Physics (Prentice-Hall, Englewood Cliffs, NJ, 1963).

${ }^{24}$ G. D. Mahan, Many Particle Physics, 2nd ed. (Plenum, New York, 1991).

${ }^{25}$ M. Potthoff, M. Aichhorn, and C. Dahnken, Phys. Rev. Lett. 91, 206402 (2003).

${ }^{26}$ P. Nozières, Theory of Interacting Fermi Systems (W. A. Benjamin, Amsterdam, 1964).

${ }^{27}$ J. C. Ward, Phys. Rev. 78, 182 (1950).

${ }^{28}$ Y. Takahashi, Nuovo Cimento 10, 370 (1957).Takahashi's relativistic version of the conservation law is easily generalized as here for a discrete hopping problem with arbitrary band structure.

${ }^{29}$ S. K. Ma, M. Beal-Monod, and D. Fredkin, Phys. Rev. 174, 227 (1968).

${ }^{30}$ On the other hand, for the Hubbard model, it is easy to show that the triplet vertices satisfy finite versions of the Ward identity analogous to Eqs. (90) and (91), since the spin density commutes with the interaction terms.

${ }^{31}$ B. S. Shastry, Rep. Prog. Phys. 72, 016501 (2009).

${ }^{32}$ B. S. Shastry et al. (unpublished).

${ }^{33}$ J. Hubbard, Proc. R. Soc. London, Ser. A 276, 238 (1963).

${ }^{34}$ J. Hubbard, Proc. R. Soc. London, Ser. A 277, 237 (1964); 281, 401 (1964).

${ }^{35}$ C. Herring, Magnetism, edited by G. T. Rado and H. Suhl (Academic, NY 1966), Vol. IV, p. 229. 
${ }^{36}$ The Hubbard-I solution (Ref. 33) of the finite- $U$ version has been criticized in literature (Ref. 35), for failing to reproduce the Luttinger volume theorem even in the $U \rightarrow 0$ limit. We see here that while it remains incorrect for all finite values of $U$, it does give the correct renormalized volume at $U \rightarrow \infty$.

${ }^{37}$ P. W. Anderson and W. F. Brinkman, Phys. Rev. Lett. 30, 1108 (1973).

${ }^{38}$ A. J. Leggett, Rev. Mod. Phys. 47, 331 (1975). Leggett's recommendation of using a pseudopotential (page 344 footnote 6) is valuable in our context.

${ }^{39}$ A. J. Leggett, Quantum Liquids (Oxford University Press, Oxford, 2006), see Sec. 6.2 on anisotropic Cooper pairing. Leggett's discussion of the neglect of the Fock terms in favor of the Hartree terms on pages $210-211$ is germane to our similar neglect of the Fock terms in the NLHA.

${ }^{40}$ The extended $s$-wave channel is also excluded by force in our projections and so this treatment does not give a fair chance to that specific order.

${ }^{41}$ S. Nakajima, Prog. Theor. Phys. 50, 1101 (1973).

${ }^{42}$ G. Baskaran, Z. Zhou, and P. W. Anderson, Solid State Commun. 63, 973 (1987).

${ }^{43}$ G. Kotliar, Phys. Rev. B 37, 3664 (1988).

${ }^{44}$ P. W. Anderson, Basic Notions in Condensed Matter (Benjamin/ Cummings, New York, 1984).

${ }^{45}$ High-temperature series expansion studies in W. O. Putikka, M. U. Luccini, and R. R. P. Singh, Phys. Rev. Lett. 81, 2966 (1998) suggest different factors $\xi^{*}>1$, that depend upon the object that is monitored; exact diagonalizations computing the FS volume in J. Kokalj and P. Prelovsek, Phys. Rev. B 75, 045111 (2007), suggest a factor $\xi^{*}$ between 1.2 and 1.8 for 18 electrons on a 20 site cluster, for different $J / t$ 's, where Eq. (148) gives $\xi^{*} \sim 1.8$.

${ }^{46}$ B. S. Shastry, B. I. Shraiman, and R. R. P. Singh, Phys. Rev. Lett. 70, 2004 (1993).

${ }^{47}$ R. Zeyher and M. L. Kulic, Phys. Rev. B 53, 2850 (1996); 54,
8985 (1996); A. Greco and R. Zeyher, Europhys. Lett. 35, 115 (1996).

${ }^{48}$ At the time of completing the mansuscript, we noticed papers on the $t$ - $J$ model, Ref. 47, with some initial overlap with our method. We differ in much of the details and the conclusions. These authors consider only density (but not spin)-dependent sources, and thus do not obtain the analogs of most of our main results, e.g., the exact Schwinger Dyson equation, Eq. (54). They further make what seem to be uncontrolled approximations, leading to results that are in disagreement with ours. For instance, they find an effective band that vanishes as $(1-n)$ near half filling, in contrast to our reduction in the bandwidth by spin and density correlations.

${ }^{49}$ Prof. P. Phillips has brought to my attention a recent preprint: S. Chakraborty, S. Hong, and P. Phillips, arXiv:0909.3069 (unpublished), where charge $2 e$ objects arise dynamically in the Hubbard model.

${ }^{50}$ Its functional derivatives w.r.t. the sources also seems negligible at first sight but we plan to check this more carefully later. For now it must be taken as an assumption for the vertex that is certainly consistent with the Ward identity.

${ }^{51}$ This analysis parallels that of Sec. 6.1 for the Bethe Salpeter equations for particle-hole multiple scattering (Ref. 26).

${ }^{52}$ There is another way to group particles and holes leading to a second particle-hole channel familiar in parquet theory but we will choose this present one since it is most relevant.

${ }^{53}$ The vertices obtained from this atomic Green's function and the "untreated" self-energy where the transformation, Eq. (31), has not been done, are easily seen to contain linear terms at high frequency and hence are examples of a "sick" theory.

${ }^{54}$ We have preliminary results showing how the singular part of the self-energy and the Luttinger-Ward functional arise from a partial summation of an infinite set of diagrams in perturbation theory of the Hubbard model. 